A New Multicomponent Multicatalyst Reaction (MC) ${ }^{2}$ R: Chemoselective Cycloaddition and Latent Catalyst Activation for the Synthesis of Fully Substituted 1,2,3-Triazoles

Kosuke Yamamoto, Theodora Bruun, Jung Yun Kim, Lei Zhang and Mark Lautens* Department of Chemistry, University of Toronto

80 St. George St., Toronto, Ontario, M5S 3H6, Canada

E-mail: mlautens@chem.utoronto.ca

Supporting Information

Table of Contents

General Experimental Procedures ....................................................... 2

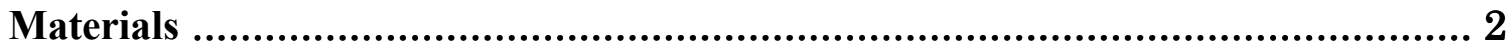

Instrumentation............................................................................. 2

Competition studies of Pd-catalyzed undesired reaction ................................... 3

Optimization of the "all-in-one" (MC) ${ }^{2} \mathrm{R}$.................................................... 3

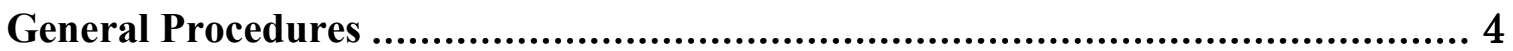

General Procedure A: Synthesis of Iodoalkynes ..................................................4

General Procedure B: Synthesis of Azides.........................................................

General Procedure C: All-in-One Cu/Pd-Catalyzed Triazole Synthesis........................4

General Procedure D: Cyclization of Products ..............................................5

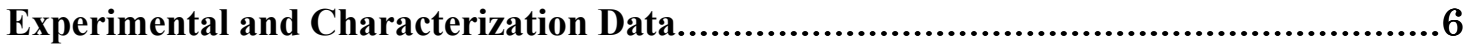

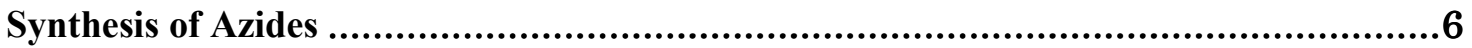

Synthesis of Iodoalkynes ...............................................................................

Synthesis of Terminal alkyne ....................................................................... 11

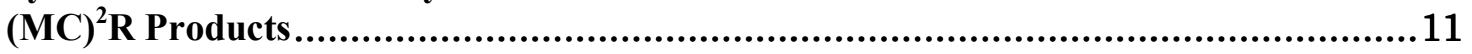

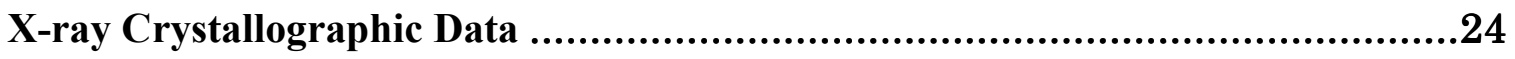

NMR Spectroscopic Data ...........................................................28 


\section{General Experimental Procedures}

Unless otherwise stated, all reactions were performed in dry solvents under an inert atmosphere in oven-dried $\left(120^{\circ} \mathrm{C}\right)$ flasks equipped with a magnetic stir bar. Dry solvents were transferred via syringe. Organic solutions were concentrated by rotary evaporation at $25-40{ }^{\circ} \mathrm{C}$ under reduced pressure. Analytical thin layer chromatography (TLC) was performed on pre-coated EMD Silica gel $60 \mathrm{~F}_{254}$ plates $(0.2 \mathrm{~mm}, 60 \AA$ pore size). Visualization was done with $254 \mathrm{~nm}$ UV light source and generally by immersion in potassium permanganate $\left(\mathrm{KMnO}_{4}\right)$, subsequently followed by heating with a heat gun. Flash chromatography was performed with Silicycle ${ }^{\mathrm{TM}}$ Ultra-Pure $230-400$ mesh silica gel.

\section{Materials}

All reagents were purchased from commercial sources and used as received: catalysts and ligands were purchased from Strem or Sigma Aldrich. All starting materials were obtained from Aldrich, Combi Blocks and VWR. Tetrahydrofuran and dioxane were distilled over $\mathrm{Na}$ /benzophenone prior to use. Toluene was distilled over $\mathrm{CaH}_{2}$ prior to use.

\section{Instrumentation}

Unless otherwise stated, proton nuclear magnetic resonance spectra $\left({ }^{1} \mathrm{H}\right.$ NMR) and carbon nuclear magnetic resonance spectra $\left({ }^{13} \mathrm{C}\right.$ NMR) were recorded at $23{ }^{\circ} \mathrm{C}$ with a Bruker Avance III $400 \mathrm{MHz}$, Varian Mercury $400 \mathrm{MHz}$, Varian Mercury $300 \mathrm{MHz}$, or Agilent DDR 500 spectrometer. Chemical shifts are reported in parts per million (ppm) and referenced to the residual protium relative to TMS $\left(\mathrm{CDCl}_{3}: \delta 7.26\right.$ for ${ }^{1} \mathrm{H}$ NMR and $\delta$ 77.16 for ${ }^{13} \mathrm{C}$ NMR). Spectral data are represented as follows: chemical shift $(\delta)$, multiplicity $(\mathrm{s}=$ singlet, $\mathrm{d}=$ doublet, $\mathrm{t}=$ triplet, $\mathrm{q}=$ quartet, $\mathrm{qn}=$ quintuplet, $\mathrm{sx}=$ sextet, $\mathrm{dd}=$ doublet of doublets, $\mathrm{m}=$ multiplet, $\mathrm{br}=$ broad), coupling constant $(J, \mathrm{~Hz})$, integration. Infrared (IR) spectra were obtained using a Shimadzu FTIR 8400S spectrometry as a film on a $\mathrm{NaCl}$ plate or a Perkin-Elmer Spectrum 100 instrument equipped with a single-bounce diamond / ZnSe ATR accessory. Data is presented as frequency of absorption $\left(\mathrm{cm}^{-1}\right)$. All melting points were determined using a Fisher-Johns melting point apparatus. High resolution mass spectra were obtained from SI2 Micromass $70 \mathrm{~S}-250$ mass spectrometer (EI) or an AB SCIEX QSTAR ${ }^{\circledR}$ mass spectrometer (ESI) or a $\mathrm{JEOL}^{\circledR}$ AccuTOF medel JMS-T1000LC mass spectrometer equipped with an IONICS ${ }^{\circledR}$ Direct Analysis in real Time (DART) ion source. 


\section{Competition studies of Pd-catalyzed undesired reactions}

\section{Table S1. Stability of iodoalkyne 2a in the presence of Pd catalyst}

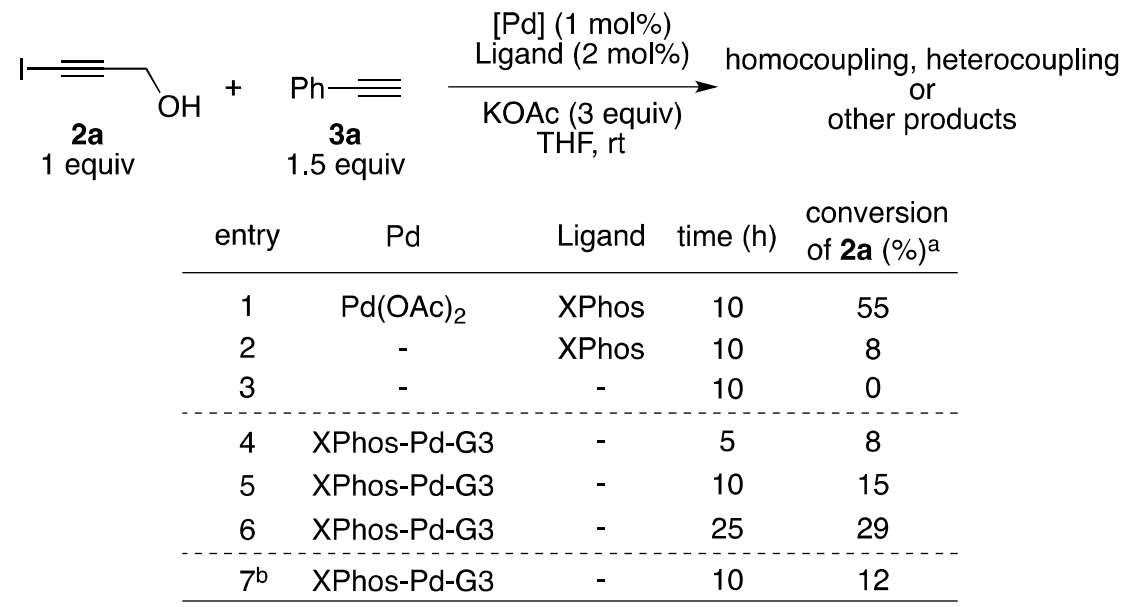

${ }^{a}$ Determined by ${ }^{1}$ H NMR spectroscopy. ${ }^{b}$ Reaction was performed without KOAc.

When the iodoalkyne 2a was subjected to $\mathrm{Pd}(\mathrm{OAc})_{2} / \mathrm{XPhos}(1 \mathrm{~mol} \% \mathrm{Pd}, \mathrm{Pd} / \mathrm{L}=1: 2)$ in the presence of excess amount of phenylacetylene 3a and KOAc at room temperature, we observed 55\% conversion of 2a by ${ }^{1} \mathrm{H}$ NMR analysis after 10 hours (entry 1). On the other hand, using XPhos-Pd-G3 instead of $\mathrm{Pd}(\mathrm{OAc})_{2} / \mathrm{XPhos}$ gave $15 \%$ conversion of 2a after the same reaction time (entry 5). When the reaction was performed using XPhos-PdG3 without KOAc, we also observed $12 \%$ conversion of $\mathbf{2 a}$ (entry 7). These results indicated that KOAc might not generate $\operatorname{Pd}(0)$ species at room temperature, and the conversion of 2a might arise from a spontaneous decomposition of XPhos-Pd-G3.

\section{Optimization of the "all-in-one" (MC) $)^{2} \mathrm{R}$}

Table S2. Effect of the amount of catalysts loading and terminal alkyne ${ }^{\mathrm{a}}$
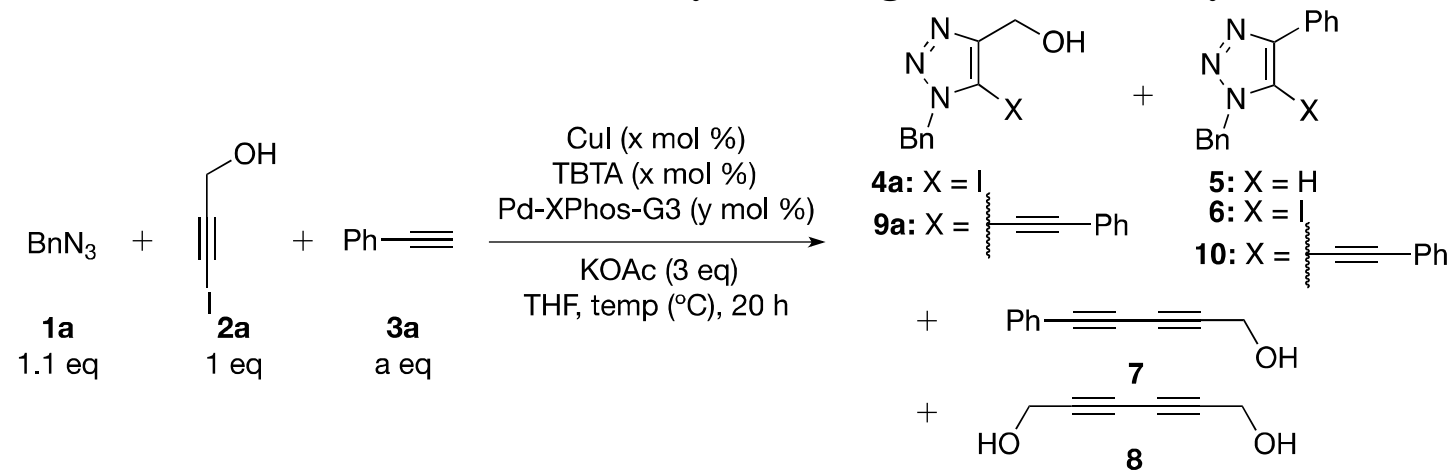


\begin{tabular}{|c|c|c|c|c|c|c|c|c|c|c|c|}
\hline \multirow{2}{*}{ entry } & \multirow{2}{*}{ a } & \multirow{2}{*}{$x$} & \multirow{2}{*}{$\mathrm{y}$} & \multirow{2}{*}{ temp } & \multicolumn{7}{|c|}{ yield $^{\mathrm{b}}(\%)$} \\
\hline & & & & & $9 a$ & $4 a$ & 5 & 6 & 7 & 8 & 10 \\
\hline 1 & 1.5 & 10 & 2 & 85 & 30 & 8 & 14 & 0 & 13 & - & 3 \\
\hline 2 & 2.5 & 10 & 2 & 85 & 8 & 46 & 9 & 0 & 6 & - & - \\
\hline 3 & 1.5 & 10 & 2 & rt $(4 \mathrm{~h})$ then 85 & 67 & 3 & 6 & 0 & 3 & - & 5 \\
\hline 4 & 1.5 & 5 & 2 & rt $(4 \mathrm{~h})$ then 85 & 66 & 2 & 5 & 0 & 2 & trace & 6 \\
\hline 5 & 1.5 & 5 & 1 & rt $(4 \mathrm{~h})$ then 85 & $71(73)$ & 2 & 4 & 0 & 1 & trace & 6 \\
\hline $6^{c}$ & 1.5 & 5 & 1 & rt (4h) & 1 & 80 & 5 & 12 & 1 & 6 & 0 \\
\hline 7 & 1.5 & 2 & 2 & rt $(8 \mathrm{~h})$ then 85 & 63 & 0 & 10 & 0 & 3 & - & 5 \\
\hline
\end{tabular}

a Typical reaction procedure: in a microwave vial under an argon atmosphere, $\mathrm{CuI}$, TBTA, XPhos-Pd-G3, and KOAc were added, followed by the addition of iodoalkyne $(0.2 \mathrm{mmol})$ and benzyl azide (1.1 equiv). The mixture was dissolved in THF $(0.1 \mathrm{M})$ and phenylacetylene was subsequently added. The reaction was stirred at indicated temperature for indicated time. ${ }^{b}$ Yields were determined by ${ }^{1} \mathrm{H}$ NMR spectroscopy of the crude mixture using 1,3,5-trimethoxybenzene as the internal standard. The yield in parentheses is an isolated yield. ${ }^{c}$ The reaction was stopped after stirring at room temperature for 4 hours.

\section{General Procedures}

\section{General Procedure A: Synthesis of Iodoalkynes}

This procedure was adapted from the literature. ${ }^{1}$ To a solution of terminal alkyne (1.0 equiv) stirring in $\mathrm{MeOH}$, an aqueous solution of $\mathrm{KOH}\left(2.5\right.$ equiv) was added at $0^{\circ} \mathrm{C}$. After 10 minutes, $\mathrm{I}_{2}$ (1.1 equiv) was added in one portion and the solution was warmed to room temperature. After stirring at room temperature (3-16 hours) the solution was diluted with $\mathrm{H}_{2} \mathrm{O}$ and extracted with $\mathrm{Et}_{2} \mathrm{O}$. The combined organic layers were washed with aqueous $\mathrm{Na}_{2} \mathrm{~S}_{2} \mathrm{O}_{3}$ solution, brine, and then dried $\left(\mathrm{Na}_{2} \mathrm{SO}_{4}\right)$, filtered and concentrated in vacuo to afford the iodoalkyne without further purification.

\section{General Procedure B: Synthesis of Azides}

This procedure was adapted from the literature. ${ }^{2}$ In a round bottom flask, alkyl bromide ( 1 equiv) was dissolved in DMSO and $\mathrm{NaN}_{3}$ (2 equiv) was added slowly. The reaction was stirred at room temperature and monitored by TLC. Upon conversion of starting materials the reaction was poured into ice water and extracted with $\mathrm{Et}_{2} \mathrm{O}$. The combined organic extracts were washed with water, brine, dried $\left(\mathrm{Na}_{2} \mathrm{SO}_{4}\right)$, filtered, and concentrated in vacuo to afford the azide without further purification.

\section{General Procedure C: All-in-One Cu/Pd-Catalyzed Triazole Synthesis}

To an oven-dried microwave vial under an argon atmosphere equipped with a stir bar $\mathrm{CuI}$ (0.05 equiv), Tris[(1-benzyl-1H-1,2,3-triazol-4-yl)methyl]amine (TBTA) (0.05 equiv), XPhos-Pd-G3 (0.01 equiv), and KOAc ( 3 equiv) were added, followed by the addition of iodoalkyne ( 1 equiv), and azide (1.1 equiv). The mixture was dissolved in THF (0.1 M) and terminal acetylene ( 1.5 equiv) was subsequently added. The reaction was stirred at

\footnotetext{
${ }^{1}$ Denmark, S. E.; Yang, S.-M. J. Am. Chem. Soc. 2002, 124, 2102-2103.

2 Alvarez, S. G.; Alvarez, M. T. Synthesis 1997, 413-414.
} 
room temperature. After TLC analysis showed complete starting material conversion (1$10 \mathrm{~h}$ ) the vial was sealed and stirred at $85{ }^{\circ} \mathrm{C}$ for $20 \mathrm{~h}$. The crude reaction mixture was then cooled to room temperature, filtered through silica pad with ethyl acetate, and the filtrate was concentrated under reduced pressure. Purification by flash column chromatography afforded the desired product.

\section{General Procedure D: Cyclization of Products}

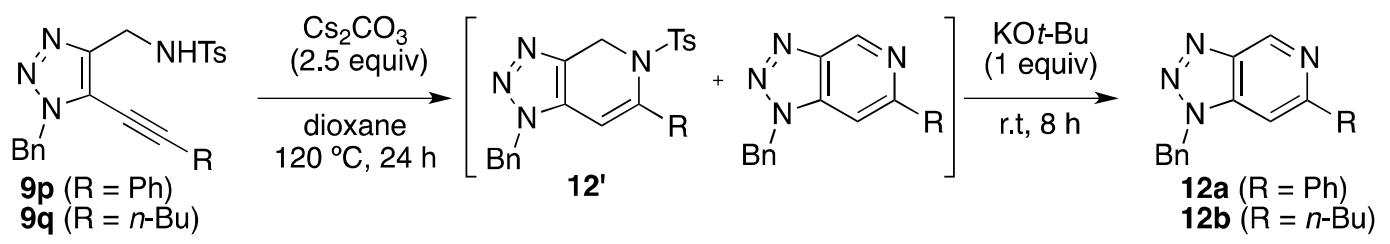

A 2-dram vial with a stir bar was charged with triazole 9p, q $(0.2 \mathrm{mmol}, 1$ equiv), $\mathrm{Cs}_{2} \mathrm{CO}_{3}$ (2.5 equiv), and dioxane $(1 \mathrm{M})$. The vial was sealed with a teflon coated cap and stirred at $120{ }^{\circ} \mathrm{C}$ for $24 \mathrm{~h}$. The mixture was cooled to room temperature and $\mathrm{KO} t$-Bu $(1.0$ equiv) was added followed by stirring at room temperature for an additional $8 \mathrm{~h}$. The mixture was diluted with EtOAc, filtered through a pad of silica gel, and concentrated. The crude mixture was purified by silica gel column chromatography to afford the triazolopyridines 12a, b. 


\section{Experimental and Characterization Data}

\section{Synthesis of Azides}

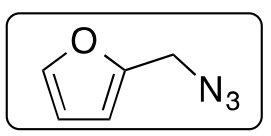

\section{2-(azidomethyl)furan (1h)}

This procedure was adapted from the literature. ${ }^{3}$ To a solution of furfuryl alcohol (491 $\mathrm{mg}, 5 \mathrm{mmol}, 1$ equiv) and diphenylphosphoryl azide ( $1.65 \mathrm{~g}, 1.30 \mathrm{ml}, 6 \mathrm{mmol}, 1.2$ equiv) in toluene $(17 \mathrm{~mL})$ was added DBU $\left(913 \mathrm{mg}, 0.9 \mathrm{ml}, 6 \mathrm{mmol}, 1.2\right.$ equiv) at $0{ }^{\circ} \mathrm{C}$ under an argon atmosphere. The reaction mixture was stirred for $12 \mathrm{~h}$ at room temperature. The mixture was washed with $\mathrm{H}_{2} \mathrm{O}, 1 \mathrm{M}$ aqueous $\mathrm{HCl}$, brine, dried over $\mathrm{Na}_{2} \mathrm{SO}_{4}$, filtered and concentrated under reduced pressure to afford a light yellow oil as a crude mixture. The crude mixture was purified by flash column chromatography over silica gel ( $n$ hexane/AcOEt 95:5) afforded the product as light yellow oil in 31\% yield (188 $\mathrm{mg})$. The characterization data was consistent to that reported in the literature. ${ }^{3}{ }^{1} \mathbf{H}$ NMR (400 $\left.\mathrm{MHz}, C D C l_{3}\right): \delta 7.43(\mathrm{dd}, J=1.9,0.9 \mathrm{~Hz}, 1 \mathrm{H}), 6.42-6.30(\mathrm{~m}, 2 \mathrm{H}), 4.29(\mathrm{~s}, 2 \mathrm{H}) ;{ }^{13} \mathbf{C}$ NMR (101 MHz, $\left.C D C l_{3}\right): \delta 149.2,143.5,110.6,109.5,47.1$.

$$
\mathrm{C}^{\mathrm{S}} \mathrm{N}_{3}
$$

\section{2-(azidomethyl)thiophene (1i)}

To a solution of 2-(thiophene)methanol (554 $\mathrm{mg}, 5 \mathrm{mmol}, 1$ equiv) and diphenylphosphoryl azide $(1.65 \mathrm{~g}, 1.30 \mathrm{ml}, 6 \mathrm{mmol}, 1.2$ equiv) in toluene was added DBU (913 mg, $0.9 \mathrm{ml}, 6 \mathrm{mmol}, 1.2$ equiv) at $0{ }^{\circ} \mathrm{C}$ under an argon atmosphere. The reaction mixture was stirred for $12 \mathrm{~h}$ at room temperature. The mixture was washed with $\mathrm{H}_{2} \mathrm{O}, 1 \mathrm{M}$ aqueous $\mathrm{HCl}$, brine, dried over $\mathrm{Na}_{2} \mathrm{SO}_{4}$, filtered and concentrated under reduced pressure to afford a crude mixture as light yellow oil. The crude mixture was purified by flash column chromatography over silica gel ( $n$-hexane/EtOAc 95:5) afforded the product as colorless oil in $48 \%$ yield $(331 \mathrm{mg})$. The characterization data was consistent to that reported in the literature. ${ }^{41} \mathbf{H}$ NMR $\left(400 \mathrm{MHz}, C D C l_{3}\right) \delta 7.31(\mathrm{~d}, J=5.2 \mathrm{~Hz}, 1 \mathrm{H}), 7.10$ $6.96(\mathrm{~m}, 2 \mathrm{H}), 4.48(\mathrm{~s}, 2 \mathrm{H}) ;{ }^{13} \mathbf{C}$ NMR $\left(101 \mathrm{MHz}, C D C l_{3}\right) \delta 137.4,127.5,127.3,126.6$, 49.2 .

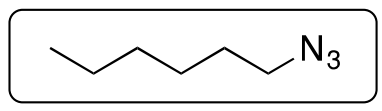

Hexyl azide (1r)

This procedure was adapted from the literature. ${ }^{5}$ To a solution of 1-bromohexane ( 825 $\mathrm{mg}, 5 \mathrm{mmol}, 1$ equiv) in DMF ( $2 \mathrm{~mL})$ was added $\mathrm{NaN}_{3}\left(488 \mathrm{mg}, 1.5\right.$ equiv) at $0{ }^{\circ} \mathrm{C}$. After stirring at room temperature for 6 hours, the mixture was diluted with $\mathrm{H}_{2} \mathrm{O}$ then extracted

\footnotetext{
${ }^{3}$ Rogers,S. A.; Melander, C. Angew. Chem. Int. Ed. 2008. 47. 5229-5231

${ }^{4}$ Pramanik, S.; Ghorai, P. Org. Lett. 2014, 16, 2104-2107.

${ }^{5}$ Ngai, M. H.; Yang, P.-Y.; Liu, K.; Shen, Y.; Wenk, M. R.; Yao, S. Q.; Lear, M. J. Chem. Comm. 2010, $46,8335-8337$.
} 
with a mixture of hexane/EtOAc (5:1). Combined organic layer was wash with $\mathrm{H}_{2} \mathrm{O}$, brine then dried over $\mathrm{Na}_{2} \mathrm{SO}_{4}$, filtered, concentrated under reduced pressure to give the product as colorless oil in $80 \%$ yield $(101.7 \mathrm{mg})$. The characterization data was consistent to that reported in the literature. ${ }^{1} \mathbf{H}$ NMR $\left(500 \mathrm{MHz}, C D C l_{3}\right): \delta 3.25(\mathrm{t}, J=7.0 \mathrm{~Hz}, 3 \mathrm{H})$, $1.66-1.54(\mathrm{~m}, 6 \mathrm{H}), 1.42-1.28(\mathrm{~m}, 2 \mathrm{H}), 0.90(\mathrm{t}, J=7.3,6.9 \mathrm{~Hz}, 2 \mathrm{H}) ;{ }^{13} \mathbf{C}$ NMR $(126$ $\left.\mathrm{MHz}, \mathrm{CDCl}_{3}\right): \delta 51.5,31.3,28.8,26.4,22.5,13.9$.

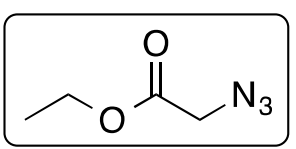

\section{Ethyl 2-azidoacetate (1s)}

According to general procedure $\mathrm{B}$, ethyl bromoacetate $(0.875 \mathrm{~g}, 552 \mathrm{uL}, 5 \mathrm{mmol}, 1$ equiv) was dissolved in DMSO (7.5 mL) and $\mathrm{NaN}_{3}$ (488 mg, $7.5 \mathrm{mmol}, 1.5$ equiv) was added slowly and reaction was stirred over night. Subsequent work up afforded the product as clear oil in $70 \%$ yield $(451 \mathrm{mg})$. The characterization data was consistent to that reported in the literature. ${ }^{6} \mathbf{H}$ NMR $\left(300 \mathrm{MHz}, C D C l_{3}\right): \delta 4.27(\mathrm{q}, J=7.2 \mathrm{~Hz}, 2 \mathrm{H})$, $3.86(\mathrm{~s}, 2 \mathrm{H}), 1.32(\mathrm{t}, J=7.2 \mathrm{~Hz}, 3 \mathrm{H}) ;{ }^{13} \mathbf{C}$ NMR $\left(126 \mathrm{MHz}, C D C l_{3}\right): \delta 168.3,61.9,50.4$, 14.1.<smiles>COc1cc(N)cc(OC)c1</smiles>

1-azido-3,5-dimethoxybenzene (1t)

In a round bottom flask 3,5-dimethoxyaniline (153 $\mathrm{mg}, 1 \mathrm{mmol}, 1$ equiv) was dissolved in $10 \%$ aqueous $\mathrm{HCl}(4 \mathrm{~mL})$. To the solution at $0^{\circ} \mathrm{C} \mathrm{NaNO}_{2}(69 \mathrm{mg}, 1 \mathrm{mmol}, 1$ equiv) was added dropwise, stirred for 10 minutes, and then $\mathrm{NaN}_{3}$ ( $85 \mathrm{mg}, 1.3 \mathrm{mmol}, 1.3$ equiv) was added in portions. The solution was allowed to warm to room temperature and stirred for 1 hour. Reaction was diluted with $\mathrm{H}_{2} \mathrm{O}$, extracted with $\mathrm{Et}_{2} \mathrm{O}$, washed with water, brine, and the combined organic layers were dried $\left(\mathrm{Na}_{2} \mathrm{SO}_{4}\right)$ and concentrated in vacuo to afford the product as a light yellow solid in $88 \%$ yield $(153 \mathrm{mg}) .{ }^{1} \mathbf{H}$ NMR $(400 \mathrm{MHz}$, $\left.C D l_{3}\right): \delta 6.25(\mathrm{t}, J=2.2 \mathrm{~Hz}, 1 \mathrm{H}), 6.19(\mathrm{~d}, J=2.1 \mathrm{~Hz}, 2 \mathrm{H}), 3.78(\mathrm{~s}, 6 \mathrm{H}) ;{ }^{13} \mathbf{C}$ NMR $(126$ $\left.\mathrm{MHz}, C D C l_{3}\right): \delta 161.8,142.1,97.6,97.4,55.6$; IR $\left(C D C l_{3}\right): 3003,2960,2939,2839$, 2108, 1593, 1475, 1444, 1427, 1253, 1205, 1155, 1066, $1049 \mathrm{~cm}^{-1}$; m.p.: $41-42^{\circ} \mathrm{C}$; HRMS (ESI): calc'd for $\mathrm{C}_{8} \mathrm{H}_{10} \mathrm{~N}_{3} \mathrm{O}_{3}(\mathrm{M}+\mathrm{H})^{+}$: 180.0773; Found: 180.0765 .

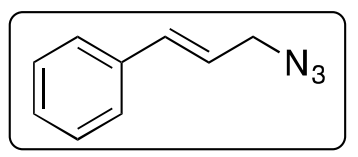

(E)-(3-Azidoprop-1-en-1-yl)benzene (1u)

According to a modified general procedure $\mathrm{B}$, cinnamyl bromide (788 $\mathrm{mg}, 4 \mathrm{mmol}, 1$ equiv) was dissolved in acetone $/ \mathrm{H}_{2} \mathrm{O}(15 \mathrm{~mL}, 4: 1)$ and $\mathrm{NaN}_{3}(520 \mathrm{mg}, 8 \mathrm{mmol}, 2$ equiv) was added slowly. After 1.5 hours and subsequent work up the reaction afforded the

\footnotetext{
${ }^{6}$ Gann, A. W.; Amoroso, J. W.; Einck, V. J.; Rice, W. P.; Chambers, J. J.; Schnarr, N. A. Org. Lett. 2014, $16,2003-2005$.
} 
product as brown oil in $90 \%$ yield $(570 \mathrm{mg})$. The characterization data was consistent to that reported in the literature. ${ }^{6}$

${ }^{1} \mathbf{H}$ NMR $\left(400 \mathrm{MHz}, C D C l_{3}\right): \delta 7.46-7.38(\mathrm{~m}, 2 \mathrm{H}), 7.37-7.31(\mathrm{~m}, 2 \mathrm{H}), 7.30-7.26(\mathrm{~m}$, $1 \mathrm{H}), 6.66(\mathrm{~d}, J=15.8 \mathrm{~Hz}, 1 \mathrm{H}), 6.25(\mathrm{dt}, J=15.7,6.6 \mathrm{~Hz}, 1 \mathrm{H}), 3.95(\mathrm{~d}, J=6.6 \mathrm{~Hz}, 2 \mathrm{H})$;

${ }^{13}$ C NMR (126 MHz, $\left.C D C l_{3}\right): \delta 136.0,134.5,128.7,128.2,126.6,122.4,53.0$.

\section{Synthesis of Iodoalkynes}

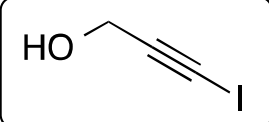

\section{3-Iodoprop-2-yn-1-ol (2a)}

According to general procedure A, propargyl alcohol (5.88 mL, $102 \mathrm{mmol}, 1$ equiv), $\mathrm{I}_{2}$ (28.5 g, $112 \mathrm{mmol}, 1.1$ equiv), and $\mathrm{KOH}$ (14.4 g, $256 \mathrm{mmol}, 2.5$ equiv) in $\mathrm{H}_{2} \mathrm{O}(20 \mathrm{~mL})$ were dissolved in $\mathrm{MeOH}(100 \mathrm{~mL})$ and stirred at room temperature for 12 hours. Subsequent workup and purification by flash column chromatography ( $n$-hexane:EtOAc 4:1) afforded the product as a light yellow solid in $65 \%$ yield $(12.1 \mathrm{~g})$. The characterization data was consistent to that reported in the literature. ${ }^{7}{ }^{1} \mathbf{H}$ NMR (400 MHz, $\left.C D C l_{3}\right): \delta 4.43(\mathrm{~d}, J=5.6 \mathrm{~Hz}, 2 \mathrm{H}) 1.63(\mathrm{t}, J=5.6 \mathrm{~Hz}, 1 \mathrm{H}) ;{ }^{13} \mathbf{C}$ NMR $(101 \mathrm{MHz}$, $\left.\mathrm{CDCl}_{3}\right): \delta 92.6,52.6,2.8$.

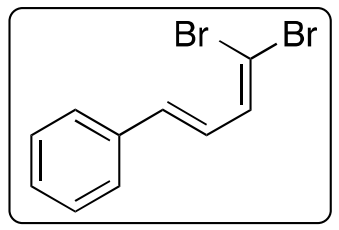

(E)-(4,4-dibromobuta-1,3-dien-1-yl)benzene (2j')

This procedure was adapted from the literature. ${ }^{8}$ To a solution of $\mathrm{PPh}_{3}(8.85 \mathrm{~g}, 33.8$ mmol, 4.5 equiv) in $\mathrm{CH}_{2} \mathrm{Cl}_{2}(30 \mathrm{~mL}), \mathrm{CBr}_{4}(6.32 \mathrm{~g}, 18.8 \mathrm{mmol}, 2.5$ equiv) was added slowly at $0^{\circ} \mathrm{C}$ under an argon atmosphere. After stirring for 30 minutes a solution of cinnamaldehyde $\left(1 \mathrm{~g}, 7.5 \mathrm{mmol}, 1\right.$ equiv) in $\mathrm{CH}_{2} \mathrm{Cl}_{2}(15 \mathrm{~mL})$ was added. The reaction was stirred for 1 hour at room temperature then filtered through celite, washed with aqueous $\mathrm{NaHCO}_{3}$, brine and concentrated in vacuo. Purification by flash column chromatography ( $n$-hexane:EtOAc 20:1) afforded the product as a white solid in $98 \%$ yield $(2.14 \mathrm{~g})$. The characterization data was consistent to that reported in the literature. ${ }^{9}$ ${ }^{1} \mathbf{H}$ NMR $\left(400 \mathrm{MHz}, C D C l_{3}\right): \delta 7.45(\mathrm{~d}, J=6.9 \mathrm{~Hz}, 2 \mathrm{H}), 7.32(\mathrm{~m}, 3 \mathrm{H}), 7.09(\mathrm{~d}, J=9.6$ $\mathrm{Hz}, 1 \mathrm{H}), 6.75(\mathrm{~m}, 2 \mathrm{H}) ;{ }^{13} \mathbf{C}$ NMR $\left(101 \mathrm{MHz}, C D C l_{3}\right): \delta 137.1,136.3,135.7,128.8$, $128.6,126.9,125.3,91.3$.

\footnotetext{
7 Jahnke, E.; Weiss, J.; Neuhaus, S.; Hoheisel, T. N.; Frauenrath, H. Chem. Eur. J. 2009, 15, 388-404.

${ }^{8}$ Rouden, J.; Seitz, T.; Lemoucheux, L.; Lasne, M.-C. J. Org. Chem. 2004, 69, 3787-3793.

${ }^{9}$ Chen, X. Y.; Wang, L.; Frings, M.; Bolm, C. Org. Lett. 2014, 16, 3796-3799.
} 


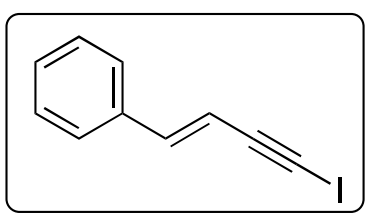

1-iodo-2-styrylacetylene $(2 \mathrm{j})$

Under an argon atmosphere $n$-BuLi $(7.52 \mathrm{~mL}, 13.8 \mathrm{mmol} .2$ equiv) was added dropwise at $-78^{\circ} \mathrm{C}$ to a solution of $(E)$-(4,4-dibromobuta-1,3-dien-1-yl)benzene (2.0 g, $6.9 \mathrm{mmol}, 1$ equiv) in THF (23 mL). After stirring for 75 minutes the reaction was warmed to $0^{\circ} \mathrm{C}$ and a solution of $\mathrm{I}_{2}(1.93 \mathrm{~g}, 7.6 \mathrm{mmol}, 1.1$ equiv) in THF $(12 \mathrm{~mL})$ was added slowly. The reaction mixture was allowed to warm to r.t. After stirring for 16 hours the reaction was quenched with aqueous $\mathrm{Na}_{2} \mathrm{~S}_{2} \mathrm{O}_{3}$ and extracted with $\mathrm{Et}_{2} \mathrm{O}$. The combined organic layers were washed with water, dried $\left(\mathrm{MgSO}_{4}\right)$ and concentrated to afford the product as a light yellow solid in $40 \%$ yield $(700 \mathrm{mg})$. The characterization data was consistent to that reported in the literature. ${ }^{10} \mathbf{H}$ NMR $\left(400 \mathrm{MHz}, C D C l_{3}\right): \delta 7.48-7.28(\mathrm{~m}, 5 \mathrm{H}), 6.99(\mathrm{~d}, J$ $=16.3 \mathrm{~Hz}, 1 \mathrm{H}), 6.27(\mathrm{~d}, J=16.3 \mathrm{~Hz}, 1 \mathrm{H}) .{ }^{13} \mathbf{C ~ N M R}\left(101 \mathrm{MHz}, C D C l_{3}\right): \delta 143.6,135.6$, $129.0,128.7,126.4,108.2,93.4,7.9$.<smiles></smiles>

\section{Naphthalen-2-yl 3-iodopropiolate (2k)}

According to general procedure $\mathrm{A}$, naphthalene-2-yl propiolate $(0.20 \mathrm{~g}, 1 \mathrm{mmol}, 1.0$ equiv), $\mathrm{I}_{2}\left(0.28 \mathrm{~g}, 1.1 \mathrm{mmol}, 1.1\right.$ equiv), and $\mathrm{KOH}\left(0.14 \mathrm{~g}, 2.5 \mathrm{mmol}, 2.5\right.$ equiv) in $\mathrm{H}_{2} \mathrm{O}$ $(0.5 \mathrm{~mL})$ were dissolved in $\mathrm{MeOH}(2 \mathrm{~mL})$ and stirred at room temperature for 12 hours. Subsequent workup and purification by flash column chromatography ( $n$-hexane: $\mathrm{Et}_{2} \mathrm{O}$ 9:1) afforded the product as an off white solid in $89 \%$ yield $(0.29 \mathrm{~g})$. ${ }^{1} \mathbf{H}$ NMR (500 $\left.\mathrm{MHz}, C D C l_{3}\right): \delta 7.92-7.79(\mathrm{~m}, 3 \mathrm{H}), 7.62(\mathrm{dd}, J=2.3 \mathrm{~Hz}, 0.6 \mathrm{~Hz}, 1 \mathrm{H}), 7.54-7.45(\mathrm{~m}$, $2 \mathrm{H}), 7.26(\mathrm{dd}, J=8.9,2.4 \mathrm{~Hz}, 1 \mathrm{H}) ;{ }^{13} \mathbf{C}$ NMR $\left(126 \mathrm{MHz}, C D C l_{3}\right): \delta 150.6,147.5,133.6$, $131.7,129.7,127.8,127.8,126.8,126.1,120.4,118.5,86.6,16.7$; IR $\left(C D C l_{3}\right): 2174$, 1705, 1701, 1689, 1244, 1211, 1152, 893, 856, 735, $432 \mathrm{~cm}^{-1}$; m.p.: 117-118 ${ }^{\circ}$; HRMS (ESI): calc'd for $\mathrm{C}_{13} \mathrm{H}_{11} \mathrm{INO}_{2}(\mathrm{M}+\mathrm{H})^{+}:$339.9491; Found: 339.9835 .

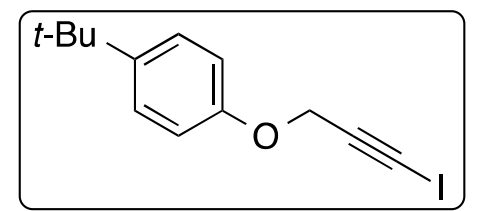

1-(tert-butyl)-4-((3-iodoprop-2-yn-1-yl)oxy)benzene (21)

This procedure was adapted from the literature. ${ }^{11}$ In a round bottom flask containing a stir bar 1-(tert-butyl)-4-(prop-2-yn-1-yloxy)benzene (1.05 g, $5.6 \mathrm{mmol}, 1$ equiv) was dissolved in dry THF $(56 \mathrm{~mL})$ in the presence of $4 \AA \mathrm{MS}$. To the stirring reaction mixture was added $\mathrm{CuI}$ (57 mg, $5 \mathrm{~mol} \%$ ) and $N$-iodomorpholine hydriodide (3.62 g, $10.6 \mathrm{mmol}, 2$

${ }^{10}$ Pelletier, G.; Lie, S.; Mousseau, J. J.; Charette, A. B. Org. Lett. 2012, 14, 5464-5467.

${ }^{11}$ Hein, J. E.; Tripp, J. C.; Krasnova, L. B.; Sharpless, K. B.; Fokin, V. V. Angew. Chem. Int. Ed. 2009, 48, 8018-8021. 
equiv). After stirring in dark at room temperature for 12 hours the reaction mixture was quenched with saturated aqueous $\mathrm{NH}_{4} \mathrm{Cl}$ and extracted with $\mathrm{Et}_{2} \mathrm{O}$. The combined organic layers were washed with saturated aqueous $\mathrm{Na}_{2} \mathrm{~S}_{2} \mathrm{O}_{3}$ and brine, dried over $\mathrm{Na}_{2} \mathrm{SO}_{4}$, filtered, and concentrated in vacuo to afford the product as an amber oil in $99 \%$ yield $(1.74 \mathrm{~g})$. The characterization data was consistent to that reported in the literature. ${ }^{12}{ }^{1} \mathbf{H}$ NMR (500 MHz, $C D C l 3$ ): $\delta 7.33$ (dd, $J=9.0 \mathrm{~Hz}, 2 \mathrm{H}), 6.91(\mathrm{dd}, J=9.0 \mathrm{~Hz}, 2 \mathrm{H}), 4.81$ (s, 2H), $1.32(\mathrm{~s}, 9 \mathrm{H}) ;{ }^{13} \mathbf{C}$ NMR $\left(126 \mathrm{MHz}, \mathrm{CDCl}_{3}\right): 155.4,144.3,126.3,114.3,89.4,57.4$, $34.1,31.5,4.8$.

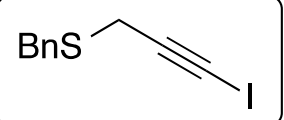

benzyl(3-iodoprop-2-yn-1-yl)sulfane (2m)

This procedure was adapted from the literature. ${ }^{11}$ To a solution of benzyl(prop-2yn-1yl)sulfone (405 mg, $2.5 \mathrm{mmol}, 1$ equiv) in THF $(6 \mathrm{~mL})$ was added $N$-iodomorpholine

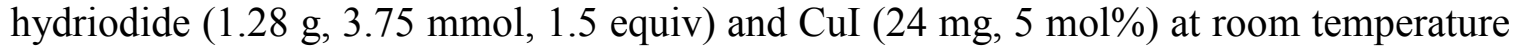
under an argon atmosphere. After stirring at room temperature for 24 hours the mixture was diluted with $\mathrm{H}_{2} \mathrm{O}$ and extracted with EtOAc. Combined organic layers were washed with aqueous $\mathrm{Na}_{2} \mathrm{~S}_{2} \mathrm{O}_{3}$ solution, brine, and then died over $\mathrm{Na}_{2} \mathrm{SO}_{4}$, filtered, concentrated under reduced pressure to afford a crude mixture of the product. The crude mixture was purified by column chromatography over silica gel with $n$-hexane $(100 \%)$ afforded the product as a yellow solid in $87 \%$ yield $(624 \mathrm{mg}) .{ }^{1} \mathbf{H}$ NMR $\left(500 \mathrm{MHz}, C D C l_{3}\right): \delta 7.36-$ $7.22(\mathrm{~m}, 5 \mathrm{H}), 3.85(\mathrm{~s}, 2 \mathrm{H}), 3.23(\mathrm{~s}, 2 \mathrm{H}) ;{ }^{13} \mathbf{C}$ NMR $\left(126 \mathrm{MHz}, C D C l_{3}\right): \delta 137.5,129.1$, 128.7, 127.4, 89.9, 35.7, 20.7, -1.6; IR: 3300, 3064, 2944, 2911, 2854, 1964, 1601, 1493, 1452, 1438, 1403, 1244, 1229, 1197, 1172, 1150, 1069, 1019, 923, $898 \mathrm{~cm}^{-1}$; m.p.: 43-45 ${ }^{\circ} \mathrm{C}$; HRMS (ESI): calc'd for $\mathrm{C}_{10} \mathrm{H}_{10} \mathrm{IS}(\mathrm{M}+\mathrm{H})^{+}: 288.9548$; Found: 288.9552.

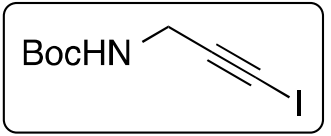

\section{tert-butyl (3-iodoprop-2-yn-1-yl)carbamate (2n)}

According to general procedure A, $N$-Boc propargyl amine (2.32 g, $15 \mathrm{mmol}, 1.0$ equiv), $\mathrm{I}_{2}$ (4.2 g, $16.5 \mathrm{mmol}, 1.1$ equiv), and $\mathrm{KOH}\left(2.13,37.5 \mathrm{mmol}, 2.5\right.$ equiv) in $\mathrm{H}_{2} \mathrm{O}$ (4.5 mL) were dissolved in $\mathrm{MeOH}(21 \mathrm{~mL})$ and stirred at room temperature for 24 hours. Subsequent work up and purification by flash column chromatography produced the product as an off white solid in $84 \%$ yield $(3.55 \mathrm{~g}) .{ }^{1} \mathbf{H}$ NMR $\left(500 \mathrm{MHz}, C D C l_{3}\right): \delta 4.70$ $(\mathrm{s}, 1 \mathrm{H}), 4.07(\mathrm{~d}, J=3.6 \mathrm{~Hz}, 2 \mathrm{H}), 1.45(\mathrm{~s}, 9 \mathrm{H}) ;{ }^{13} \mathbf{C}$ NMR $\left(126 \mathrm{MHz}, C D C l_{3}\right): \delta 155.3$, 90.5, 80.3, 32.4, 28.5, 28.4; IR $\left(\mathrm{NaCl}, C D C l_{3}\right)$ : 3337, 2978, 2932, 1699, 1524, 1393, 1279, 1252, 1165, 1049, 914, $858 \mathrm{~cm}^{-1}$; m.p.: 124-125 ${ }^{\circ} \mathrm{C}$; HRMS (TOF, ESI+): calc'd for $\mathrm{C}_{8} \mathrm{H}_{12} \mathrm{INNaO}_{2}(\mathrm{M}+\mathrm{Na})^{+}: 303.9805$; Found: 303.9810 .

\footnotetext{
${ }^{12}$ Schulman, J. M.; Friedman, A. A.; Panteleev, J.; Lautens, M. Chem. Commun. 2012, 48, 55-57.
} 


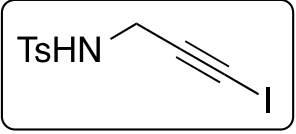

N-(3-iodoprop-2-yn-1-yl)-4-methylbenzenesulfonamide (2p)

According to general procedure A, $N$-tosyl propargyl amine (2.09 g, $10 \mathrm{mmol}, 1.0$ equiv), $\mathrm{I}_{2}$ (2.81 g, $11 \mathrm{mmol}, 1.1$ equiv), and $\mathrm{KOH}$ (1.42 g, $25 \mathrm{mmol}, 2.5$ equiv) in $\mathrm{H}_{2} \mathrm{O}(3 \mathrm{~mL})$ were dissolved in $\mathrm{MeOH}(14 \mathrm{~mL})$ and stirred at room temperature for 24 hours. Subsequent workup and purification by flash column chromatography afforded the product as an off white solid in $89 \%$ yield $(2.98 \mathrm{~g})$. The characterization data was consistent to that reported in the literature. ${ }^{12}$

${ }^{1} \mathbf{H}$ NMR (400 MHz, $\left.C D C l_{3}\right): \delta 7.72(\mathrm{~d}, J=8.2 \mathrm{~Hz}, 2 \mathrm{H}), 7.34(\mathrm{~d}, J=8.1 \mathrm{~Hz}, 2 \mathrm{H}), 3.99$ $(\mathrm{d}, J=6.5 \mathrm{~Hz}, 2 \mathrm{H}), 2.45(\mathrm{~s}, 3 \mathrm{H}) ;{ }^{13} \mathrm{C}$ NMR $\left(101 \mathrm{MHz}, C D C l_{3}\right): \delta 142.3,135.0,128.2$, $128.1,126.0,86.4,33.0,20.1$

\section{Synthesis of Terminal alkyne}

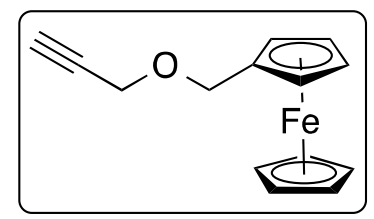

propargyloxymethylferrocene $(3 \mathrm{~g})$

This product was synthesized according to literature procedures. ${ }^{13}$ To a suspension of sodium hydride (60\% dispersion in mineral oil, $80 \mathrm{mg}, 2 \mathrm{mmol}, 2$ equiv.) in DMF/toluene $(1: 1,10 \mathrm{~mL})$ was added ferrocenylmethyl alcohol (216 $\mathrm{mg}, 1 \mathrm{mmol}, 1$ equiv) and propargyl bromide ( $80 \%$ wt. in toluene, $150 \mathrm{uL}, 1.4$ equiv) at $0{ }^{\circ} \mathrm{C}$ under an argon atmosphere. After stirring 3 hours at room temperature, the reaction was quenched with $\mathrm{H}_{2} \mathrm{O}$ and extracted with $\mathrm{Et}_{2} \mathrm{O}$. Combined organic layer were washed with brine, then dried over $\mathrm{MgSO}_{4}$, filtered, concentrated under reduced pressure. Purification by flash column chromatography by flash column chromatography with $n$-hexane/EtOAc $(9: 1)$ afforded the title compound in $98 \%$ yield $(249 \mathrm{mg})$. The characterization data was consistent with that reported in the literature. ${ }^{1} \mathbf{H}$ NMR (400 MHz, CDCl3): $\delta 4.39$ (s, $2 \mathrm{H}), 4.25(\mathrm{t}, J=1.9 \mathrm{~Hz}, 2 \mathrm{H}), 4.16(\mathrm{t}, J=1.9 \mathrm{~Hz}, 2 \mathrm{H}), 4.15(\mathrm{~s}, 5 \mathrm{H}), 4.12(\mathrm{~d}, \mathrm{~J}=2.4 \mathrm{~Hz}$, 2H), $2.45-2.43(\mathrm{~m}, 1 \mathrm{H}) ;{ }^{13} \mathbf{C}$ NMR $\left(101 \mathrm{MHz}, C D C l_{3}\right): \delta 82.5,80.1,74.5,69.7,68.8$, $68.6,67.8,56.6$.

\section{(MC) $)^{2}$ R Products}

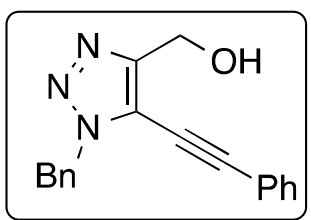

(1-benzyl-5-(phenylethynyl)-1H-1,2,3-triazol-4-yl)methanol (9a)

${ }^{13}$ Rajakumar, P.; Kannan, A.; Anandhan, R. New J. Chem. 2014, 38, 1594-1600 
According to general procedure $\mathrm{C}$ iodoalkyne $\mathbf{2 a}$ (36.4 $\mathrm{mg}, 0.20 \mathrm{mmol}, 1$ equiv), benzyl azide (27.4 mg, $0.22 \mathrm{mmol}, 1.1$ equiv), CuI (1.9 mg, $5 \mathrm{~mol} \%$ ), TBTA (5.3 mg, $5 \mathrm{~mol}$ $\%$ ), XPhos-Pd-G3 (1.7 mg, $1 \mathrm{~mol} \mathrm{\% ),} \mathrm{KOAc} \mathrm{(59} \mathrm{mg,} 0.6 \mathrm{mmol}, 3$ equiv) and phenylacetylene $(33 \mu \mathrm{L}, 0.30 \mathrm{mmol}, 1.5$ equiv) were dissolved in dry THF under argon. After stirring at room temperature for 4 hours the reaction was stirred at $85^{\circ} \mathrm{C}$ for 16 hours. The product was isolated through column chromatography ( $n$-hexane/EtOAc $=$ $6: 4)$ as a yellow solid in $73 \%$ yield $(42.1 \mathrm{mg}) .{ }^{1} \mathbf{H}$ NMR $\left(500 \mathrm{MHz}, C D C l_{3}\right): \delta 7.47(\mathrm{~m}$, 2H), $7.38(\mathrm{~m}, 8 \mathrm{H}), 5.61(\mathrm{~s}, 2 \mathrm{H}), 4.84(\mathrm{~s}, 2 \mathrm{H}), 2.64(\mathrm{~s}, 1 \mathrm{H},-\mathrm{OH}) ;{ }^{13} \mathbf{C}$ NMR $(126 \mathrm{MHz}$, $\left.\mathrm{CDCl}_{3}\right): \delta 149.3,134.5,131.6,129.7,128.9,128.6,128.6,128.2,121.1,119.4,102.0$, 73.3, 55.9, 53.1. IR (NaCl, $\left.C D C C_{3}\right): 3335,1683,1653,1456,1209,1120,1029,756,691$, 432, 407; m.p.: $119-120^{\circ} \mathrm{C}$; HRMS (ESI): calc'd for $\mathrm{C}_{18} \mathrm{H}_{16} \mathrm{~N}_{3} \mathrm{O}(\mathrm{M}+\mathrm{H})^{+}: 290.1293$; Found: 290.1284.

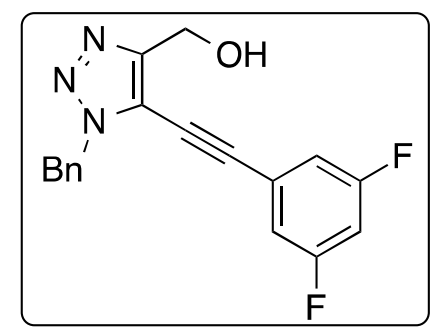

(1-benzyl-5-((3,5-difluorophenyl)ethynyl)-1H-1,2,3-triazol-4-yl)methanol (9b)

According to general procedure $C$ iodoalkyne $2 \mathbf{a}(45.5 \mathrm{mg}, 0.25 \mathrm{mmol}, 1$ equiv), benzyl azide (36.6 mg, $0.28 \mathrm{mmol}, 1.1$ equiv), CuI (2.3 mg, $5 \mathrm{~mol} \%$ ), TBTA (6.6 mg, $5 \mathrm{~mol}$ $\%$ ), XPhos-Pd-G3 (2.1 mg, $1 \mathrm{~mol} \%$ ), KOAc (74 mg, $0.75 \mathrm{mmol}, 3$ equiv) and 1-ethynyl3,5-difluorobenzene ( $45 \mu \mathrm{L}, 0.38 \mathrm{mmol}, 1.5$ equiv) were dissolved in dry THF under argon. After stirring at room temperature for 3 hours the reaction was stirred at $85^{\circ} \mathrm{C}$ for 24 hours. The product was isolated through column chromatography ( $n$-hexane/EtOAc $=$ $6: 4)$ as a light brown solid in $53 \%$ yield $(44.3 \mathrm{mg}) .{ }^{1} \mathbf{H}$ NMR $\left(500 \mathrm{MHz}, C D C l_{3}\right): \delta 7.41-$ $7.31(\mathrm{~m}, 5 \mathrm{H}), 6.99-6.93(\mathrm{~m}, 2 \mathrm{H}), 6.89(\mathrm{tt}, J=8.8,2.3 \mathrm{~Hz}, 1 \mathrm{H}), 5.62(\mathrm{~s}, 2 \mathrm{H}), 4.83(\mathrm{~d}, J=$ $5.6 \mathrm{~Hz}, 2 \mathrm{H}) ;{ }^{13} \mathbf{C}$ NMR $\left(126 \mathrm{MHz}, C D C l_{3}\right) \delta 163.7(\mathrm{~d}, J=13.2 \mathrm{~Hz}), 161.7(\mathrm{~d}, J=13.2$ $\mathrm{Hz}), 150.0,134.2,129.0,128.7,128.0,123.7$ (t, $J=11.7 \mathrm{~Hz}), 114.7$ (d, $J=6.9 \mathrm{~Hz})$, $114.5(\mathrm{~d}, J=6.7 \mathrm{~Hz}), 105.9(\mathrm{t}, J=25.3 \mathrm{~Hz}), 99.4(\mathrm{t}, J=4.0 \mathrm{~Hz}), 75.1,55.9,53.3 ;{ }^{19} \mathbf{F}$ NMR $\left(470 \mathrm{MHz}, C D C l_{3}\right):-108.36(\mathrm{t}, J=7.4 \mathrm{~Hz})$; IR (NaCl, $\left.C D C l_{3}\right): 3420,1653,1616$, 1589, 1429, 1269, 1219, 1125, 1030, 959, $772 \mathrm{~cm}^{-1}$; m.p.: 135-136 ${ }^{\circ} \mathrm{C}$; HRMS (ESI): calc'd for $\mathrm{C}_{18} \mathrm{H}_{14} \mathrm{~F}_{2} \mathrm{~N}_{3} \mathrm{O}(\mathrm{M}+\mathrm{H})^{+}: 326.1118$; Found: 326.1111 .

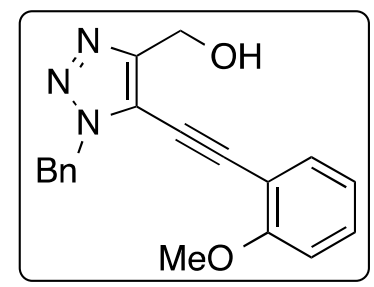

(1-benzyl-5-((2-methoxyphenyl)ethynyl)-1H-1,2,3-triazol-4-yl)methanol (9c)

According to general procedure $C$ iodoalkyne $2 \mathrm{a}(45.5 \mathrm{mg}, 0.25 \mathrm{mmol}, 1$ equiv), benzyl azide (36.6 mg, $0.28 \mathrm{mmol}, 1.1$ equiv), CuI (2.3 mg, $5 \mathrm{~mol} \%$ ), TBTA (6.6 mg, $5 \mathrm{~mol}$ $\%$ ), XPhos-Pd-G3 (2.1 mg, $1 \mathrm{~mol} \%)$, KOAc (74 mg, $0.75 \mathrm{mmol}, 3$ equiv) and 2ethynylanisole ( $48 \mu \mathrm{L}, 0.38 \mathrm{mmol}, 1.5$ equiv) were dissolved in dry THF under argon. 
After stirring at room temperature for 2 hours the reaction was stirred at $85^{\circ} \mathrm{C}$ for 24 hours. The product was isolated through column chromatography $(n$-hexane/EtOAc $=$ 6:4) as a dark brown solid in $71 \%$ yield $(56.5 \mathrm{mg}) .{ }^{1} \mathbf{H}$ NMR (500 MHz, $\left.C D C l_{3}\right): \delta 7.45$ $(\mathrm{m}, 2 \mathrm{H}), 7.42(\mathrm{dd}, J=7.8,1.5 \mathrm{~Hz}, 1 \mathrm{H}), 7.39$ (ddd, $J=8.4,7.5,1.7 \mathrm{~Hz}, 1 \mathrm{H}), 7.33(\mathrm{~m}, 3 \mathrm{H})$, $6.97(\mathrm{td}, J=7.5,1.0 \mathrm{~Hz}, 1 \mathrm{H}), 6.93(\mathrm{~d}, J=8.4 \mathrm{~Hz}, 1 \mathrm{H}), 5.61(\mathrm{~s}, 2 \mathrm{H}), 4.85(\mathrm{~d}, J=5.7 \mathrm{~Hz}$, $2 \mathrm{H}), 3.89(\mathrm{~s}, 3 \mathrm{H}) ;{ }^{13} \mathbf{C}$ NMR $\left(126 \mathrm{MHz}, \mathrm{CDCl}_{3}\right): \delta 160.3,149.2,134.7,133.0,131.2$, $128.8,128.5,128.4,120.6,119.7,110.7,110.5,99.0,77.4,56.2,55.8,52.8 ;$ IR $(\mathrm{NaCl}$, $\mathrm{CDCl}_{3}$ ): 3347, 2938, 2226, 1691, 1435, 1279, 1250, 1108, 1020, 756, $702 \mathrm{~cm}^{-1}$; m.p.: $119-120^{\circ} \mathrm{C}$; HRMS (ESI): calc'd for $\mathrm{C}_{19} \mathrm{H}_{18} \mathrm{~N}_{3} \mathrm{O}_{2}(\mathrm{M}+\mathrm{H})^{+}: 320.1399$; Found: 320.1401 .

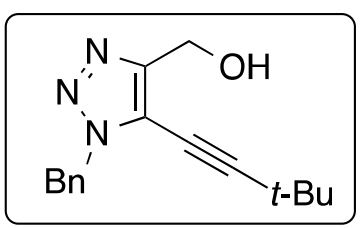

(1-benzyl-5-(3,3-dimethylbut-1-yn-1-yl)-1H-1,2,3-triazol-4-yl)methanol (9d)

According to general procedure $C$ iodoalkyne $2 \mathrm{a}(45.5 \mathrm{mg}, 0.25 \mathrm{mmol}, 1$ equiv), benzyl azide (36.6 mg, $0.28 \mathrm{mmol}, 1.1$ equiv), CuI (2.3 mg, $5 \mathrm{~mol} \%$ ), TBTA (6.6 mg, $5 \mathrm{~mol}$ $\%$ ), XPhos-Pd-G3 (2.1 mg, $1 \mathrm{~mol} \%$ ), KOAc (74 mg, $0.75 \mathrm{mmol}, 3$ equiv) and 3,3dimethylbut-1-yne ( $92 \mu \mathrm{L}, 0.75 \mathrm{mmol}, 3$ equiv) were dissolved in dry THF under argon. After stirring at room temperature for 2 hours the reaction was stirred at $85^{\circ} \mathrm{C}$ for 24 hours. The product was isolated through column chromatography $(n$-hexane/EtOAc $=$ $8: 2)$ as an off white solid in $80 \%$ yield $(54.0 \mathrm{mg}) .{ }^{1} \mathbf{H}$ NMR $\left(500 \mathrm{MHz}, C D C l_{3}\right): \delta 7.30$ $(\mathrm{m}, 5 \mathrm{H}), 5.48(\mathrm{~s}, 2 \mathrm{H}), 4.72(\mathrm{~d}, J=5.3 \mathrm{~Hz}, 2 \mathrm{H}), 3.51(\mathrm{~s}, 1 \mathrm{H},-\mathrm{OH}), 1.27(\mathrm{~s}, 9 \mathrm{H}) ;{ }^{13} \mathbf{C}$ NMR (126 MHz, $\left.C D C l_{3}\right): \delta 134.6,128.7,128.4,128.1,111.9,64.0,55.4,52.7,30.4,28.4$; IR $\left(\mathrm{NaCl}, \mathrm{CDCl}_{3}\right): 3347,3264,2970,1456,1364,1300,1219,1030,912,772,702 \mathrm{~cm}^{-1}$; m.p.: 106-108 ${ }^{\circ} \mathrm{C}$; HRMS (ESI): calc'd for $\mathrm{C}_{16} \mathrm{H}_{20} \mathrm{~N}_{3} \mathrm{O}(\mathrm{M}+\mathrm{H})^{+}$: 270.1528; Found: 270.1606 .

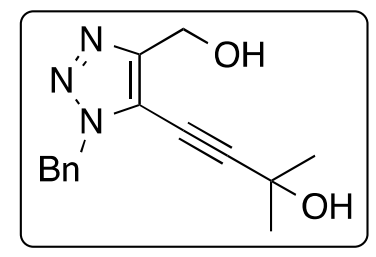

4-(1-benzyl-4-(hydroxymethyl)-1H-1,2,3-triazol-5-yl)-2-methylbut-3-yn-2-ol (9e)

According to general procedure C iodoalkyne 2a $(45.5 \mathrm{mg}, 0.25 \mathrm{mmol}, 1$ equiv), benzyl azide (36.6 mg, $0.28 \mathrm{mmol}, 1.1$ equiv), CuI (2.3 mg, $5 \mathrm{~mol} \%$ ), TBTA (6.6 mg, $5 \mathrm{~mol}$ $\%$ ), XPhos-Pd-G3 (2.1 mg, $1 \mathrm{~mol} \%$ ), KOAc (74.0 mg, $1.5 \mathrm{mmol}, 3$ equiv) and 2-methyl3-butyn-2-ol (73 $\mu \mathrm{L}, 0.75 \mathrm{mmol}, 1.5$ equiv) were dissolved in dry THF under argon. After stirring at room temperature for 3 hours the reaction was stirred at $85^{\circ} \mathrm{C}$ for 24 hours. The product was isolated through column chromatography $(n$-hexane/EtOAc $=$ 9:1) as a light brown solid in $80 \%$ yield $(54.4 \mathrm{mg}) .{ }^{1} \mathbf{H}$ NMR (500 MHz, $\left.C D C l_{3}\right): \delta 7.29$ (m, 5H), $5.48(\mathrm{~s}, 2 \mathrm{H}), 4.68(\mathrm{~d}, J=1.6 \mathrm{~Hz}, 2 \mathrm{H}), 3.95(\mathrm{~s}, 1 \mathrm{H},-\mathrm{OH}), 3.86(\mathrm{~s}, 1 \mathrm{H},-\mathrm{OH}), 1.54$ (s, 6H); ${ }^{13}$ C NMR (126 MHz, $C D C l$ ): $\delta$ 149.4, 134.3, 129.1, 128.8, 128.5, 128.1, 119.5, 107.6, 66.6, 65.3, 55.5, 53.0, 30.8; IR ( NaCl, $\left.C D C l_{3}\right): 3347,2982,2933,1667,1456$, 1364, 1292, 1169, 1024, 966, 735, $409 \mathrm{~cm}^{-1}$; m.p.: $74-75^{\circ} \mathrm{C}$; HRMS (ESI): calc'd for $\mathrm{C}_{15} \mathrm{H}_{18} \mathrm{~N}_{3} \mathrm{O}_{2}(\mathrm{M}+\mathrm{H})^{+}: 272.1321$; Found: 272.1399 . 


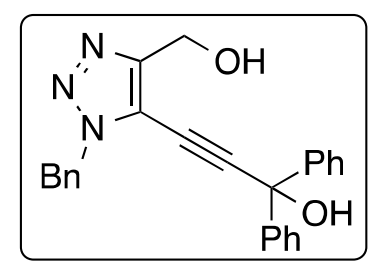

3-(1-benzyl-4-(hydroxymethyl)-1H-1,2,3-triazol-5-yl)-1,1-diphenylprop-2-yn-1-ol (9f) According to general procedure $C$ iodoalkyne $2 \mathrm{a}(45.5 \mathrm{mg}, 0.25 \mathrm{mmol}, 1$ equiv), benzyl azide (36.6 mg, $0.28 \mathrm{mmol}, 1.1$ equiv), CuI (2.3 mg, $5 \mathrm{~mol} \%$ ), TBTA (6.6 mg, $5 \mathrm{~mol}$ $\%$ ), XPhos-Pd-G3 (2.1 mg, $1 \mathrm{~mol} \%$ ), KOAc (74 mg, $0.75 \mathrm{mmol}, 3$ equiv) and 1,1diphenylprop-2-yn-1-ol ( $78 \mathrm{mg}, 0.38 \mathrm{mmol}, 1.5$ equiv) were dissolved in dry THF under argon. After stirring at room temperature for 2 hours the reaction was stirred at $85^{\circ} \mathrm{C}$ for 21 hours. The product was isolated through column chromatography ( $n$-hexane/EtOAc $=$ $6: 4)$ as an off white solid in $76 \%$ yield $(75.7 \mathrm{mg}) .{ }^{1} \mathbf{H}$ NMR $\left(500 \mathrm{MHz}, C D C l_{3}\right): \delta 7.52-$ $7.43(\mathrm{~m}, 4 \mathrm{H}), 7.31-7.26(\mathrm{~m}, 6 \mathrm{H}), 7.26-7.17(\mathrm{~m}, 3 \mathrm{H}), 7.10-7.04(\mathrm{~m}, 2 \mathrm{H}), 5.39(\mathrm{~s}, 2 \mathrm{H})$, $4.60(\mathrm{~s}, 2 \mathrm{H}) ;{ }^{13} \mathrm{C}$ NMR $\left(126 \mathrm{MHz}, C D C l_{3}\right): \delta 144.0,134.2,128.8,128.5,128.4,128.0$, 128.0, 126.1, 105.5, 74.7, 71.3, 55.5, 53.0; IR (NaCl, $\left.C D C l_{3}\right): 3360,2361,2340,1734$, 1684, 1456, 1219, 1030, 772, 733, $698 \mathrm{~cm}^{-1}$; m.p.: 98-99 ${ }^{\circ} \mathrm{C}$; HRMS (ESI): calc'd for $\mathrm{C}_{25} \mathrm{H}_{22} \mathrm{~N}_{3} \mathrm{O}_{2}(\mathrm{M}+\mathrm{H})^{+}:$396.1634; Found: 396.1712 .

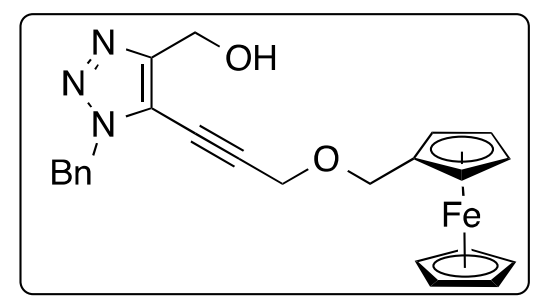

(1-benzyl-5-(3-(ferrocenyloxy)prop-1-yn-1-yl)-1H-1,2,3-triazol-4-yl)methanol (9g)

According to general procedure $C$ iodoalkyne $2 \mathbf{a}(45.5 \mathrm{mg}, 0.25 \mathrm{mmol}, 1$ equiv), benzyl azide (36.6 mg, $0.28 \mathrm{mmol}, 1.1$ equiv), CuI (2.3 mg, $5 \mathrm{~mol} \%$ ), TBTA (6.6 mg, $5 \mathrm{~mol}$ \%), XPhos-Pd-G3 (2.1 mg, $1 \mathrm{~mol} \%)$, KOAc (74 mg, $0.75 \mathrm{mmol}, 3$ equiv) and terminal alkyne $3 \mathrm{~g}$ (95 mg, $0.38 \mathrm{mmol}, 1.5$ equiv) were dissolved in dry THF under argon. After stirring at room temperature for 4 hours the reaction was stirred at $85^{\circ} \mathrm{C}$ for 24 hours. The product was isolated through column chromatography ( $n$-hexane/EtOAc $=1: 1)$ as a orange solid in $52 \%$ yield $(56.8 \mathrm{mg}){ }^{1} \mathbf{H}$ NMR $\left(400 \mathrm{MHz}, C D C l_{3}\right): \delta 7.40-7.27(\mathrm{~m}, 5 \mathrm{H})$, $5.55(\mathrm{~s}, 2 \mathrm{H}), 4.79(\mathrm{~d}, J=6.4 \mathrm{~Hz}, 2 \mathrm{H}), 4.37(\mathrm{~s}, 2 \mathrm{H}), 4.35(\mathrm{~s}, 2 \mathrm{H}), 4.22(\mathrm{t}, J=1.9 \mathrm{~Hz}, 2 \mathrm{H})$, $4.17(\mathrm{t}, J=1.8 \mathrm{~Hz}, 2 \mathrm{H}), 4.13(\mathrm{~s}, 5 \mathrm{H}) ;{ }^{13} \mathbf{C} \mathbf{N M R}\left(101 \mathrm{MHz}, C D C l_{3}\right): \delta 149.9,134.5$, 129.0, 128.7, 128.1, 119.1, 99.3, 70.7, 69.7, 68.9, 68.7, 68.3, 57.0, 55.8, 53.0; IR (ATR): 3237, 3103, 2923, 2869, 2848, 1456, 1371, 1349, 1332, 1295, 1239, 1213, 1104, 1063, 1037, 100, 965, 906, 837, $811 \mathrm{~cm}^{-1}$; m.p.: 108-109 ${ }^{\circ} \mathrm{C}$; HRMS (ESI): calc'd for $\mathrm{C}_{24} \mathrm{H}_{24} \mathrm{FeN}_{3} \mathrm{O}_{2}(\mathrm{M}+\mathrm{H})^{+}: 442.1218$; Found: 442.1213.

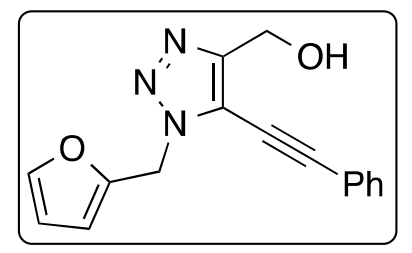




\section{(1-(furan-2-ylmethyl)-5-(phenylethynyl)-1H-1,2,3-triazol-4-yl)methanol (9h)}

According to general procedure $\mathrm{C}$ iodoalkyne $\mathbf{2 a}(45.5 \mathrm{mg}, 0.25 \mathrm{mmol}, 1$ equiv), azide $\mathbf{1 h}$ (33.9 mg, $0.28 \mathrm{mmol}, 1.1$ equiv), CuI (2.3 mg, $5 \mathrm{~mol} \%$ ), TBTA (6.6 mg, $5 \mathrm{~mol} \%$ ), XPhos-Pd-G3 (2.1 mg, $1 \mathrm{~mol} \%)$, KOAc (74 mg, $0.75 \mathrm{mmol}, 3$ equiv) and phenylacetylene $(41 \mu \mathrm{L}, 0.38 \mathrm{mmol}, 1.5$ equiv) were dissolved in dry THF under argon. After stirring at room temperature for 4 hours the reaction was stirred at $85^{\circ} \mathrm{C}$ for 24 hours. The product was isolated through column chromatography ( $n$-hexane/EtOAc $=$ $1: 1)$ as a light yellow solid in $60 \%$ yield $(42.0 \mathrm{mg}) .{ }^{1} \mathbf{H}$ NMR $\left(400 \mathrm{MHz}, \mathrm{CDCl}_{3}\right): \delta 7.58$ $-7.51(\mathrm{~m}, 2 \mathrm{H}), 7.46-7.36(\mathrm{~m}, 4 \mathrm{H}), 6.45(\mathrm{~d}, J=3.2 \mathrm{~Hz}, 1 \mathrm{H}), 6.36(\mathrm{dd}, J=3.3,1.9 \mathrm{~Hz}$, $1 \mathrm{H}), 5.61(\mathrm{~s}, 2 \mathrm{H}), 4.84(\mathrm{~d}, J=6.0 \mathrm{~Hz}, 2 \mathrm{H}), 2.43(\mathrm{t}, J=6.4 \mathrm{~Hz}, 1 \mathrm{H}) ;{ }^{13} \mathbf{C}$ NMR $(101 \mathrm{MHz}$, $\left.\mathrm{CDCl}_{3}\right): \delta 147.5,143.5,131.9,129.9,128.8,121.3,110.9,110.3,102.3,73.1,56.1,45.9$; IR (ATR): 3291, 1564, 1421, 1307, 1244, 1210, 1152, 1116, 1022, 960, 905, 807, 753 $\mathrm{cm}^{-1}$; m.p.: $156-157{ }^{\circ} \mathrm{C}$; HRMS (ESI): calc'd for $\mathrm{C}_{16} \mathrm{H}_{14} \mathrm{~N}_{3} \mathrm{O}_{2}(\mathrm{M}+\mathrm{H})^{+}:$: 280.1086; Found: 280.1086

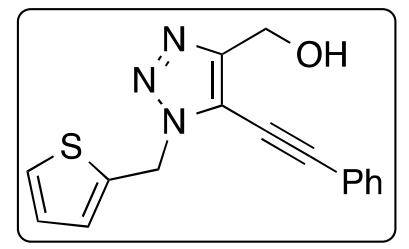

(5-(phenylethynyl)-1-(thiophen-2-ylmethyl)-1H-1,2,3-triazol-4-yl)methanol (9i)

According to general procedure $C$ iodoalkyne $2 \mathrm{a}(45.5 \mathrm{mg}, 0.25 \mathrm{mmol}, 1$ equiv), azide $\mathbf{1 i}$ (33.9 mg, $0.28 \mathrm{mmol}, 1.1$ equiv), CuI (2.3 mg, $5 \mathrm{~mol} \%$ ), TBTA (6.6 mg, $5 \mathrm{~mol} \%$ ), XPhos-Pd-G3 (2.1 mg, $1 \mathrm{~mol} \%)$, KOAc (74 mg, $0.75 \mathrm{mmol}, 3$ equiv) and phenylacetylene $(41 \mu \mathrm{L}, 0.38 \mathrm{mmol}, 1.5$ equiv) were dissolved in dry THF under argon. After stirring at room temperature for 4 hours the reaction was stirred at $85^{\circ} \mathrm{C}$ for 24 hours. The product was isolated through column chromatography $(n$-hexane/EtOAc $=$ $1: 1)$ as a light yellow solid in $66 \%$ yield $(49.0 \mathrm{mg}) .{ }^{1} \mathbf{H}$ NMR $\left(400 \mathrm{MHz}, C D C l_{3}\right): \delta 7.60$ $-7.52(\mathrm{~m}, 2 \mathrm{H}), 7.48-7.36(\mathrm{~m}, 3 \mathrm{H}), 7.29(\mathrm{dd}, J=5.1,1.3 \mathrm{~Hz}, 1 \mathrm{H}), 7.18(\mathrm{dd}, J=3.6,1.1$ $\mathrm{Hz}, 1 \mathrm{H}), 6.98(\mathrm{dd}, J=5.2,3.5 \mathrm{~Hz}, 1 \mathrm{H}), 5.78(\mathrm{~s}, 2 \mathrm{H}), 4.83(\mathrm{~d}, J=5.2 \mathrm{~Hz}, 2 \mathrm{H}), 2.55$ (t, $J=$ $6.7 \mathrm{~Hz}, 1 \mathrm{H}):{ }^{13} \mathrm{C}$ NMR $\left(101 \mathrm{MHz}, C D C l_{3}\right): \delta 149.4,136.2,131.9,130.0,128.8,128.3$, 127.3, 127.3, 127.0, 121.3, 119.3, 102.6, 100.1, 73.3, 56.1, 47.4; IR (ATR): 3289, 1565, 1441, 1430, 1246, 1210, 1154, 1111, 1023, 1000, 959, 931, 859, $805 \mathrm{~cm}^{-1}$; m.p.:108-109 ${ }^{\circ} \mathrm{C}$; HRMS (ESI): calc'd for $\mathrm{C}_{16} \mathrm{H}_{14} \mathrm{~N}_{3} \mathrm{OS}(\mathrm{M}+\mathrm{H})^{+}$: 296.0858 ; Found: 296.0859 .

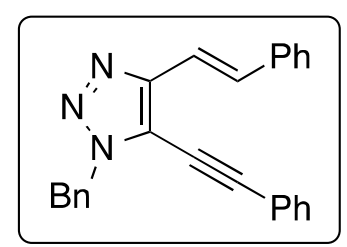

(E)-1-benzyl-5-(phenylethynyl)-4-styryl-1H-1,2,3-triazole (9j)

According to general procedure $\mathrm{C}$ iodoalkyne $\mathbf{2} \mathbf{j}(63.5 \mathrm{mg}, 0.25 \mathrm{mmol}, 1$ equiv), benzyl azide (36.6 mg, $0.28 \mathrm{mmol}, 1.1$ equiv), CuI (2.3 mg, $5 \mathrm{~mol} \%$ ), TBTA (6.6 mg, $5 \mathrm{~mol}$ $\%$ ), XPhos-Pd-G3 (2.1 mg, $1 \mathrm{~mol} \mathrm{\% ),} \mathrm{KOAc} \mathrm{(74} \mathrm{mg,} 0.75 \mathrm{mmol}, 3$ equiv) and phenylacetylene $(41 \mu \mathrm{L}, 0.38 \mathrm{mmol}, 1.5$ equiv) were dissolved in dry THF under argon. After stirring at room temperature for 3 hours the reaction was stirred at $85^{\circ} \mathrm{C}$ for 24 
hours. The product was isolated through column chromatography $(n$-hexane/EtOAc $=$ 6:4) as a light yellow solid in $59 \%$ yield $(53.0 \mathrm{mg}) .{ }^{1} \mathbf{H}$ NMR (500 MHz, $\left.C D C l_{3}\right) \delta 7.68$ $(\mathrm{d}, J=16.5 \mathrm{~Hz}, 1 \mathrm{H}), 7.52(\mathrm{~m}, 4 \mathrm{H}), 7.37(\mathrm{~m}, 10 \mathrm{H}), 7.29(\mathrm{~m}, 1 \mathrm{H}), 7.17(\mathrm{~d}, J=16.9 \mathrm{~Hz}$, $1 \mathrm{H}), 5.64(\mathrm{~s}, 2 \mathrm{H}) ;{ }^{13} \mathbf{C}$ NMR $(126 \mathrm{MHz}, C D C l 3) \delta 147.6,136.7,134.7,131.9,131.5$, $129.7,128.9,128.7,128.5,128.2,128.1,126.7,121.4,117.9,116.2,103.1,74.8,52.9$; IR $\left(\mathrm{NaCl}, \mathrm{CDCl}_{3}\right): 3455,3063,3032,2928,2852,2220,1485,1456,1213,964,754 \mathrm{~cm}^{-1}$; m.p.: 101-102 ${ }^{\circ} \mathrm{C}$; HRMS (ESI): calc'd for $\mathrm{C}_{25} \mathrm{H}_{20} \mathrm{~N}_{3}(\mathrm{M}+\mathrm{H})^{+}$: 362.1657 ; Found: 362.1649
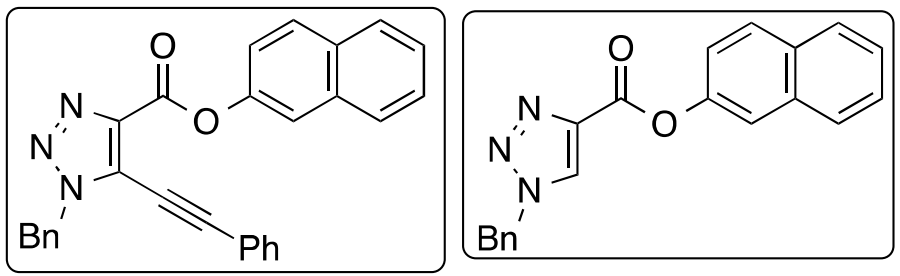

naphthalen-2-yl 1-benzyl-5-(phenylethynyl)-1H-1,2,3-triazole-4-carboxylate (9k) naphthalen-2-yl 1-benzyl-1H-1,2,3-triazole-4-carboxylate (11k)

According to general procedure C iodoalkyne $2 \mathbf{k}(81.1 \mathrm{mg}, 0.25 \mathrm{mmol}, 1$ equiv), benzyl azide (36.6 mg, $0.28 \mathrm{mmol}, 1.1$ equiv), CuI (2.3 mg, $5 \mathrm{~mol} \%$ ), TBTA (6.6 mg, $5 \mathrm{~mol}$ $\%$ ), XPhos-Pd-G3 (2.1 mg, $1 \mathrm{~mol} \%)$, KOAc (74 mg, $0.75 \mathrm{mmol}, 3$ equiv) and phenylacetylene ( $41 \mu \mathrm{L}, 0.38 \mathrm{mmol}, 1.5$ equiv) were dissolved in dry THF under argon. After stirring at room temperature for 1 hour the reaction was stirred at $85^{\circ} \mathrm{C}$ for 18 hours. The crude products was purified by column chromatography $(n$-hexane:EtOAc $=$ $7: 3)$ to afford $9 \mathbf{k}$ as a brown solid in $48 \%$ yield $(41.2 \mathrm{mg})$ and $11 \mathbf{k}$ as a light yellow solid in $39 \%$ yield $(26.3 \mathrm{mg}) .9 \mathrm{k}:{ }^{1} \mathbf{H}$ NMR $\left(400 \mathrm{MHz}, C D C l_{3}\right): \delta 7.86(\mathrm{~m}, 3 \mathrm{H}), 7.75(\mathrm{~d}, J=$ $1.9 \mathrm{~Hz}, 1 \mathrm{H}), 7.43(\mathrm{~m}, 13 \mathrm{H}), 5.73(\mathrm{~s}, 2 \mathrm{H}) ;{ }^{13} \mathbf{C}$ NMR $\left(101 \mathrm{MHz}, C D C l_{3}\right): \delta 158.6,148.0$, $139.8,133.9,133.7,131.9,131.6,130.3,129.5,129.1,128.9,128.6,128.2,127.8,126.6$, 125.8, 125.5, 121.0, 120.6, 118.7, 105.1, 73.4, 53.4; IR (NaCl, $C D C l 3): 3061,3032$, 2228, 1748, 1601, 1449, 1207, 1157, 1032, 756, $689 \mathrm{~cm}^{-1}$; m.p.: 113-114 C; HRMS (ESI): calc'd for $\mathrm{C}_{28} \mathrm{H}_{20} \mathrm{~N}_{3} \mathrm{O}_{2}(\mathrm{M}+\mathrm{H})^{+}: 430.1556$; Found: 430.1553. 11k: ${ }^{1} \mathbf{H}$ NMR (400 $\left.\mathrm{MHz}, \mathrm{CDCl}_{3}\right): \delta 8.15(\mathrm{~s}, 1 \mathrm{H}), 7.93-7.78(\mathrm{~m}, 3 \mathrm{H}), 7.71-7.66(\mathrm{~m}, 1 \mathrm{H}), 7.53-7.45(\mathrm{~m}$, 2H), $7.45-7.40(\mathrm{~m}, 3 \mathrm{H}), 7.37-7.32(\mathrm{~m}, 3 \mathrm{H}), 5.64(\mathrm{~s}, 3 \mathrm{H}) ;{ }^{13} \mathbf{C}$ NMR $(101 \mathrm{MHz}$, $\left.\mathrm{CDCl}_{3}\right): \delta 159.3,148.0,140.0,133.9,133.7,131.8,129.7,129.6,129.5,128.5,128.3$, 127.9, 127.9, 126.8, 126.0, 121.1, 118.9, 54.8; IR (ATR): 3124, 3062, 3042, 1744, 1599, 1464.3, 1363, 1243, 1207, 1180, 1154, 1132, 1046, 1004, 859, 814, $768 \mathrm{~cm}^{-1}$; m.p.: 158$159^{\circ} \mathrm{C}$; HRMS (ESI): calc'd for $\mathrm{C}_{20} \mathrm{H}_{16} \mathrm{~N}_{3} \mathrm{O}_{2}(\mathrm{M}+\mathrm{H})^{+}: 330.1237$; Found: 330.1236 .

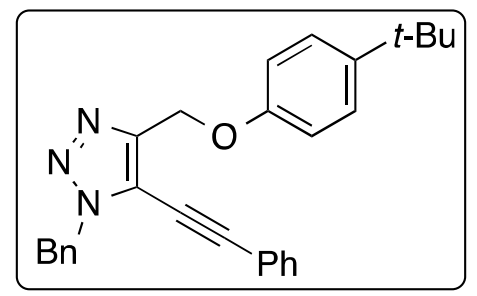

1-benzyl-4-((4-(tert-butyl)phenoxy)methyl)-5-(phenylethynyl)-1H-1,2,3-triazole (9l)

According to general procedure C iodoalkyne 21 ( $78.5 \mathrm{mg}, 0.25 \mathrm{mmol}, 1$ equiv), benzyl azide (36.6 mg, $0.28 \mathrm{mmol}, 1.1$ equiv), CuI (2.3 mg, $5 \mathrm{~mol} \%$ ), TBTA (6.6 mg, $5 \mathrm{~mol}$ $\%$ ), XPhos-Pd-G3 (2.1 mg, $1 \mathrm{~mol} \%)$, KOAc (74 mg, $0.75 \mathrm{mmol}, 3$ equiv) and 
phenylacetylene ( $41 \mu \mathrm{L}, 0.38 \mathrm{mmol}, 1.5$ equiv) were dissolved in dry THF under argon. After stirring at room temperature for 2 hours the reaction was stirred at $85^{\circ} \mathrm{C}$ for 18 hours. The product was isolated through column chromatography $(n$-hexane/EtOAc $=$ 9:1) as an amber solid in $74 \%$ yield (77.9 mg). ${ }^{1} \mathbf{H}$ NMR (500 MHz, $\left.C D C l_{3}\right): \delta 7.45-$ $7.32(\mathrm{~m}, 10 \mathrm{H}), 7.30(\mathrm{~d}, J=9.0 \mathrm{~Hz}, 2 \mathrm{H}), 6.98(\mathrm{~d}, J=8.9 \mathrm{~Hz}, 2 \mathrm{H}), 5.62(\mathrm{~s}, 2 \mathrm{H}), 5.21$ (s, 2H), 1.29 (s, 9H); ${ }^{13} \mathbf{C}$ NMR (126 MHz, $\left.C D C l_{3}\right): \delta 156.2,145.9,143.9,134.5,131.6$, 129.7, 128.9, 128.6, 128.2, 126.2, 121.2, 120.9, 114.5, 102.2, 73.5, 61.4, 53.1, 34.1, 31.5; IR $\left(\mathrm{NaCl}, \mathrm{CDCl}_{3}\right): 3065,3034,2961,2868,1512,1456,1240,1184,1016,829,756 \mathrm{~cm}^{-}$

${ }^{1}$; m.p.: 89-90 ${ }^{\circ} \mathrm{C}$; HRMS (ESI): calc'd for $\mathrm{C}_{28} \mathrm{H}_{28} \mathrm{~N}_{3} \mathrm{O}(\mathrm{M}+\mathrm{H})^{+}$: 422.2232; Found: 422.2235 .

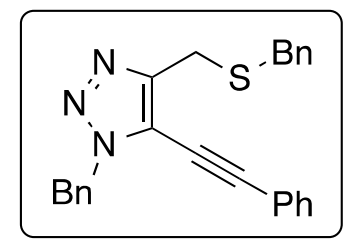

1-benzyl-4-((benzylthio)methyl)-5-(phenylethynyl)-1H-1,2,3-triazole (9m)

According to general procedure $C$ iodoalkyne $2 \mathbf{m}(72.0 \mathrm{mg}, 0.25 \mathrm{mmol}, 1$ equiv), benzy azide (36.6 mg, $0.28 \mathrm{mmol}, 1.1$ equiv), CuI (2.3 mg, $5 \mathrm{~mol} \%$ ), TBTA (6.6 mg, $5 \mathrm{~mol}$ $\%$ ), XPhos-Pd-G3 (2.1 mg, $1 \mathrm{~mol} \mathrm{\% ),} \mathrm{KOAc} \mathrm{(74} \mathrm{mg,} 0.75 \mathrm{mmol}, 3$ equiv) and phenylacetylene $(55 \mu \mathrm{L}, 0.5 \mathrm{mmol}, 2.0$ equiv) were dissolved in dry THF under argon. After stirring at room temperature for 22 hours the reaction was stirred at $85^{\circ} \mathrm{C}$ for 20 hours. The product was isolated through column chromatography ( $n$-hexane/EtOAc $=$ 9:1) as brown oil in 62\% yield (61.0 mg). ${ }^{1} \mathrm{H}$ NMR (400 MHz, $\left.C D C l_{3}\right): \delta 7.48-7.31(\mathrm{~m}$, $12 \mathrm{H}), 7.26(\mathrm{ddt}, J=8.1,6.7,1.9 \mathrm{~Hz}, 2 \mathrm{H}), 7.22-7.17(\mathrm{~m}, 1 \mathrm{H}), 5.59(\mathrm{~s}, 2 \mathrm{H}), 3.78(\mathrm{~s}, 2 \mathrm{H})$, $3.74(\mathrm{~s}, 2 \mathrm{H}) ;{ }^{13} \mathrm{C}$ NMR $\left(101 \mathrm{MHz}, C D C l_{3}\right): \delta 148.4,138.1,134.7,131.7,129.8,129.3$, $129.0,128.7,128.7,128.5,128.3,127.1,121.4,119.5,102.6,73.9,53.2,36.1,25.2$; IR (ATR): 3067, 3031, 2920, 2228, 1699, 1604, 1559, 1495 1454, 1442, 1348, 1323, 1298, 1233, 1204, 1182, 1158, 1112, 1070, 1028, 1000, 916, 825, $754 \mathrm{~cm}^{-1}$; HRMS (ESI): calc'd for $\mathrm{C}_{25} \mathrm{H}_{22} \mathrm{~N}_{3} \mathrm{~S}_{1}(\mathrm{M}+\mathrm{H})^{+}$: 396.1534; Found: 396.1535 .

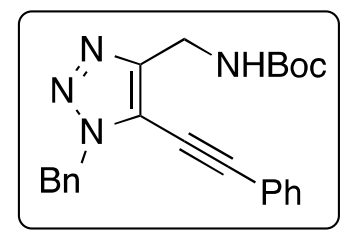

tert-butyl ((1-benzyl-5-(phenylethynyl)-1H-1,2,3-triazol-4-yl)methyl)carbamate (9n)

According to general procedure $C$ iodoalkyne $2 \mathrm{n}$ ( $70.3 \mathrm{mg}, 0.25 \mathrm{mmol}, 1$ equiv), benzyl azide (36.6 mg, $0.28 \mathrm{mmol}, 1.1$ equiv), CuI (2.4 mg, $5 \mathrm{~mol} \%$ ), TBTA (6.6 mg, $5 \mathrm{~mol}$ $\%$ ), XPhos-Pd-G3 (2.1 mg, $1 \mathrm{~mol} \%)$, KOAc (74 mg, $0.6 \mathrm{mmol}, 3$ equiv) and phenylacetylene ( $41 \mu \mathrm{L}, 0.38 \mathrm{mmol}, 1.5$ equiv) were dissolved in dry THF under argon. After stirring at room temperature for 3 hours the reaction was stirred at $85^{\circ} \mathrm{C}$ for 18 hours. The product was isolated through column chromatography $(n$-hexane/EtOAc $=$ $8: 2)$ as a yellow solid in $72 \%$ yield $(70 \mathrm{mg}) .{ }^{1} \mathbf{H}$ NMR $\left(500 \mathrm{MHz}, \mathrm{CDCl}_{3}\right): \delta 7.51-7.45$ $(\mathrm{m}, 2 \mathrm{H}), 7.45-7.29(\mathrm{~m}, 8 \mathrm{H}), 5.60(\mathrm{~s}, 2 \mathrm{H}), 5.14(\mathrm{~s}, 1 \mathrm{H}), 4.52(\mathrm{~d}, J=5.0 \mathrm{~Hz}, 2 \mathrm{H}), 1.42(\mathrm{~s}$, 9H); ${ }^{13}$ C NMR $\left(126 \mathrm{MHz}, C D C l_{3}\right): \delta 155.5,147.2,134.5,131.6,129.7,128.9,128.6$, $128.5,128.2$, 121.2, 119.0, 102.3, 79.6, 73.3, 53.0, 36.2, 28.3; IR (NaCl, $\left.C D C_{3}\right): 3337$, 
2976, 2930, 2359, 2342, 1712, 1506, 1456, 1391, 1366, 1248, 1167, 1047, 1028, 926, 866, 758, $691 \mathrm{~cm}^{-1}$; m.p.: $103-104{ }^{\circ} \mathrm{C}$; HRMS (TOF, ESI+): calc'd for $\mathrm{C}_{23} \mathrm{H}_{25} \mathrm{~N}_{4} \mathrm{O}_{2}$ $(\mathrm{M}+\mathrm{H})^{+}:$398.1972; Found: 389.1980 .

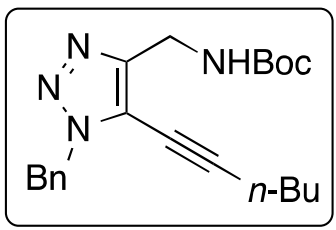

tert-butyl ((1-benzyl-5-(hex-1-yn-1-yl)-1H-1,2,3-triazol-4-yl)methyl)carbamate (90) According to general procedure $C$ iodoalkyne $2 \mathrm{n}(70.3 \mathrm{mg}, 0.25 \mathrm{mmol}, 1$ equiv), benzyl azide (36.6 mg, $0.28 \mathrm{mmol}, 1.1$ equiv), CuI (2.4 mg, $5 \mathrm{~mol} \%$ ), TBTA (6.6 mg, $5 \mathrm{~mol}$ \%), XPhos-Pd-G3 (2.1 mg, $1 \mathrm{~mol} \%$ ), KOAc ( $74 \mathrm{mg}, 0.6 \mathrm{mmol}, 3$ equiv) and 1-hexyne (43 $\mu \mathrm{L}, 0.38 \mathrm{mmol}, 1.5$ equiv) were dissolved in dry THF under argon. After stirring at room temperature for 3 hours the reaction was stirred at $85^{\circ} \mathrm{C}$ for 18 hours. The product was isolated through column chromatography $(n$-hexane/EtOAc $=8: 2)$ as a light yellow oil in $61 \%$ yield $(56 \mathrm{mg}) .{ }^{1} \mathbf{H}$ NMR $\left(500 \mathrm{MHz}, \mathrm{CDCl}_{3}\right): \delta 7.36-7.26(\mathrm{~m}, 5 \mathrm{H}), 5.50(\mathrm{~s}$, 2H), $5.08(\mathrm{~s}, 1 \mathrm{H}), 4.42$ (d, $J=5.0 \mathrm{~Hz}, 2 \mathrm{H}), 2.45$ (t, $J=7.1 \mathrm{~Hz}, 2 \mathrm{H}), 1.60-1.52(\mathrm{~m}, 2 \mathrm{H})$, $1.46-1.36(\mathrm{~m}, 11 \mathrm{H}), 0.92(\mathrm{t}, J=7.3 \mathrm{~Hz}, 3 \mathrm{H}) ;{ }^{13} \mathbf{C}$ NMR $\left(126 \mathrm{MHz}, C C_{C l}\right): \delta 155.7$, $146.4,134.9,128.9,128.5,128.1,119.8,104.7,79.6,65.3,52.8,36.2,30.3,28.5,22.1$, 19.5, 13.7; IR $\left(\mathrm{NaCl}, C D C l_{3}\right): 3347,2961,2932,2872,2342,1717,1497,1456,1366$, 1248, 1171, 1047, 1028, 936, 868, 734, $702 \mathrm{~cm}^{-1}$; m.p.: 52-53 ${ }^{\circ} \mathrm{C}$; HRMS (TOF, ESI+): calc'd for $\mathrm{C}_{21} \mathrm{H}_{29} \mathrm{~N}_{4} \mathrm{O}_{2}(\mathrm{M}+\mathrm{H})^{+}:$369.2285; Found: 369.2291 .

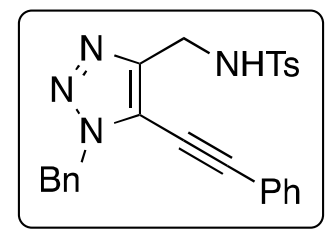

\section{N-((1-benzyl-5-(phenylethynyl)-1H-1,2,3-triazol-4-yl)methyl)-4 methylbenzenesulfonamide (9p)}

According to general procedure C iodoalkyne $2 \mathbf{p}(83.8 \mathrm{mg}, 0.25 \mathrm{mmol}, 1$ equiv), benzyl azide (36.6 mg, $0.28 \mathrm{mmol}, 1.1$ equiv), CuI (2.3 mg, $5 \mathrm{~mol} \%$ ), TBTA (6.6 mg, $5 \mathrm{~mol}$ $\%$ ), XPhos-Pd-G3 (2.1 mg, $1 \mathrm{~mol} \%)$, KOAc (74 mg, $0.75 \mathrm{mmol}, 3$ equiv) and phenylacetylene ( $41 \mu \mathrm{L}, 0.38 \mathrm{mmol}, 1.5$ equiv) were dissolved in dry THF under argon. After stirring at room temperature for 3 hours the reaction was stirred at $85^{\circ} \mathrm{C}$ for 18 hours. The product was isolated through column chromatography $(n$-hexane/EtOAc $=$ $75: 25)$ as a light yellow solid in $87 \%$ yield $(96 \mathrm{mg})$.

The reaction was also conducted on $2 \mathrm{mmol}$ scale. 2p (670 $\mathrm{mg}, 2 \mathrm{mmol})$, benzyl azide (292 mg, $2.2 \mathrm{mmol}, 1.1$ equiv), CuI (20 mg, $5 \mathrm{~mol} \mathrm{\% ),} \mathrm{TBTA} \mathrm{(53} \mathrm{mg,} 5 \mathrm{~mol} \%$ ), XPhosPd-G3 (17 mg $1 \mathrm{~mol} \%$ ), KOAc (593 mg, 3 equiv), and THF (10 mL, 0.2 M). Product was isolated in $81 \%$ yield $(715 \mathrm{mg})$.

The reaction was also conducted using one pot sequential procedure. In an oven-dried microwave vial under an argon atmosphere equipped with a stir bar, $\mathrm{CuI}(2.3 \mathrm{mg}, 5 \mathrm{~mol}$ $\%$ ), TBTA ( $6.6 \mathrm{mg}, 5 \mathrm{~mol} \%$ ) were dissolved in THF $(2.5 \mathrm{~mL})$, followed by the addition of iodoalkyne $2 \mathbf{p}(83.8 \mathrm{mg}, 0.25 \mathrm{mmol}, 1$ equiv), and benzyl azide $(36.6 \mathrm{mg}, 0.28 \mathrm{mmol}$, 1.1 equiv). The reaction was stirred at room temperature for 3 hours followed by the 
addition of XPhos-Pd-G3 (2.1 mg, $1 \mathrm{~mol} \%)$, KOAc (74 mg, $0.75 \mathrm{mmol}, 3$ equiv) and phenylacetylene $\left(41 \mu \mathrm{L}, 0.38 \mathrm{mmol}, 1.5\right.$ equiv) and stirred at $85^{\circ} \mathrm{C}$ for 18 hours. Product was isolated in $86 \%$ yield $(95 \mathrm{mg})$.

${ }^{1}$ H NMR $\left(300 \mathrm{MHz}, C D C l_{3}\right) \delta 7.72(\mathrm{~d}, J=8.3 \mathrm{~Hz}, 2 \mathrm{H}), 7.53-7.28(\mathrm{~m}, 10 \mathrm{H}), 7.19(\mathrm{~d}, J$ $=8.3 \mathrm{~Hz}, 2 \mathrm{H}), 5.52(\mathrm{~s}, 2 \mathrm{H}), 4.34(\mathrm{~d}, J=5.8 \mathrm{~Hz}, 2 \mathrm{H}), 2.36(\mathrm{~s}, 3 \mathrm{H}) ;{ }^{13} \mathbf{C}$ NMR $(126 \mathrm{MHz}$, $\left.\mathrm{CDCl}_{3}\right): \delta 143.5,136.7,134.4,131.8,130.0,129.7,129.1,128.8,128.3,127.3,121.0$, 103.0, 72.9, 53.2, 38.5, 21.6; IR (NaCl, $\left.C D C l_{3}\right): 3472,3314,2923,2853,1331,1157$, 814, 756, 457, 440, $411 \mathrm{~cm}^{-1}$; m.p.: $163-164^{\circ} \mathrm{C}$; HRMS (ESI): calc'd for $\mathrm{C}_{25} \mathrm{H}_{23} \mathrm{~N}_{4} \mathrm{O}_{2} \mathrm{~S}$ $(\mathrm{M}+\mathrm{H})^{+}: 443.1542$; Found: 443.1527

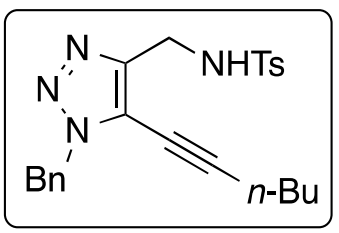

N-((1-benzyl-5-(hex-1-yn-1-yl)-1H-1,2,3-triazol-4-yl)methyl)-4methylbenzenesulfonamide (9q)

According to general procedure $\mathrm{C}$ iodoalkyne $2 \mathbf{p}(83.8 \mathrm{mg}, 0.25 \mathrm{mmol}, 1$ equiv), benzyl azide (36.6 mg, $0.28 \mathrm{mmol}, 1.1$ equiv), CuI (2.4 mg, $5 \mathrm{~mol} \%$ ), TBTA (6.6 mg, $5 \mathrm{~mol}$ \%), XPhos-Pd-G3 (2.1 mg, $1 \mathrm{~mol} \%$ ), KOAc (74 mg, $0.6 \mathrm{mmol}, 3$ equiv) and 1-hexyne (43 $\mu \mathrm{L}, 0.38 \mathrm{mmol}, 1.5$ equiv) were dissolved in dry THF under argon. After stirring at room temperature for 12 hours the reaction was stirred at $85^{\circ} \mathrm{C}$ for 18 hours. The product was isolated through column chromatography ( $n$-hexane/EtOAc $=7: 3)$ as a clear oil in $70 \%$ yield $(74 \mathrm{mg}) .{ }^{1} \mathbf{H}$ NMR $\left(500 \mathrm{MHz}, C D C l_{3}\right): \delta 7.71(\mathrm{~d}, J=8.2 \mathrm{~Hz}, 2 \mathrm{H}), 7.36-7.30$ $(\mathrm{m}, 3 \mathrm{H}), 7.26-7.22(\mathrm{~m}, 2 \mathrm{H}), 7.22-7.18(\mathrm{~m}, 2 \mathrm{H}), 5.42(\mathrm{~s}, 2 \mathrm{H}), 5.03(\mathrm{~s}, 1 \mathrm{H}), 4.25(\mathrm{~d}, J=$ $5.9 \mathrm{~Hz}, 2 \mathrm{H}), 2.46(\mathrm{t}, J=7.1 \mathrm{~Hz}, 2 \mathrm{H}), 2.40-2.35(\mathrm{~m}, 3 \mathrm{H}), 1.61-1.53(\mathrm{~m}, 2 \mathrm{H}), 1.48-$ $1.36(\mathrm{~m}, 2 \mathrm{H}), 0.95(\mathrm{t}, J=7.3 \mathrm{~Hz}, 3 \mathrm{H}) ;{ }^{13} \mathbf{C}$ NMR $\left(126 \mathrm{MHz}, C D C l_{3}\right): \delta 144.4,143.5$, 136.7, 134.6, 129.7, 129.0, 128.7, 128.1, 127.4, 120.2, 105.4, 64.9, 52.8, 38.5, 30.2, 22.1, 21.7, 19.5, 13.7; IR (NaCl, $\left.C D C_{3}\right): 3268,2957,2932,2872,1599,1497,1456,1427$, 1333, 1161, 1094, 1055, 814, 706, $660 \mathrm{~cm}^{-1}$; m.p.: 85-87 ${ }^{\circ} \mathrm{C}$; HRMS (TOF, ESI+): calc'd for $\mathrm{C}_{23} \mathrm{H}_{27} \mathrm{~N}_{4} \mathrm{O}_{2} \mathrm{~S}(\mathrm{M}+\mathrm{H})^{+}$: 423.1849 ; Found: 423.1857 .

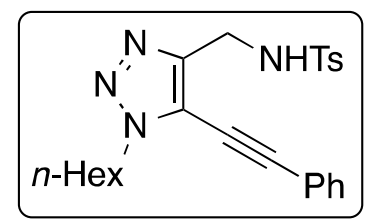

(1-hexyl-5-(phenylethynyl)-1H-1,2,3-triazol-4-yl)methyl)-4-methylbenzenesulfonamide (9r)

According to general procedure $\mathrm{C}$ iodoalkyne $\mathbf{2 p}(83.8 \mathrm{mg}, 0.25 \mathrm{mmol}, 1$ equiv), hexyl azide 1r (35.6 mg, $0.28 \mathrm{mmol}, 1.1$ equiv), CuI (2.3 mg, $5 \mathrm{~mol} \%$ ), TBTA (6.6 mg, $5 \mathrm{~mol}$ $\%$ ), XPhos-Pd-G3 (2.1 mg, $1 \mathrm{~mol} \mathrm{\% ),} \mathrm{KOAc} \mathrm{(74} \mathrm{mg,} 0.75 \mathrm{mmol}, 3$ equiv) and phenylacetylene $(41 \mu \mathrm{L}, 0.38 \mathrm{mmol}, 1.5$ equiv) were dissolved in dry THF under argon. After stirring at room temperature for 2.5 hours the reaction was stirred at $85^{\circ} \mathrm{C}$ for 18 hours. The product was isolated through column chromatography $(n$-hexane/EtOAc $=$ 6:4) as a white solid in $73 \%$ yield $(80.3 \mathrm{mg}) .{ }^{1} \mathbf{H}$ NMR $\left(500 \mathrm{MHz}, C D C l_{3}\right): \delta 7.74(\mathrm{~d}, J=$ $8.2 \mathrm{~Hz}, 2 \mathrm{H}), 7.58-7.52(\mathrm{~m}, 2 \mathrm{H}), 7.47-7.37$ (m, 3H), 7.22 (d, J= 7.9 Hz, 2H), 4.36 (d, $J$ 
$=6.0 \mathrm{~Hz}, 2 \mathrm{H}), 4.35-4.31(\mathrm{t}, J=7.4 \mathrm{~Hz}, 3 \mathrm{H}), 2.37(\mathrm{~s}, 3 \mathrm{H}), 1.89(\mathrm{p}, J=7.2 \mathrm{~Hz}, 2 \mathrm{H}), 1.35$ $-1.28(\mathrm{~m}, 6 \mathrm{H}), 0.87(\mathrm{t}, J=7.1 \mathrm{~Hz}, 3 \mathrm{H}) ;{ }^{13} \mathbf{C}$ NMR $\left(126 \mathrm{MHz}, C D C l_{3}\right): \delta 144.8,143.5$, $136.7,131.8,130.0,129.7,128.8,127.4,121.2,119.6,102.5,72.9,49.4,38.5,31.2,29.9$, 26.22, 22.6, 21.6, 14.1; IR (NaCl, $\left.C D C l_{3}\right): 3198,3032,1558,1437,1332,1161,1093$, 1053, 813, 758, 690, $661 \mathrm{~cm}^{-1}$; m.p.: $127-128^{\circ} \mathrm{C}$; HRMS (ESI+): calc'd for $\mathrm{C}_{24} \mathrm{H}_{29} \mathrm{~N}_{4} \mathrm{O}_{2} \mathrm{~S}$ $(\mathrm{M}+\mathrm{H})^{+}$: 437.2016; Found: 437.2006.

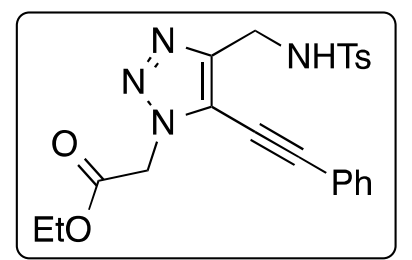

(1-ethylacetate-5-(phenylethynyl)-1H-1,2,3-triazol-4-yl)methyl)-4methylbenzenesulfonamide (9s)

According to general procedure C iodoalkyne $2 \mathbf{p}(83.8 \mathrm{mg}, 0.25 \mathrm{mmol}, 1$ equiv), azide $1 \mathrm{~s}$ (36.2 mg, $0.28 \mathrm{mmol}, 1.1$ equiv), CuI (2.3 mg, $5 \mathrm{~mol} \%$ ), TBTA (6.6 mg, $5 \mathrm{~mol} \%$ ), XPhos-Pd-G3 (2.1 mg, $1 \mathrm{~mol} \%$ ), KOAc (74 mg, $0.75 \mathrm{mmol}, 3$ equiv) and phenylacetylene ( $41 \mu \mathrm{L}, 0.38 \mathrm{mmol}, 1.5$ equiv) were dissolved in dry THF under argon. After stirring at room temperature for 5 hours the reaction was stirred at $85^{\circ} \mathrm{C}$ for 15 hours. The product was isolated through column chromatography $(n$-hexane/EtOAc $=$ $6: 4)$ as a white solid in $77 \%$ yield $(84.4 \mathrm{mg}) .{ }^{1} \mathbf{H}$ NMR $\left(400 \mathrm{MHz}, C D C l_{3}\right): \delta 7.76(\mathrm{~d}, J=$ $8.3 \mathrm{~Hz}, 2 \mathrm{H}), 7.56-7.49(\mathrm{~m}, 2 \mathrm{H}), 7.47-7.36(\mathrm{~m}, 3 \mathrm{H}), 7.25$ (d, $J=8.7 \mathrm{~Hz}, 2 \mathrm{H}), 5.12$ (s, $2 \mathrm{H}), 4.37$ (d, $J=5.9 \mathrm{~Hz}, 2 \mathrm{H}), 4.26(\mathrm{q}, J=7.1 \mathrm{~Hz}, 2 \mathrm{H}), 2.39(\mathrm{~s}, 3 \mathrm{H}), 1.27(\mathrm{t}, J=7.1 \mathrm{~Hz}$, $3 \mathrm{H}) ;{ }^{13} \mathrm{C}$ NMR $\left(126 \mathrm{MHz}, \mathrm{CDCl}_{3}\right): \delta 165.6,144.9,143.7,136.6,131.9,130.2,129.8$, $128.8,127.4,120.8,103.4,72.2,62.7,50.0,38.6,21.7,14.2 ; \mathbf{I R}\left(\mathrm{NaCl}, \mathrm{CDCl}_{3}\right): 3265$, 2341, 2332, 1751, 1373, 1332, 1213, 1161, 1093, 688, $655 \mathrm{~cm}^{-1}$; m.p.: 126-126.5 ${ }^{\circ}$; HRMS (ESI+): calc'd for $\mathrm{C}_{22} \mathrm{H}_{23} \mathrm{~N}_{4} \mathrm{O}_{4} \mathrm{~S}(\mathrm{M}+\mathrm{H})^{+}$: 439.1429; Found: 439.1435 .
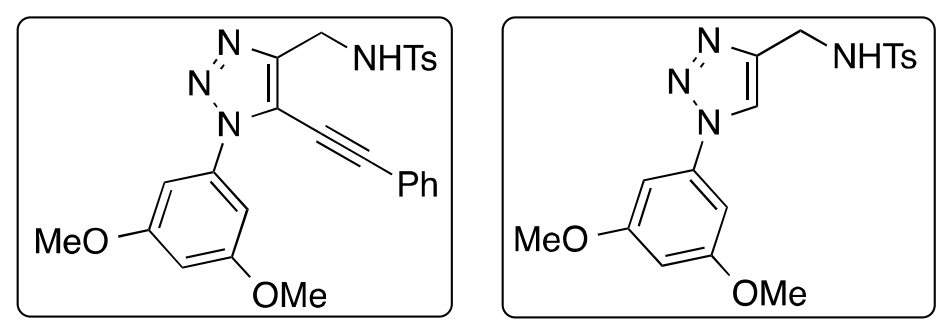

N-((1-(3,5-dimethoxyphenyl)-5-(phenylethynyl)-1H-1,2,3-triazol-4-yl)methyl)-4methylbenzenesulfonamide (9t)

N-((1-(3,5-dimethoxyphenyl)-1H-1,2,3-triazol-4-yl)methyl)-4-

methylbenzenesulfonamide (11t)

According to general procedure C iodoalkyne $2 \mathbf{p}(67 \mathrm{mg}, 0.2 \mathrm{mmol}, 1$ equiv), azide $\mathbf{1 t}$ (39.4 mg, $0.22 \mathrm{mmol}, 1.1$ equiv), CuI (1.9 mg, $5 \mathrm{~mol} \%$ ), TBTA (5.3 mg, $5 \mathrm{~mol} \%$ ), XPhos-Pd-G3 (1.7 mg, $1 \mathrm{~mol} \%$ ), KOAc (59 mg, $0.6 \mathrm{mmol}, 3$ equiv) and phenylacetylene (33 $\mu \mathrm{L}, 0.3 \mathrm{mmol}, 1.5$ equiv) were dissolved in dry THF and added $4 \square$ molecular sieves (bead) under argon. After stirring at room temperature for 8 hours the reaction was stirred at $85^{\circ} \mathrm{C}$ for 18 hours. The crude products was purified by column chromatography ( $n$ - 
hexane:EtOAc $=7: 3)$ to afforded $9 \mathrm{t}$ as a brown oil in $52 \%$ yield $(51 \mathrm{mg})$ and $\mathbf{1 1 t}$ as a light yellow solid in $27 \%$ yield $(21 \mathrm{mg}) .9 \mathrm{9t} ;{ }^{1} \mathbf{H}$ NMR $\left(500 \mathrm{MHz}, C D C l_{3}\right): \delta 7.73(\mathrm{~d}, J=$ $8.3 \mathrm{~Hz}, 2 \mathrm{H}), 7.51(\mathrm{~d}, J=6.4 \mathrm{~Hz}, 2 \mathrm{H}), 7.46-7.36(\mathrm{~m}, 3 \mathrm{H}), 7.18(\mathrm{~d}, J=7.9 \mathrm{~Hz}, 2 \mathrm{H}), 6.90$ $(\mathrm{d}, J=2.3 \mathrm{~Hz}, 2 \mathrm{H}), 6.55(\mathrm{~s}, 1 \mathrm{H}), 4.45(\mathrm{~d}, J=6.2 \mathrm{~Hz}, 2 \mathrm{H}), 3.82(\mathrm{~s}, 6 \mathrm{H}), 2.31(\mathrm{~s}, 3 \mathrm{H}) ;{ }^{13} \mathrm{C}$ NMR $\left(126 \mathrm{MHz}, C D C l_{3}\right): \delta 161.2,146.0,143.5,137.7,136.9,131.7,130.0,129.6$, $128.8,127.4,121.0,119.3,103.0,101.6,101.5,73.9,55.8,38.3,21.5$; IR $(\mathrm{NaCl}$, $\left.\mathrm{CDCl}_{3},\right): 3265,2939,2359,2224,1614,1599,1456,1487,1429,1330,1278,1207$, $1159,1062,910,813,758,731,688 \mathrm{~cm}^{-1}$; HRMS (DART): calc'd for $\mathrm{C}_{26} \mathrm{H}_{25} \mathrm{~N}_{4} \mathrm{O}_{4} \mathrm{~S}(\mathrm{M}$ $+\mathrm{H})^{+}: 489.15806$; Found: 489.15965 .

11t; ${ }^{1} \mathbf{H}$ NMR $\left(400 \mathrm{MHz}, C D C l_{3}\right) \delta 7.77-7.72(\mathrm{~m}, 2 \mathrm{H}), 7.69(\mathrm{~s}, 1 \mathrm{H}), 7.29(\mathrm{dt}, J=7.9$, $0.7 \mathrm{~Hz}, 2 \mathrm{H}), 6.78(\mathrm{~d}, J=2.2 \mathrm{~Hz}, 2 \mathrm{H}), 6.50(\mathrm{t}, J=2.2 \mathrm{~Hz}, 1 \mathrm{H}), 5.02(\mathrm{t}, J=6.3 \mathrm{~Hz}, 1 \mathrm{H})$, $4.36(\mathrm{~d}, J=0.7 \mathrm{~Hz}, 2 \mathrm{H}), 3.85(\mathrm{~s}, 6 \mathrm{H}), 2.39(\mathrm{~s}, 3 \mathrm{H}) .{ }^{13} \mathrm{C} \mathrm{NMR}\left(100 \mathrm{MHz}, C D C l_{3}\right) \delta 161.6$, $144.5,143.9,138.3,136.9,129.9,127.4,120.7,100.8,99.0,55.9,38.8,21.6$; IR (ATR): 3076, 2950, 2869,1612, 1598, 1500, 1464, 1440, 1429, 1288, 1212, 1158, 1093, 1035 , 1014, 860, $820 \mathrm{~cm}^{-1}$; m.p.: $132-134{ }^{\circ} \mathrm{C}$; HRMS (ESI): calc'd for $\mathrm{C}_{18} \mathrm{H}_{21} \mathrm{~N}_{4} \mathrm{O}_{4} \mathrm{~S}(\mathrm{M}+\mathrm{H})^{+}$: 389.1278; Found: 389.1268

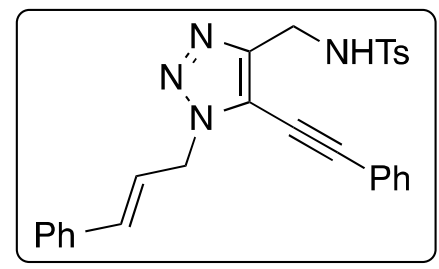

N-((1-cinnamyl-5-(phenylethynyl)-1H-1,2,3-triazol-4-yl)methyl)-4methylbenzenesulfonamide $(9 \mathrm{u})$

According to general procedure $\mathrm{C}$ iodoalkyne $\mathbf{2 p}(83.8 \mathrm{mg}, 0.25 \mathrm{mmol}, 1$ equiv), azide 1 u (44.6 mg, $0.28 \mathrm{mmol}, 1.1$ equiv), CuI (2.3 mg, $5 \mathrm{~mol} \%$ ), TBTA (6.6 mg, $5 \mathrm{~mol} \%$ ), XPhos-Pd-G3 (2.1 mg, $1 \mathrm{~mol} \%)$, KOAc (74 mg, $0.75 \mathrm{mmol}, 3$ equiv) and phenylacetylene $(41 \mu \mathrm{L}, 0.38 \mathrm{mmol}, 1.5$ equiv) were dissolved in dry THF under argon. After stirring at room temperature for 5 hours the reaction was stirred at $85^{\circ} \mathrm{C}$ for 19 hours. The product was isolated through column chromatography $(n$-hexane/EtOAc $=$ $6: 4)$ as a white solid in $78 \%$ yield $(90.8 \mathrm{mg}) .{ }^{1} \mathbf{H}$ NMR $\left(400 \mathrm{MHz}, C D C l_{3}\right): \delta 7.74(\mathrm{~d}, J=$ $8.3 \mathrm{~Hz}, 2 \mathrm{H}), 7.56-7.49(\mathrm{~m}, 2 \mathrm{H}), 7.47-7.27(\mathrm{~m}, 8 \mathrm{H}), 7.21(\mathrm{~d}, J=7.6 \mathrm{~Hz}, 2 \mathrm{H}), 6.68(\mathrm{~d}, J$ $=15.8 \mathrm{~Hz}, 1 \mathrm{H}), 6.31(\mathrm{dt}, J=15.8,6.6 \mathrm{~Hz}, 1 \mathrm{H}), 5.12(\mathrm{~d}, J=6.6 \mathrm{~Hz}, 2 \mathrm{H}), 4.37(\mathrm{~d}, J=6.0$ $\mathrm{Hz}, 2 \mathrm{H}), 2.33$ (s, 3H); ${ }^{13} \mathbf{C}$ NMR (126 MHz, $\left.C D C l_{3}\right): \delta 145.2,143.6,136.7,135.7,131.9$, 130.1, 129.7, 128.84, 128.81, 128.6, 127.4, 126.8, 121.5, 121.1, 119.8, 103.1, 72.8, 51.6, 38.5, 21.6; IR $\left(\mathrm{NaCl}, \mathrm{CDCl}_{3}\right): 3277,2359,1751,1332,1213,1161,1093,758,688,655$ $\mathrm{cm}^{-1}$; m.p.: $124.1-125.1^{\circ} \mathrm{C}$; HRMS (ESI+): calc'd for $\mathrm{C}_{27} \mathrm{H}_{25} \mathrm{~N}_{4} \mathrm{O}_{2} \mathrm{~S}(\mathrm{M}+\mathrm{H})^{+}: 469.1684$; Found: 469.1693.

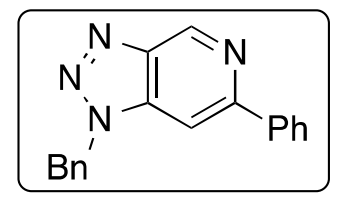

1-benzyl-6-phenyl-1H-[1,2,3]triazolo[4,5-c]pyridine (12a)

According to general procedure D, 9p $\left(88.5 \mathrm{mg}, 0.2 \mathrm{mmol}, 1\right.$ equiv) and $\mathrm{Cs}_{2} \mathrm{CO}_{3}$ (163 $\mathrm{mg}, 0.5 \mathrm{mmol}, 2.5$ equiv) were dissolved in dioxane $(2 \mathrm{~mL})$ in a 2-dram vial equipped 
with a stir bar and sealed with a telfon coated cap and stirred at $120{ }^{\circ} \mathrm{C}$ for $24 \mathrm{~h}$. The mixture was cooled to r.t. and $\mathrm{KOt}$ - $\mathrm{Bu}(22 \mathrm{mg}, 0.2 \mathrm{mmol}, 1$ equiv) was added followed by stirring at r.t. for an additional $8 \mathrm{~h}$. Subsequent work up and isolation using column chromatography ( $n$-hexane:EtOAc 9:1) afforded the product as a colorless solid in $88 \%$ yield (50.4 mg). ${ }^{1} \mathbf{H}$ NMR (400 MHz, $\left.C D C l_{3}\right): \delta 9.52(\mathrm{~s}, 1 \mathrm{H}), 8.01-7.89(\mathrm{~m}, 2 \mathrm{H}), 7.56$ $(\mathrm{d}, J=0.9 \mathrm{~Hz}, 1 \mathrm{H}), 7.52-7.29(\mathrm{~m}, 8 \mathrm{H}), 5.89(\mathrm{~s}, 2 \mathrm{H}) ;{ }^{13} \mathbf{C}$ NMR $\left(101 \mathrm{MHz}, C D C l_{3}\right): \delta$ 154.1, 144.3, 143.1, 139.0, 137.8, 134.1, 129.38, 129.36, 129.0, 127.9, 127.6, 100.5, 52.5; IR (NaCl, $\left.C D C l_{3}\right): 2359,2342,1611,1570,1497,1452,1260,1209,1119,1059$, 1017, 951, 914, 854, 783, 760, 712, $692 \mathrm{~cm}^{-1}$; m.p.: 114-115 ${ }^{\circ} \mathrm{C}$; HRMS (TOF, DART+): calc'd for $\mathrm{C}_{18} \mathrm{H}_{15} \mathrm{~N}_{4}(\mathrm{M}+\mathrm{H})^{+}$: 287.12967; Found: 287.12987.

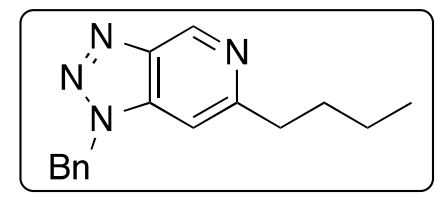

1-benzyl-6-butyl-1H-[1,2,3]triazolo[4,5-c]pyridine (12b)

According to general procedure $\mathrm{D}, \mathbf{9 q}\left(84.5 \mathrm{mg}, 0.2 \mathrm{mmol}, 1\right.$ equiv) and $\mathrm{Cs}_{2} \mathrm{CO}_{3}(163$ $\mathrm{mg}, 0.5 \mathrm{mmol}, 2.5$ equiv) were dissolved in dioxane $(2 \mathrm{~mL})$ in a 2-dram vial equipped with a stir bar and sealed with a teflon coated cap and stirred at $120{ }^{\circ} \mathrm{C}$ for $24 \mathrm{~h}$. The mixture was cooled to r.t. and $\mathrm{KO} t$ - $\mathrm{Bu}$ ( $22 \mathrm{mg}, 0.2 \mathrm{mmol}, 1$ equiv) was added followed by stirring at r.t. for an additional $8 \mathrm{~h}$. Subsequent work up and isolation using column chromatography ( $n$-hexane:EtOAc 8:2) afforded the product as clear oil in $52 \%$ yield $(27.7 \mathrm{mg}) .{ }^{1} \mathbf{H}$ NMR $\left(400 \mathrm{MHz}, \mathrm{CDCl}_{3}\right): \delta 9.37(\mathrm{~s}, 1 \mathrm{H}), 7.43-7.27(\mathrm{~m}, 5 \mathrm{H}), 7.03(\mathrm{~s}$, $1 \mathrm{H}), 5.82(\mathrm{~d}, J=5.7 \mathrm{~Hz}, 2 \mathrm{H}), 2.87(\mathrm{t}, J=7.8 \mathrm{~Hz}, 2 \mathrm{H}), 1.80-1.62(\mathrm{~m}, 2 \mathrm{H}), 1.36(\mathrm{p}, J=$ $7.4 \mathrm{~Hz}, 2 \mathrm{H}), 0.92(\mathrm{t}, J=7.4 \mathrm{~Hz}, 3 \mathrm{H}) ;{ }^{13} \mathbf{C}$ NMR $\left(101 \mathrm{MHz}, C D C l_{3}\right): \delta 158.8,144.1$, 142.6, 137.4, 134.3, 129.3, 128.9, 127.8, 102.1, 52.3, 38.3, 32.2, 22.5, 14.0; IR ( NaCl, $\mathrm{CDCl}_{3}$ ): 2956, 2923, 2870, 2858, 1610, 1577, 1496, 1456, 1437, 1213, 1085, 1057, 958, 717, $696 \mathrm{~cm}^{-1}$; HRMS (DART): calc'd for $\mathrm{C}_{16} \mathrm{H}_{19} \mathrm{~N}_{4}(\mathrm{M}+\mathrm{H})^{+}$: 267.16097; Found: 267.16159 .

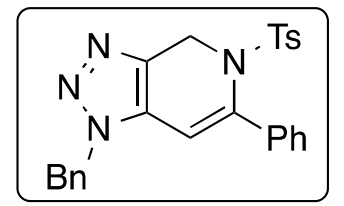

1-benzyl-6-phenyl-5-tosyl-4,5-dihydro-1H-[1,2,3]triazolo[4,5-c/pyridine (12a')

According to general procedure $\mathrm{D}, 7 \mathrm{l}\left(88.5 \mathrm{mg}, 0.2 \mathrm{mmol}, 1\right.$ equiv) and $\mathrm{Cs}_{2} \mathrm{CO}_{3}(163 \mathrm{mg}$, $0.5 \mathrm{mmol}, 2.5$ equiv) were dissolved in dioxane $(2 \mathrm{~mL})$ in a 2 dram vial equipped with a stir bar and sealed with a telfon coated cap and stirred at $120{ }^{\circ} \mathrm{C}$ for $24 \mathrm{~h}$. Subsequent work up and isolation using column chromatography ( $n$-hexane:EtOAc 7:3) as a colorless solid. ${ }^{1} \mathbf{H}$ NMR (500 MHz, $\left.C D C l_{3}\right): \delta 7.55-7.50(\mathrm{~m}, 2 \mathrm{H}), 7.41-7.36(\mathrm{~m}, 6 \mathrm{H}), 7.35(\mathrm{~d}, J$ $=8.3 \mathrm{~Hz}, 2 \mathrm{H}), 7.13-7.08(\mathrm{~m}, 2 \mathrm{H}), 6.99(\mathrm{dd}, J=8.6,0.7 \mathrm{~Hz}, 2 \mathrm{H}), 6.07(\mathrm{~s}, 1 \mathrm{H}), 5.21(\mathrm{~s}$, $2 \mathrm{H}), 5.14$ (s, 2H), 2.31 (s, 3H); ${ }^{13}$ C NMR (126 MHz, $\left.C D C l_{3}\right): \delta 143.8,142.5,139.6$, $137.4,134.5,133.9,130.3,129.8,129.3,129.0,128.9,128.6,128.1,127.54,127.51$, 105.8, 52.6, 45.5, 21.7; IR (NaCl, $\left.C D C l_{3}\right): 3063,3032,2359,2332,1597,1456,1356$, 1240, 1165, 1088, 1028, 912, $814 \mathrm{~cm}^{-1}$; m.p.: $161-162{ }^{\circ} \mathrm{C}$; HRMS (TOF, DART + ): calc'd for $\mathrm{C}_{25} \mathrm{H}_{23} \mathrm{~N}_{4} \mathrm{O}_{2} \mathrm{~S}(\mathrm{M}+\mathrm{H})^{+}$: 443.15417; Found: 443.15507 . 


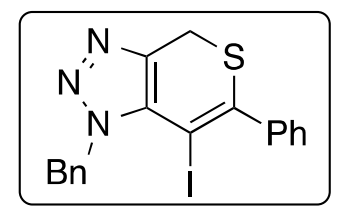

1-benzyl-7-iodo-6-phenyl-1,4-dihydrothiopyrano[3,4-d][1,2,3]triazole (13)

To a solution of $9 \mathrm{~m}(39.5 \mathrm{mg}, 0.1 \mathrm{mmol})$ in $\mathrm{CH}_{2} \mathrm{Cl}_{2}(2 \mathrm{ml})$ was added $\mathrm{K}_{2} \mathrm{CO}_{3}(30.4 \mathrm{mg}$, $0.12 \mathrm{mmol}, 1.2$ equiv) at $0{ }^{\circ} \mathrm{C}$ under an argon atmosphere. To the mixture was added a solution of $\mathrm{I}_{2}\left(27.6 \mathrm{mg}, 0.2 \mathrm{mmol}, 2\right.$ equiv) in $\mathrm{CH}_{2} \mathrm{Cl}_{2}(0.2 \mathrm{ml})$ at the same temperature. Resultant solution was stirred for $24 \mathrm{~h}$ at $40{ }^{\circ} \mathrm{C}$. After cooled to room temperature, The reaction mixture was diluted with $\mathrm{CH}_{2} \mathrm{Cl}_{2}$ and washed with aqueous $\mathrm{Na}_{2} \mathrm{~S}_{2} \mathrm{O}_{3}$ solution, brine and then dried over $\mathrm{Na}_{2} \mathrm{SO}_{4}$, filtered concentrated under reduced pressure to afford a crude mixture. The crude mixture was purified by flash column chromatography with $n$ hexane/EtOAc (8:2) afforded the product as a light yellow solid (20 mg, 46\%). ${ }^{1} \mathbf{H}$ NMR $\left(500 \mathrm{MHz}, C D C l_{3}\right): \delta 7.43-7.27(\mathrm{~m}, 8 \mathrm{H}), 7.20-7.14(\mathrm{~m}, 2 \mathrm{H}), 5.99(\mathrm{~s}, 2 \mathrm{H}), 4.27(\mathrm{~s}$, $2 \mathrm{H}) ;{ }^{13} \mathrm{C}$ NMR $\left(101 \mathrm{MHz}, C D C l_{3}\right): \delta 145.2,139.8,139.2,135.6,133.6,130.6,129.8$, 128.8, 128.4, 128.1, 127.1, 65.1, 52.5, 26.3; IR (ATR):3061, 3037, 2911, 1547, 1454, 1416, 1349, 1274, 1204, 1108, 1097, 1071, 1029, 935, 893, $830 \mathrm{~cm}^{-1}$; m.p.: $179{ }^{\circ} \mathrm{C}$ (decomposition); HRMS (ESI): calc'd for $\mathrm{C}_{18} \mathrm{H}_{15} \mathrm{IN}_{3} \mathrm{~S}(\mathrm{M}+\mathrm{H})^{+}$: 432.0031; Found: 432.0030 . 


\section{X-ray Crystallographic Data}

1-benzyl-6-phenyl-5-tosyl-4,5-dihydro-1H-[1,2,3]triazolo[4,5-c/pyridine (12a')

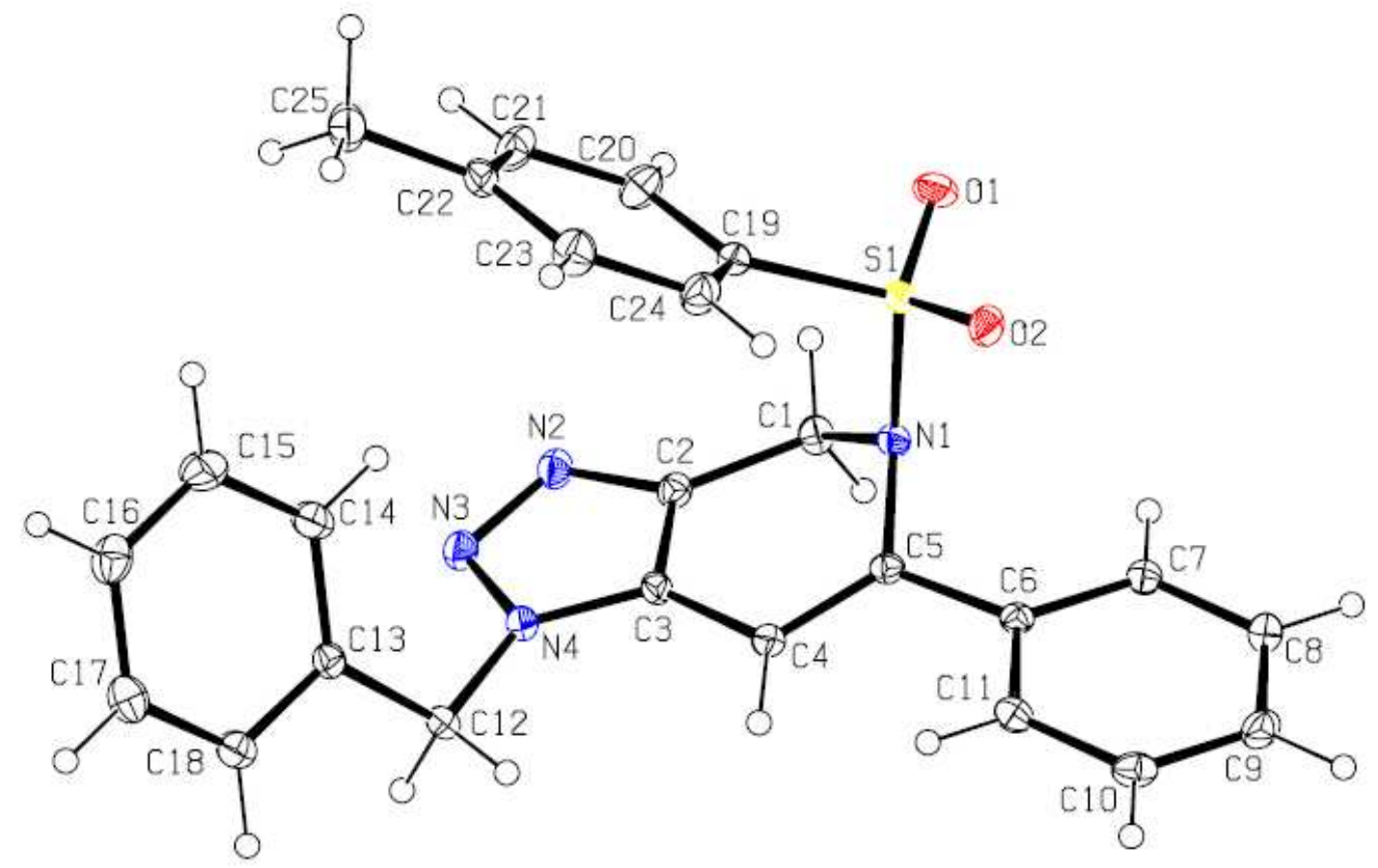

Identification code

Empirical formula

Formula weight

Temperature

Wavelength

Crystal system

Space group

Unit cell dimensions

Volume

Z

Density (calculated)

Absorption coefficient

$\mathrm{F}(000)$

Crystal size d1528

C25 H22 N4 O2 S

442.52

147(2) K

$1.54178 \AA$

Orthorhombic

Pbca

$\begin{array}{ll}\mathrm{a}=19.1187(5) \AA & \mathrm{a}=90^{\circ} . \\ \mathrm{b}=10.2219(2) \AA & \mathrm{b}=90^{\circ} . \\ \mathrm{c}=21.9587(5) \AA & \mathrm{g}=90^{\circ} .\end{array}$

4291.38(17) $\AA^{3}$

8

$1.370 \mathrm{Mg} / \mathrm{m}^{3}$

$1.591 \mathrm{~mm}^{-1}$

1856

$0.210 \times 0.200 \times 0.170 \mathrm{~mm}^{3}$ 
Theta range for data collection

Index ranges

Reflections collected

Independent reflections

Completeness to theta $=67.071^{\circ}$

Absorption correction

Max. and min. transmission

Refinement method

Data / restraints / parameters

Goodness-of-fit on $\mathrm{F}^{2}$

Final $\mathrm{R}$ indices [I $>2 \operatorname{sigma}(\mathrm{I})]$

$\mathrm{R}$ indices (all data)

Extinction coefficient

Largest diff. peak and hole
4.625 to $67.071^{\circ}$.

$-22<=\mathrm{h}<=22,-12<=\mathrm{k}<=12,-25<=1<=26$

77253

$3800[\mathrm{R}(\mathrm{int})=0.0340]$

$99.3 \%$

Semi-empirical from equivalents

0.7529 and 0.6870

Full-matrix least-squares on $\mathrm{F}^{2}$

$3800 / 0$ / 290

1.013

$\mathrm{R} 1=0.0311, \mathrm{wR} 2=0.0809$

$\mathrm{R} 1=0.0323, \mathrm{wR} 2=0.0821$

$\mathrm{n} / \mathrm{a}$

0.217 and -0.481 e. $\AA^{-3}$ 


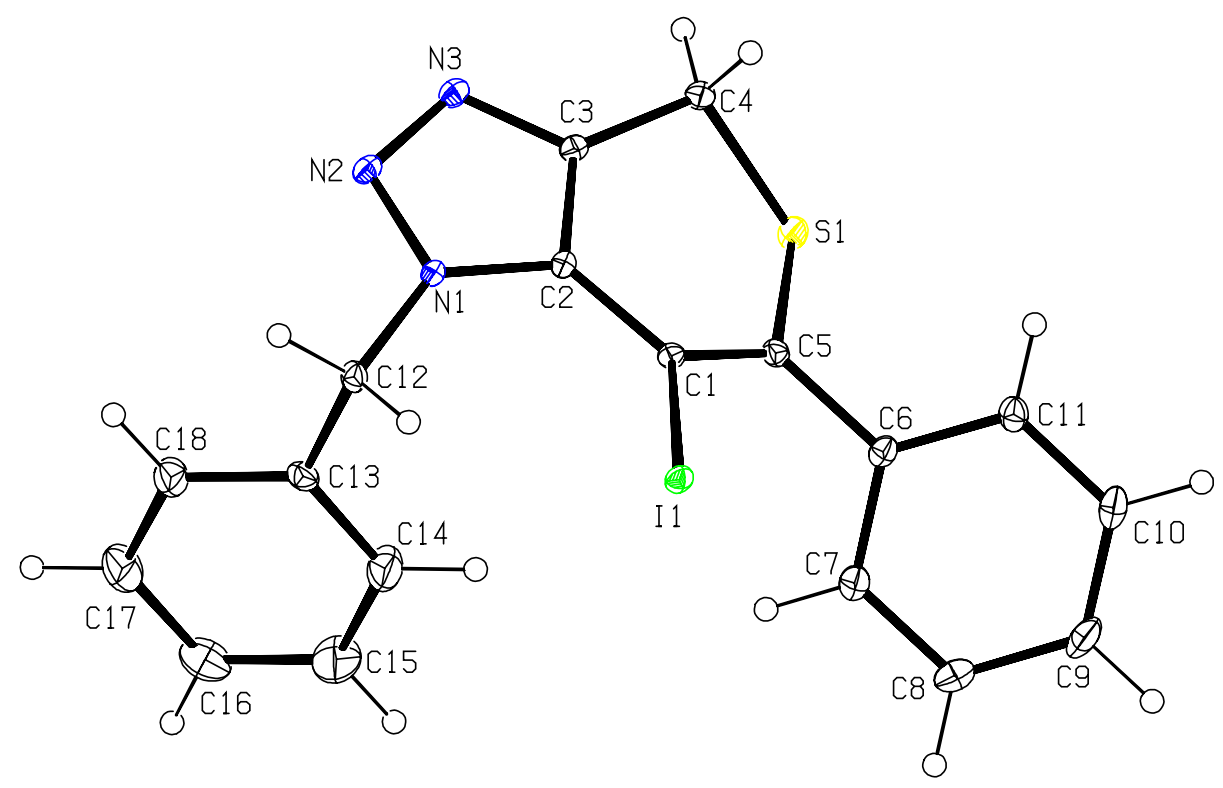

Identification code

Empirical formula

Formula weight

Temperature

Wavelength

Crystal system

Space group

Unit cell dimensions

Volume

Z

Density (calculated)

Absorption coefficient

$\mathrm{F}(000)$

Crystal size

Theta range for data collection

Index ranges

Reflections collected

Independent reflections

Completeness to theta $=25.242^{\circ}$ d15128

C18 H14 I N3 S

431.28

147(2) K

$0.71073 \AA$

Triclinic

P-1

$\mathrm{a}=8.0454(5) \AA$ $\mathrm{a}=91.647(2)^{\circ}$.

$\mathrm{b}=9.1035(5) \AA$ $\mathrm{b}=96.901(2)^{\circ}$.

$\mathrm{c}=11.6624(8) \AA$ $\mathrm{g}=93.582(2)^{\circ}$. 845.76(9) $\AA^{3}$

2

$1.694 \mathrm{Mg} / \mathrm{m}^{3}$

$2.019 \mathrm{~mm}^{-1}$

424

$0.100 \times 0.040 \times 0.020 \mathrm{~mm}^{3}$

1.760 to $27.571^{\circ}$.

$-10<=\mathrm{h}<=10,-11<=\mathrm{k}<=11,-15<=\mathrm{l}<=15$

27541

$3912[\mathrm{R}(\mathrm{int})=0.0281]$

$100.0 \%$ 
Absorption correction

Max. and min. transmission

Refinement method

Data / restraints / parameters

Goodness-of-fit on $\mathrm{F}^{2}$

Final $R$ indices [I $>2 \operatorname{sigma}(\mathrm{I})]$

$\mathrm{R}$ indices (all data)

Extinction coefficient

Largest diff. peak and hole
Semi-empirical from equivalents

0.7456 and 0.6828

Full-matrix least-squares on $\mathrm{F}^{2}$

3912 / 0 / 208

1.025

$\mathrm{R} 1=0.0153, \mathrm{wR} 2=0.0324$

$\mathrm{R} 1=0.0195, \mathrm{wR} 2=0.0335$

$\mathrm{n} / \mathrm{a}$

0.368 and -0.274 e. $\AA^{-3}$ 


\section{NMR Spectroscopic Data}
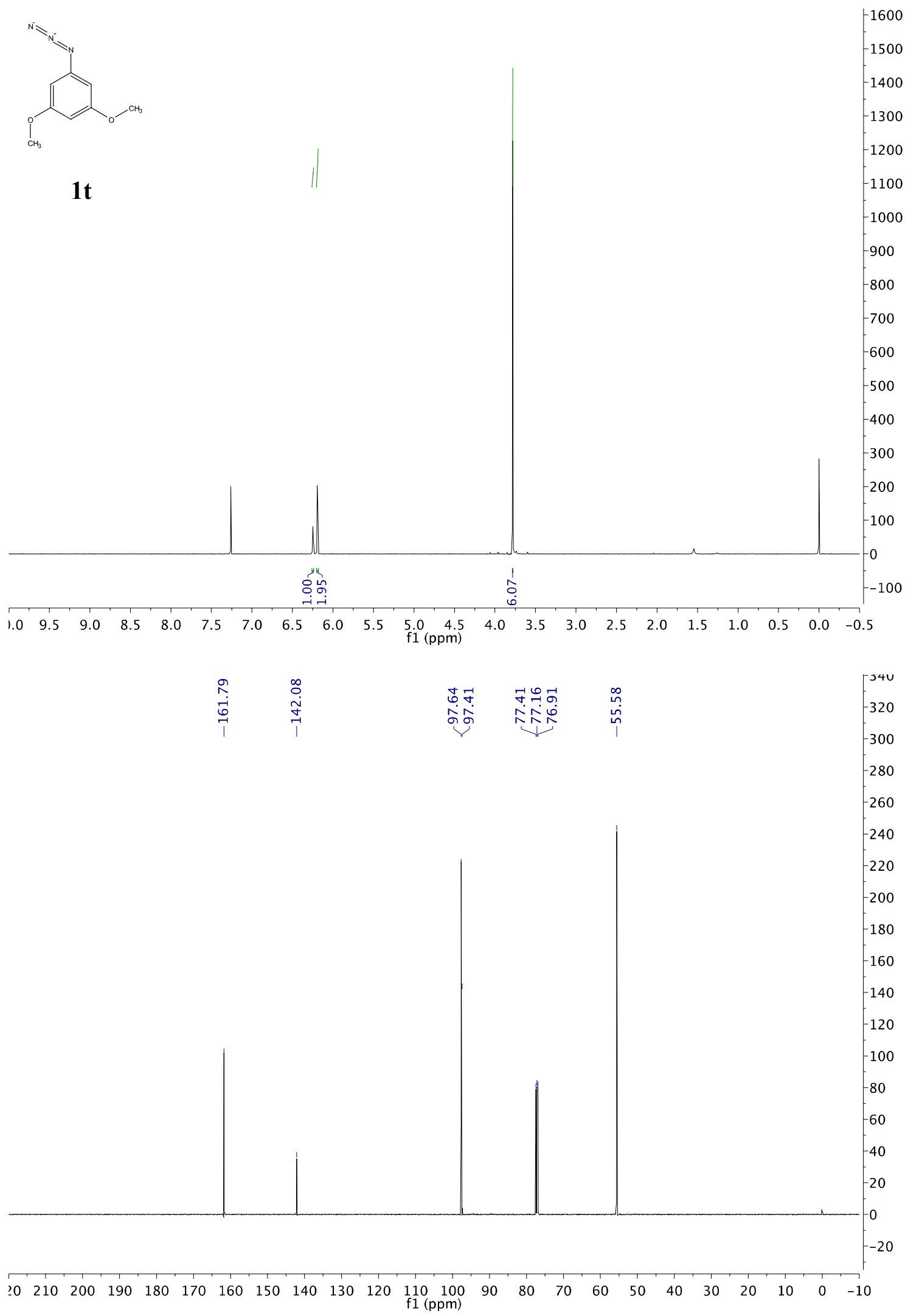

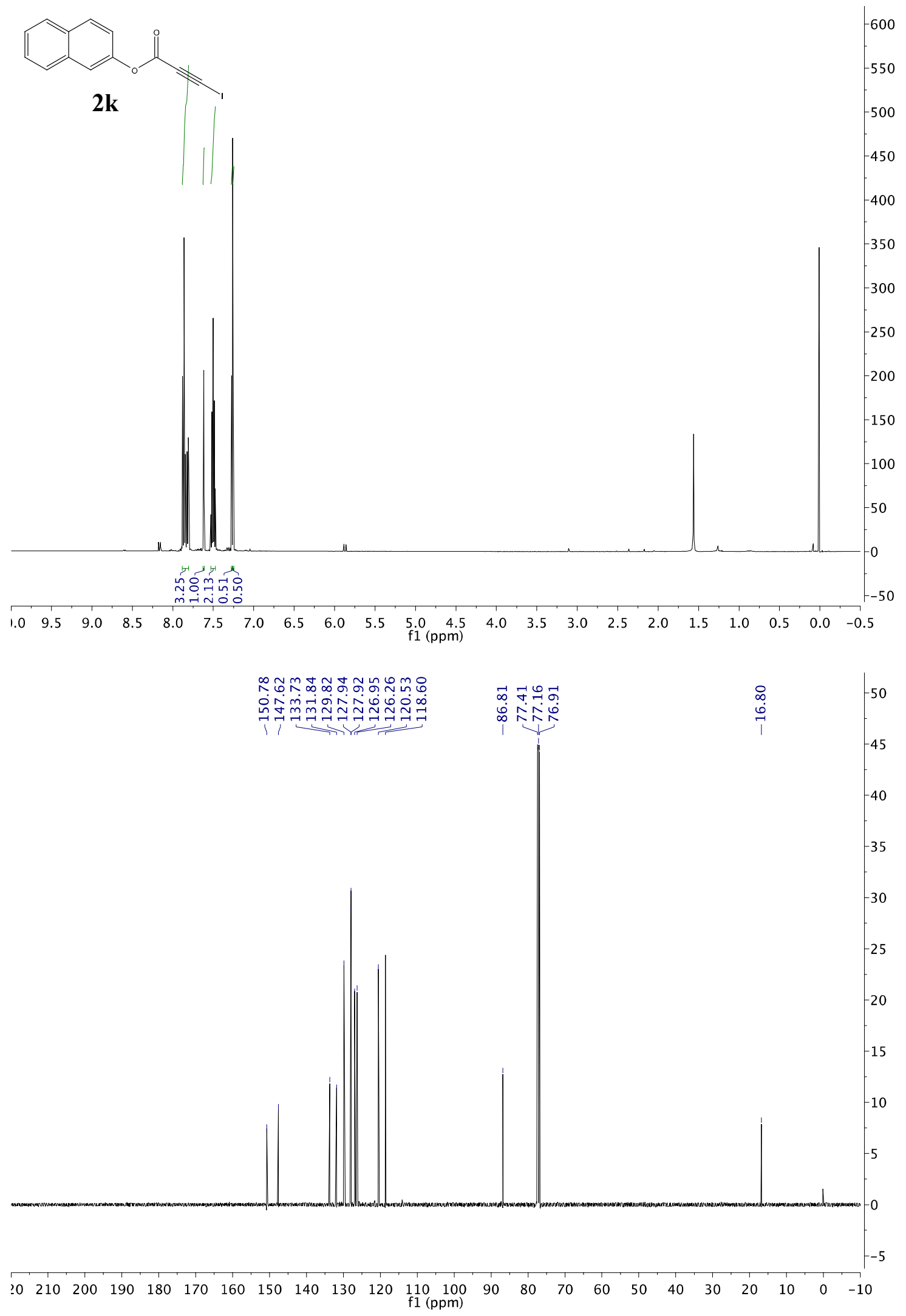

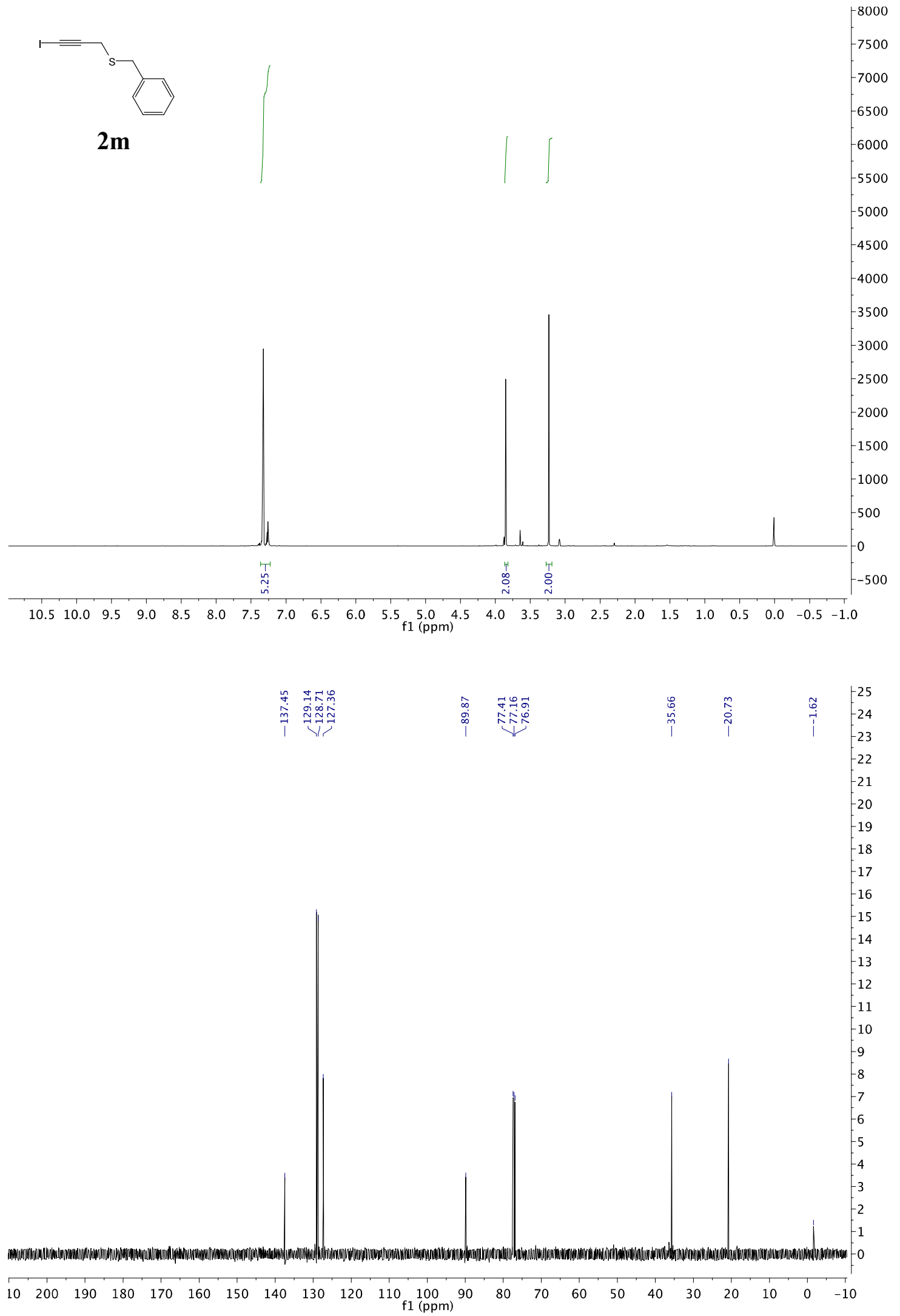


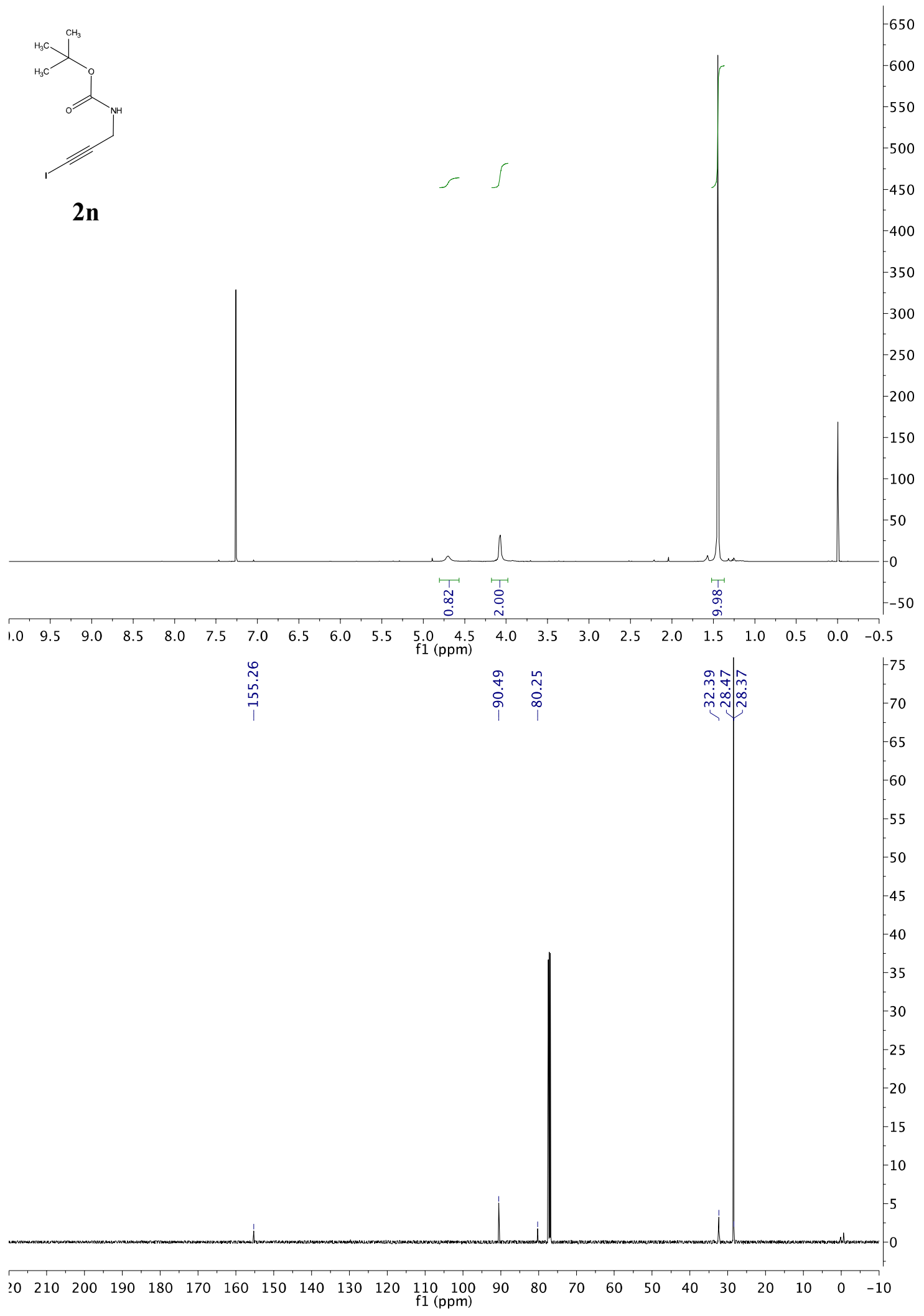




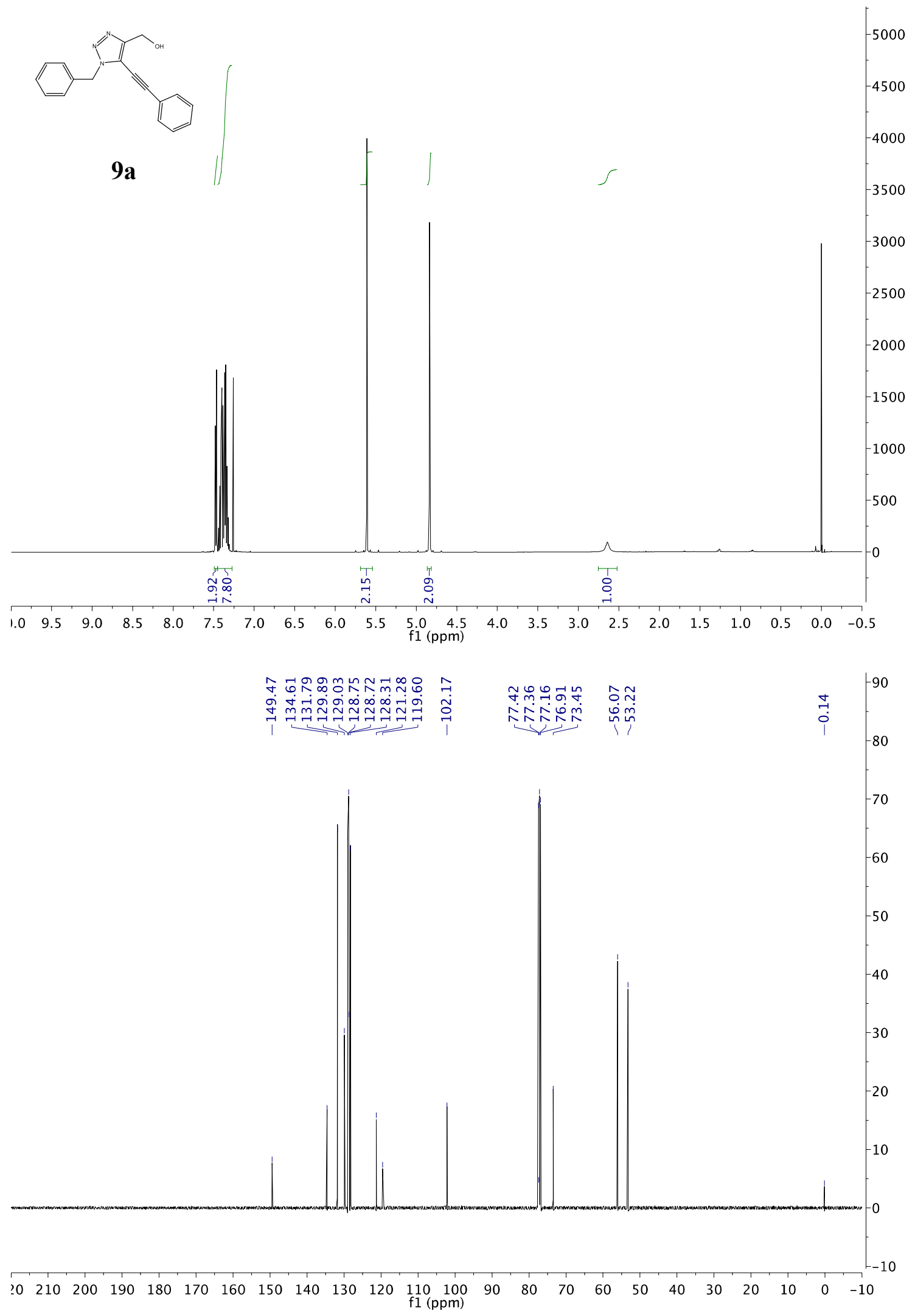



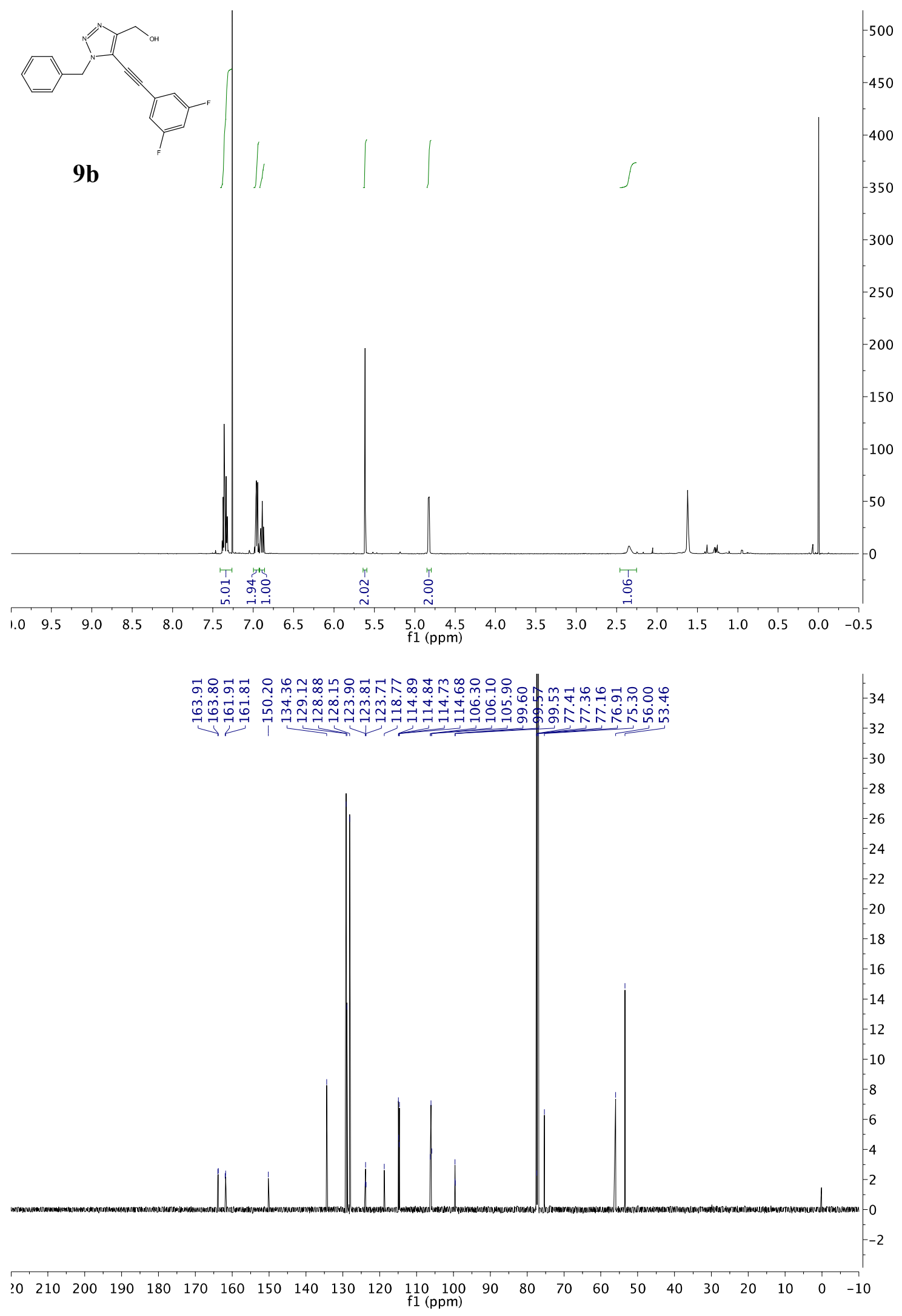


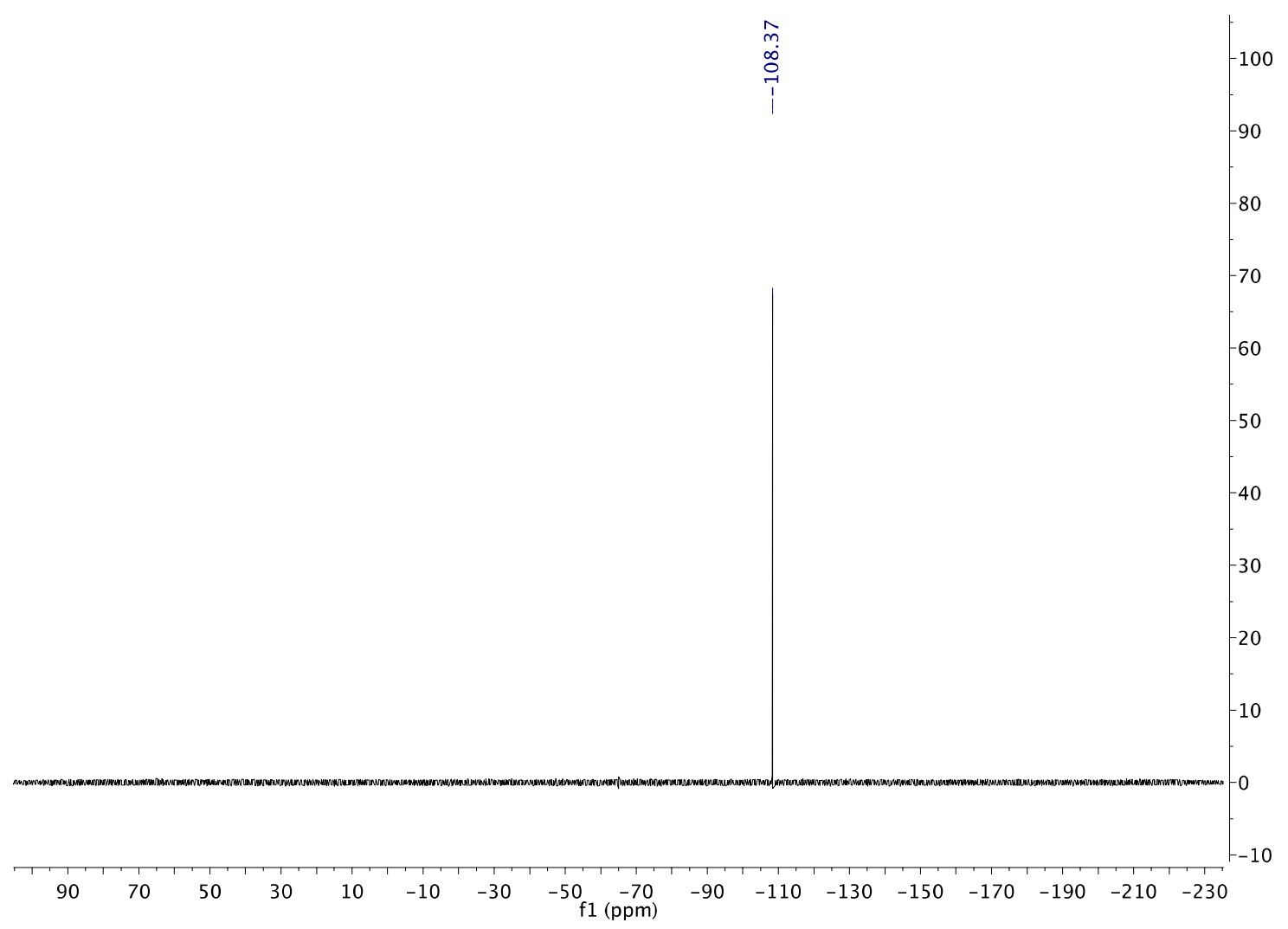



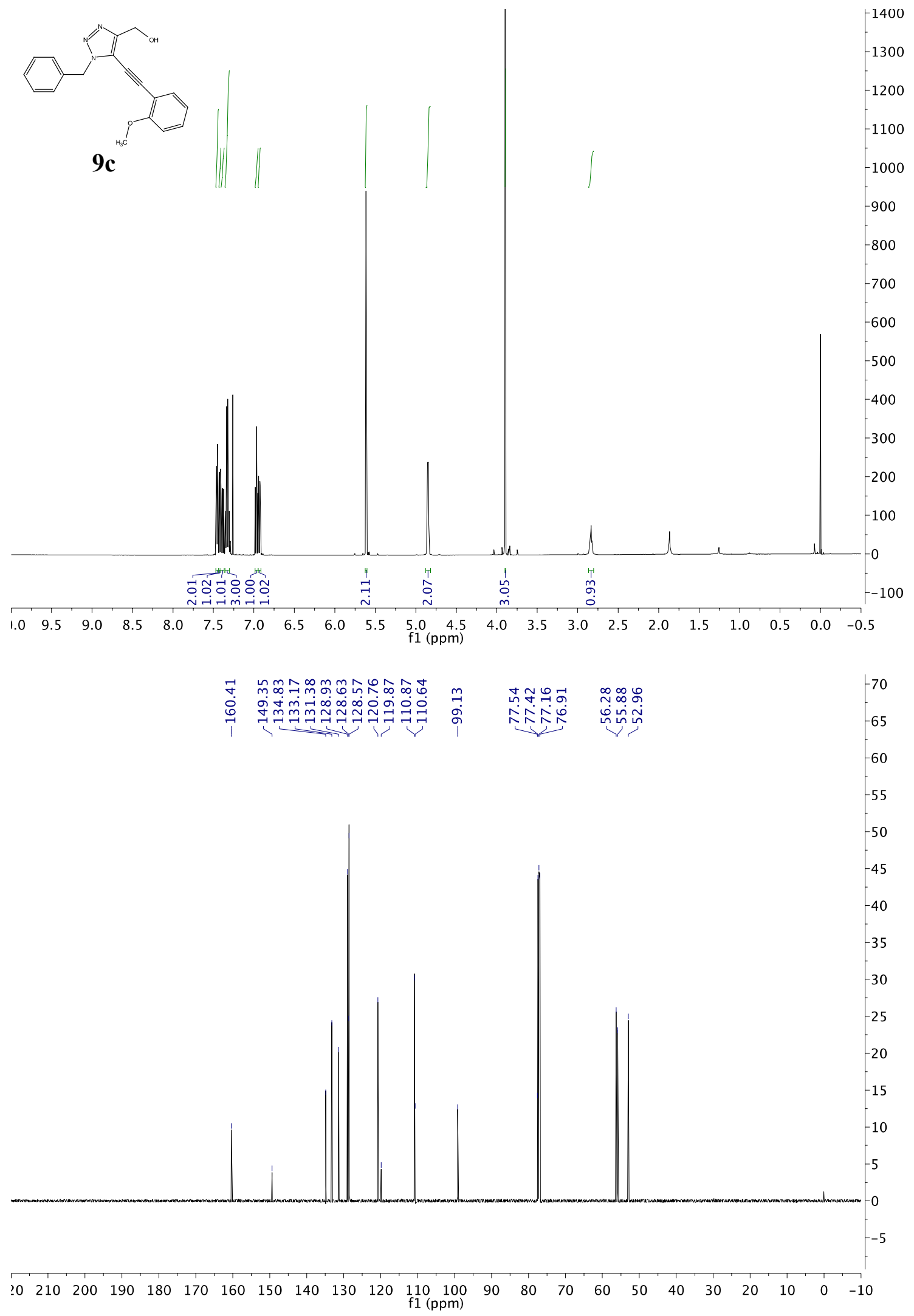


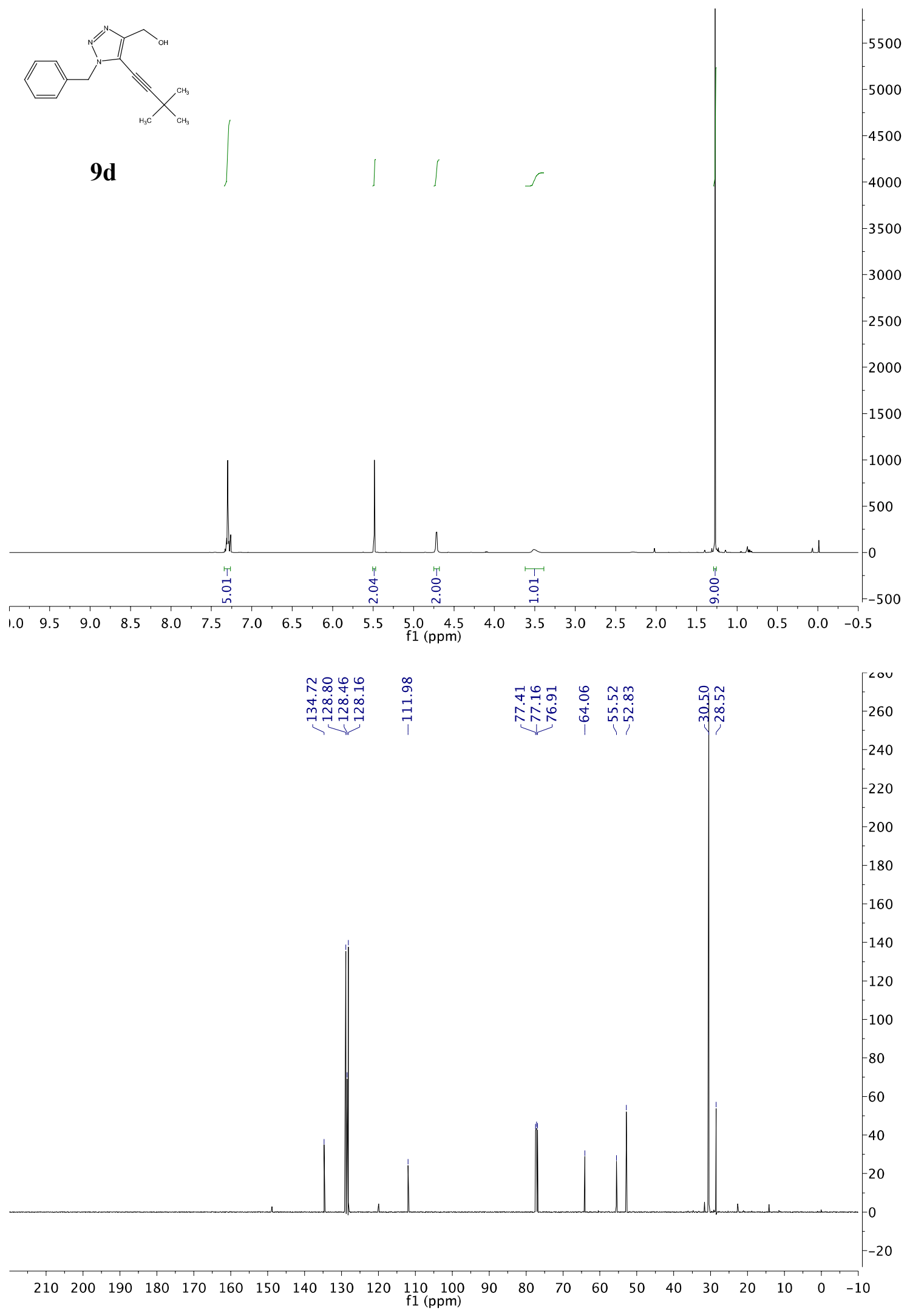




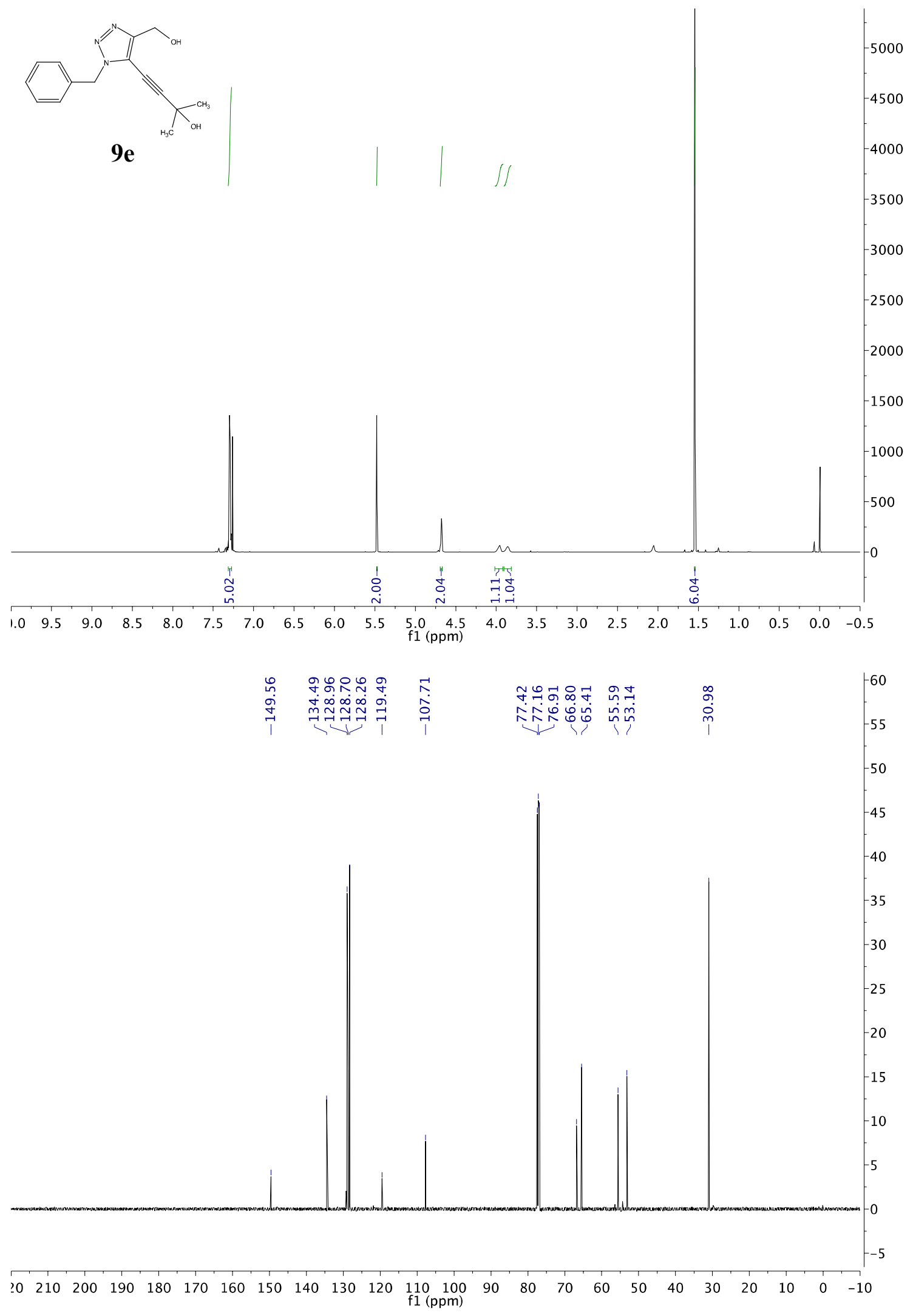



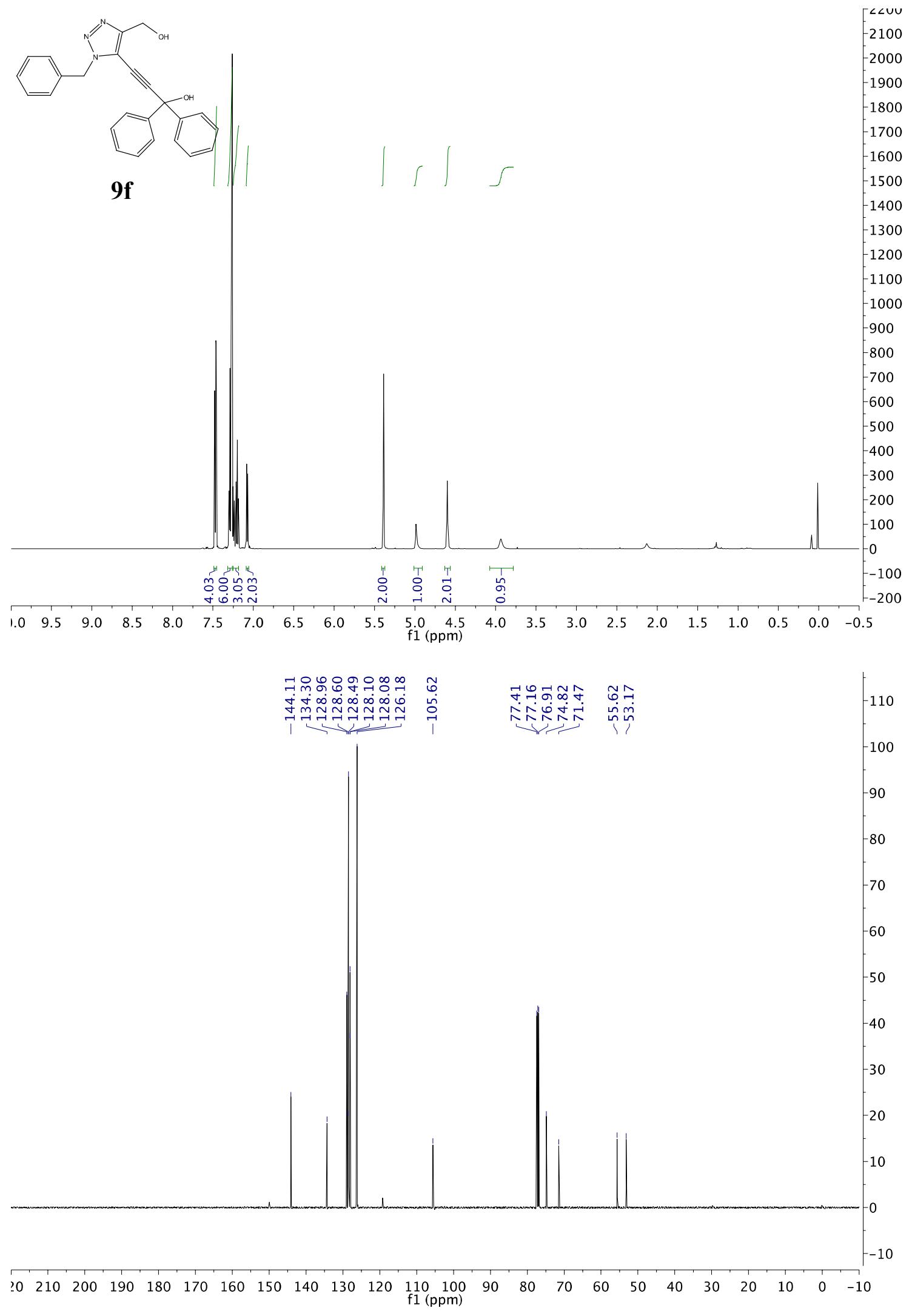

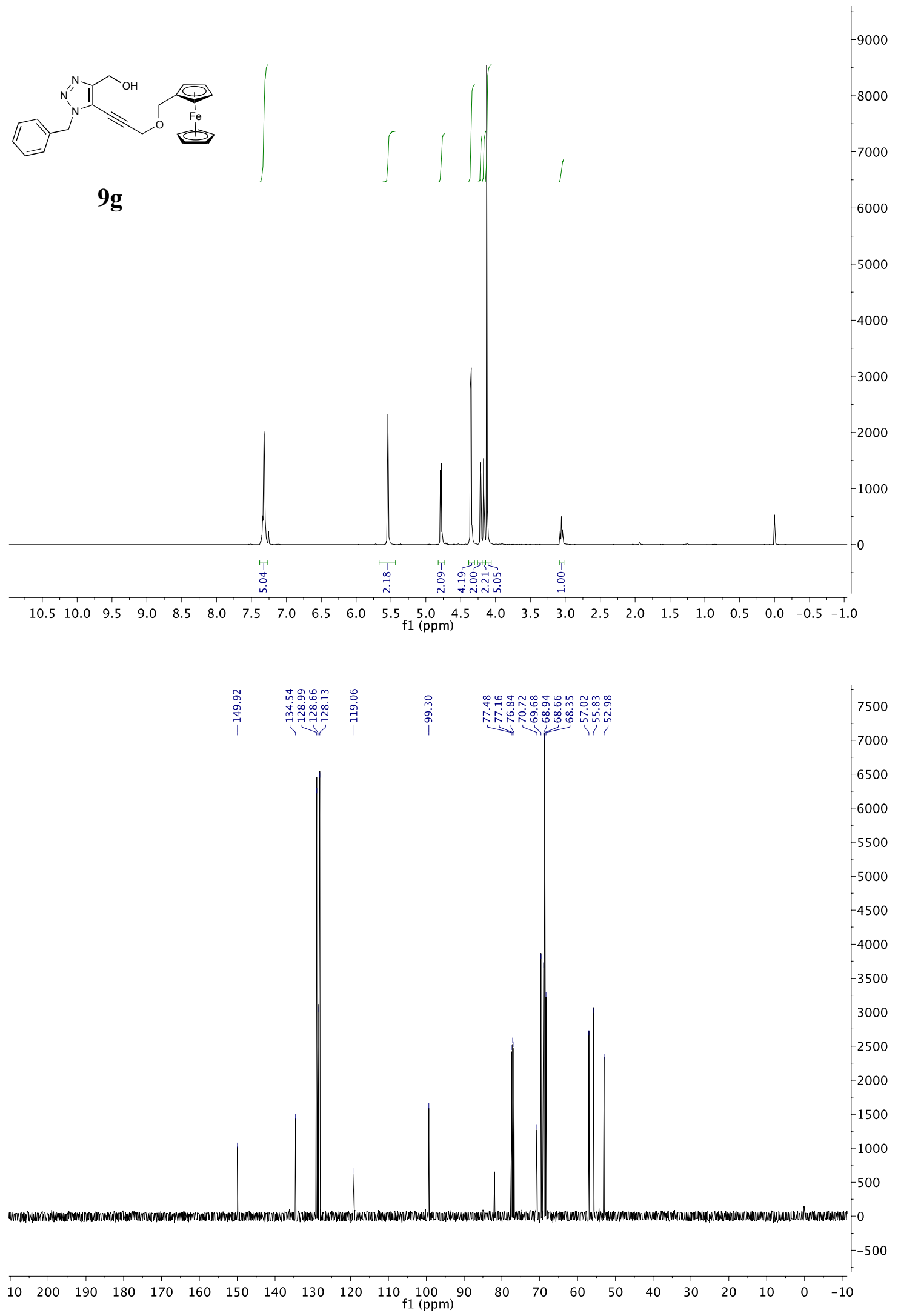

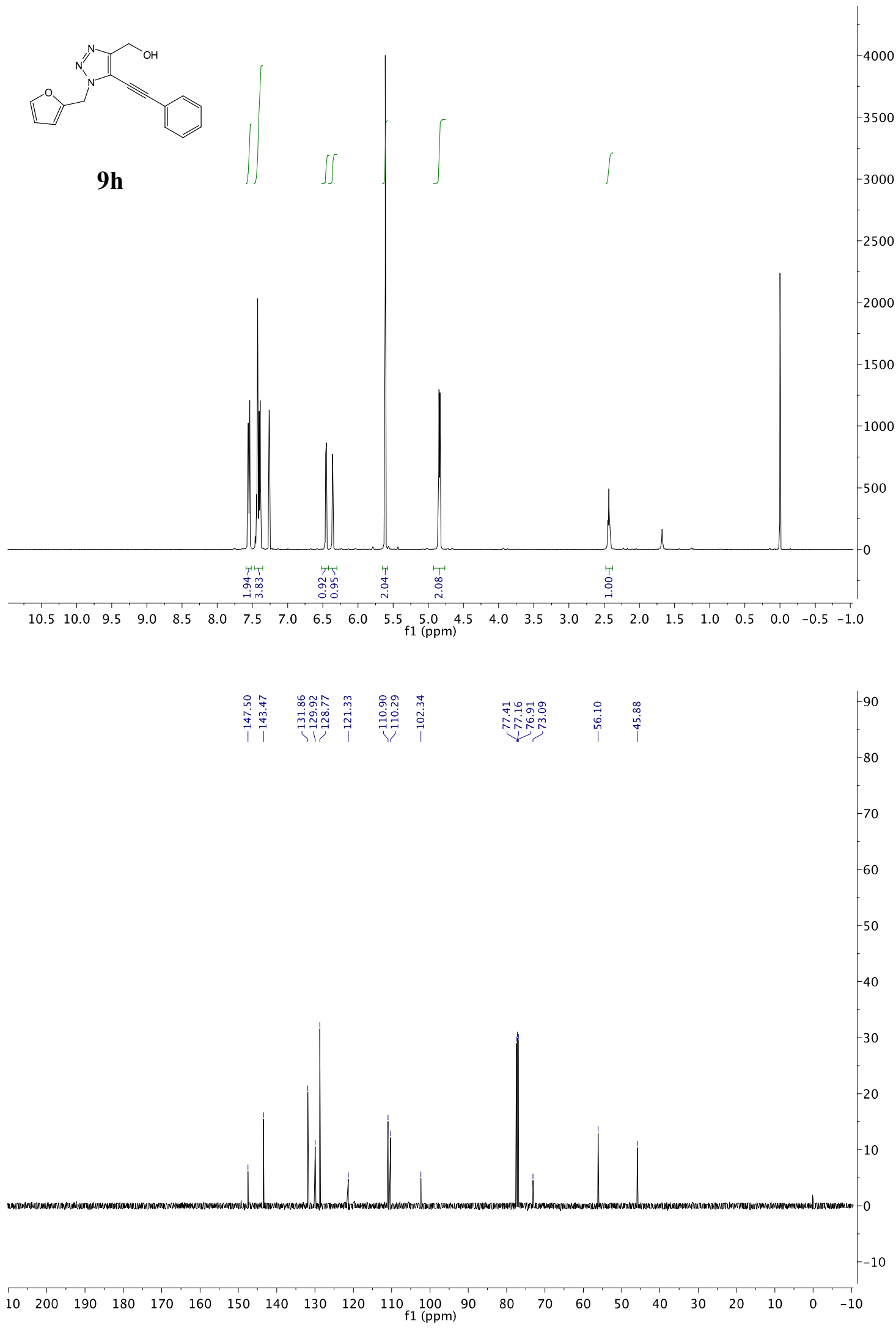


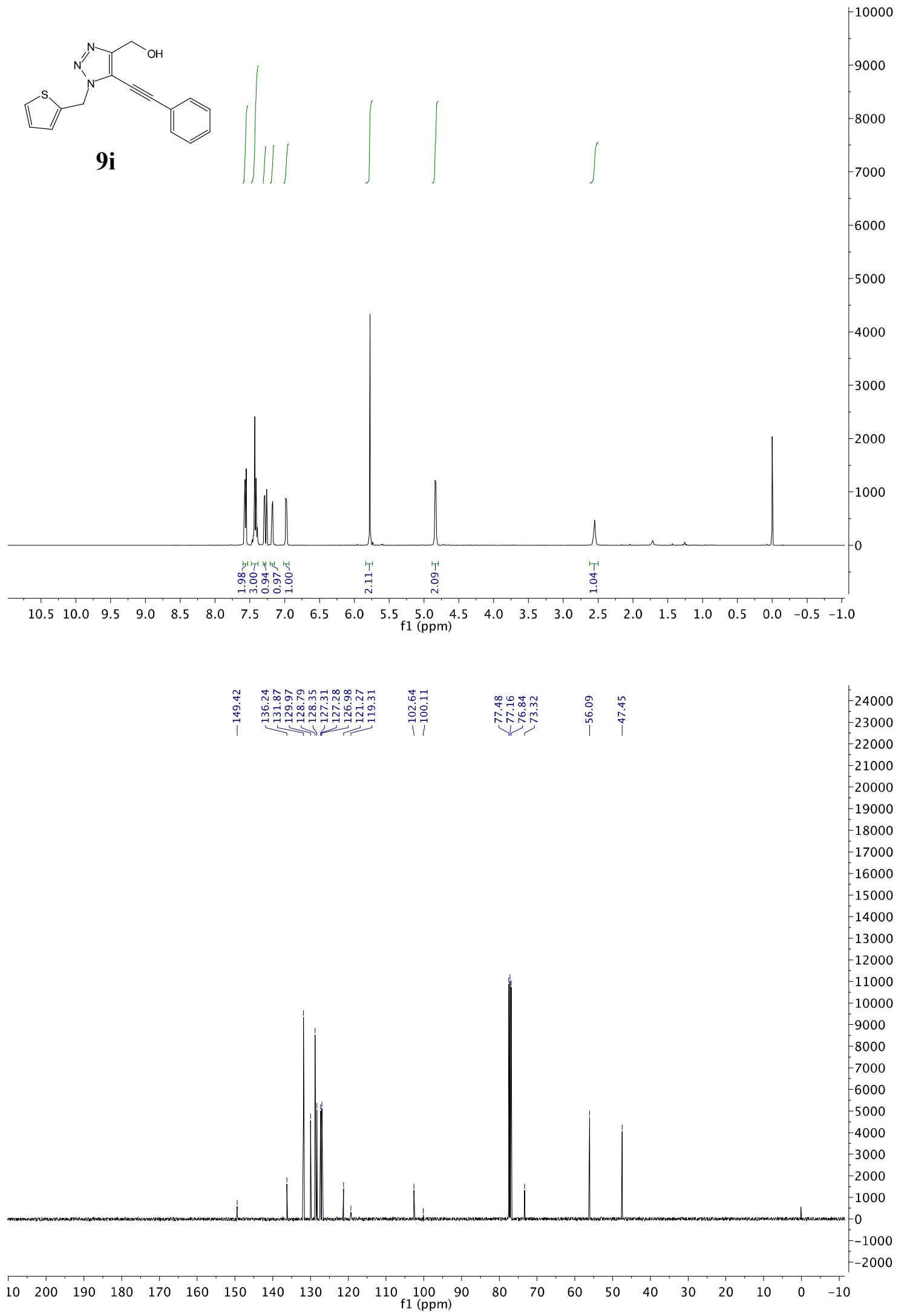



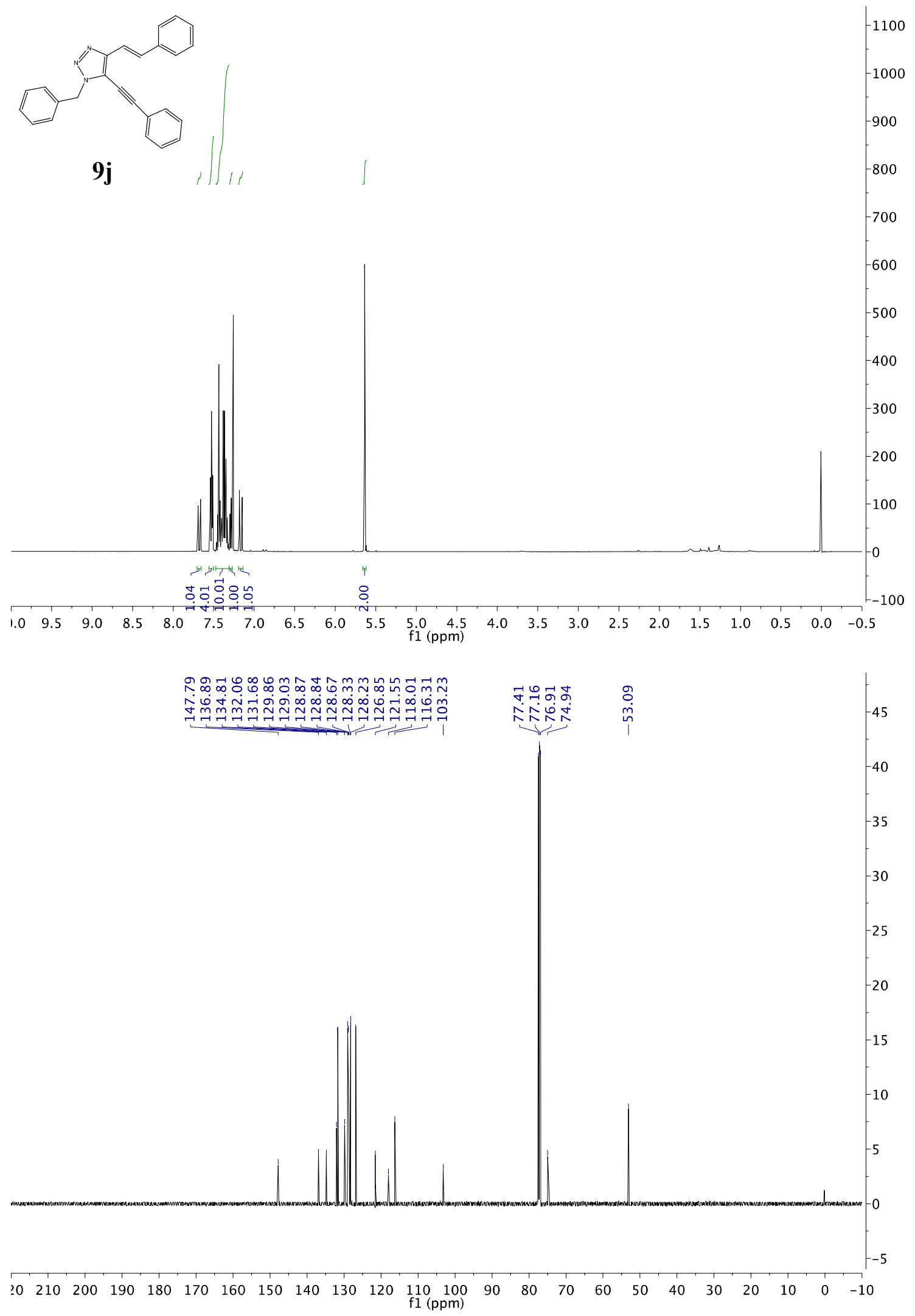

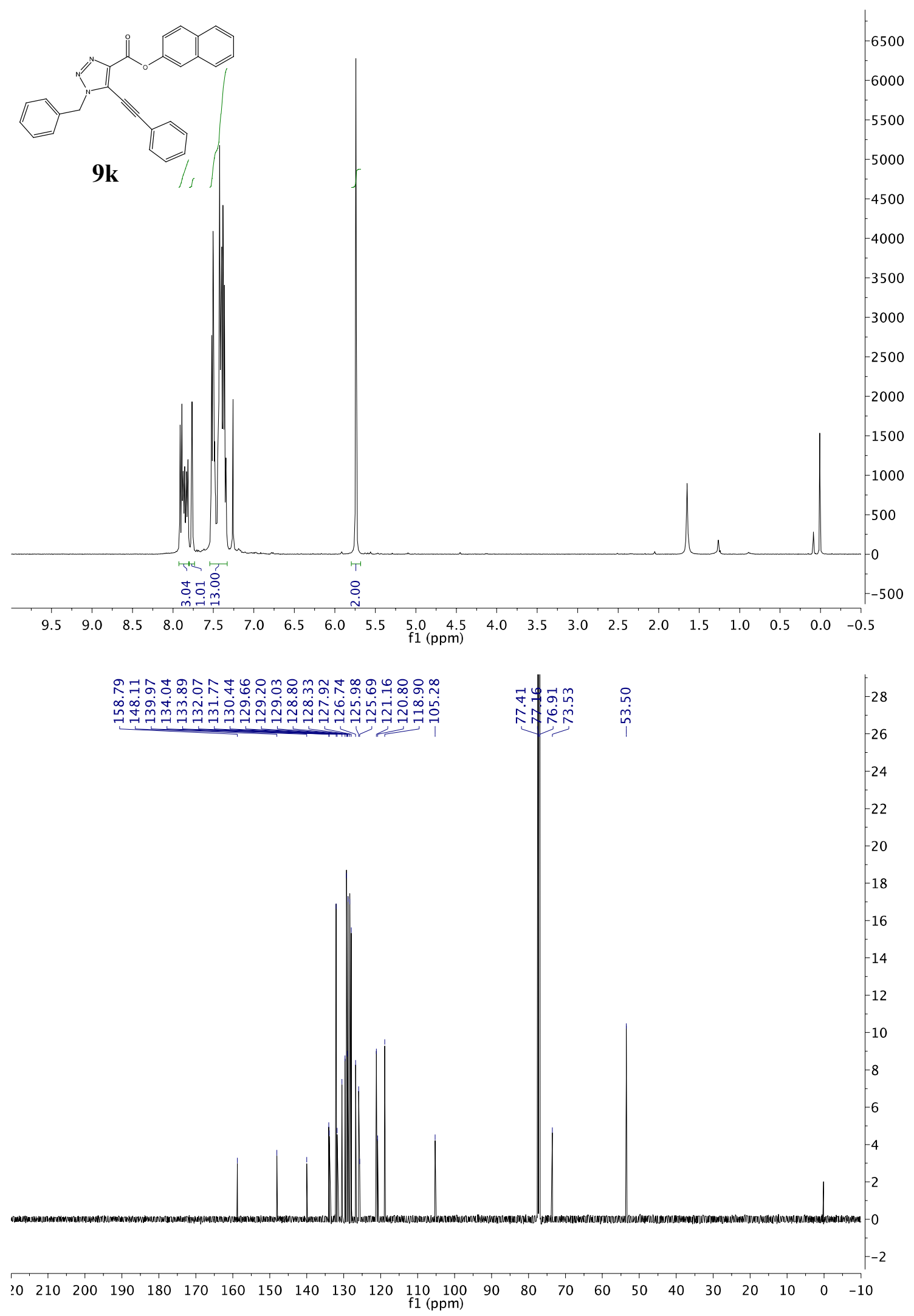

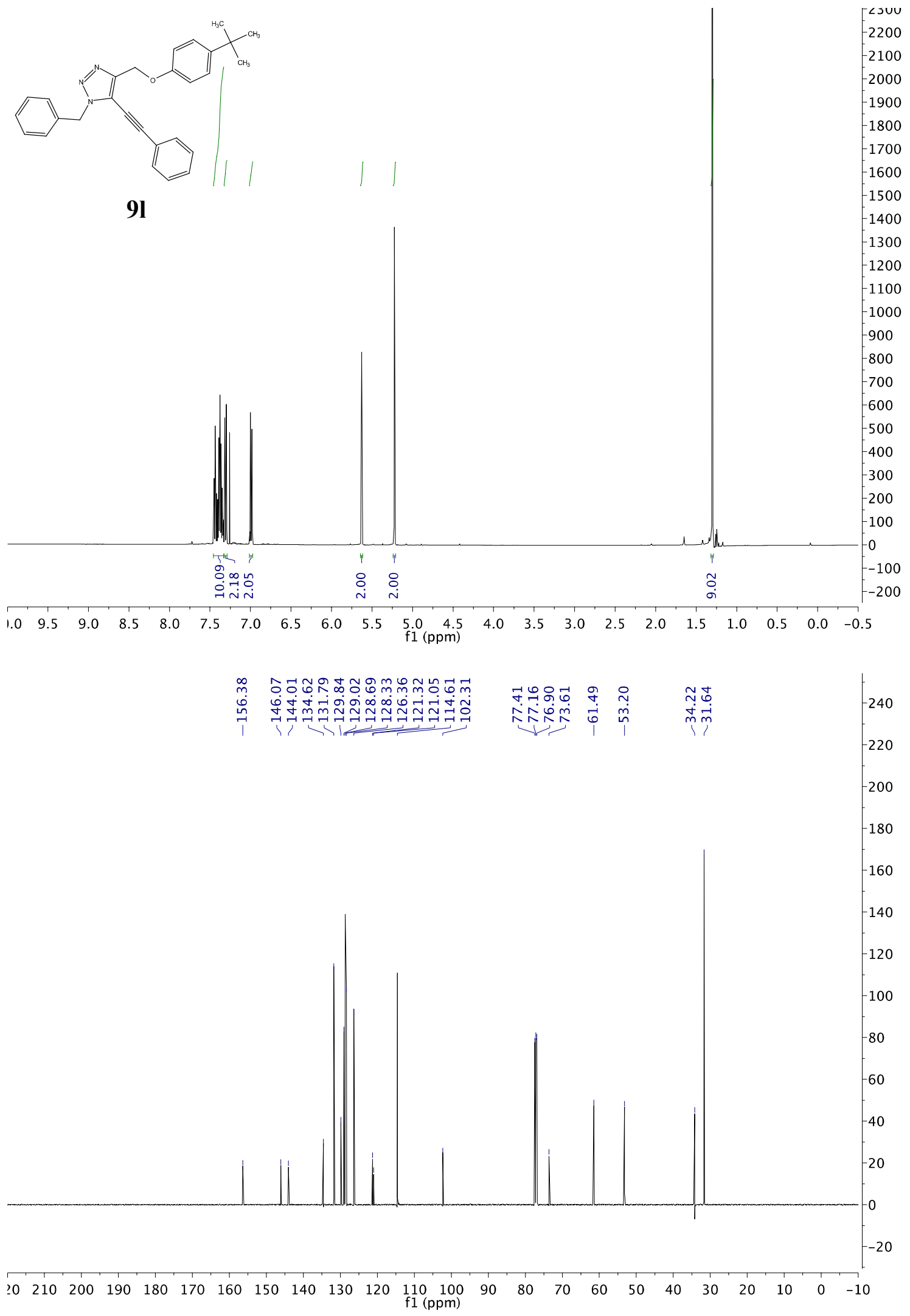

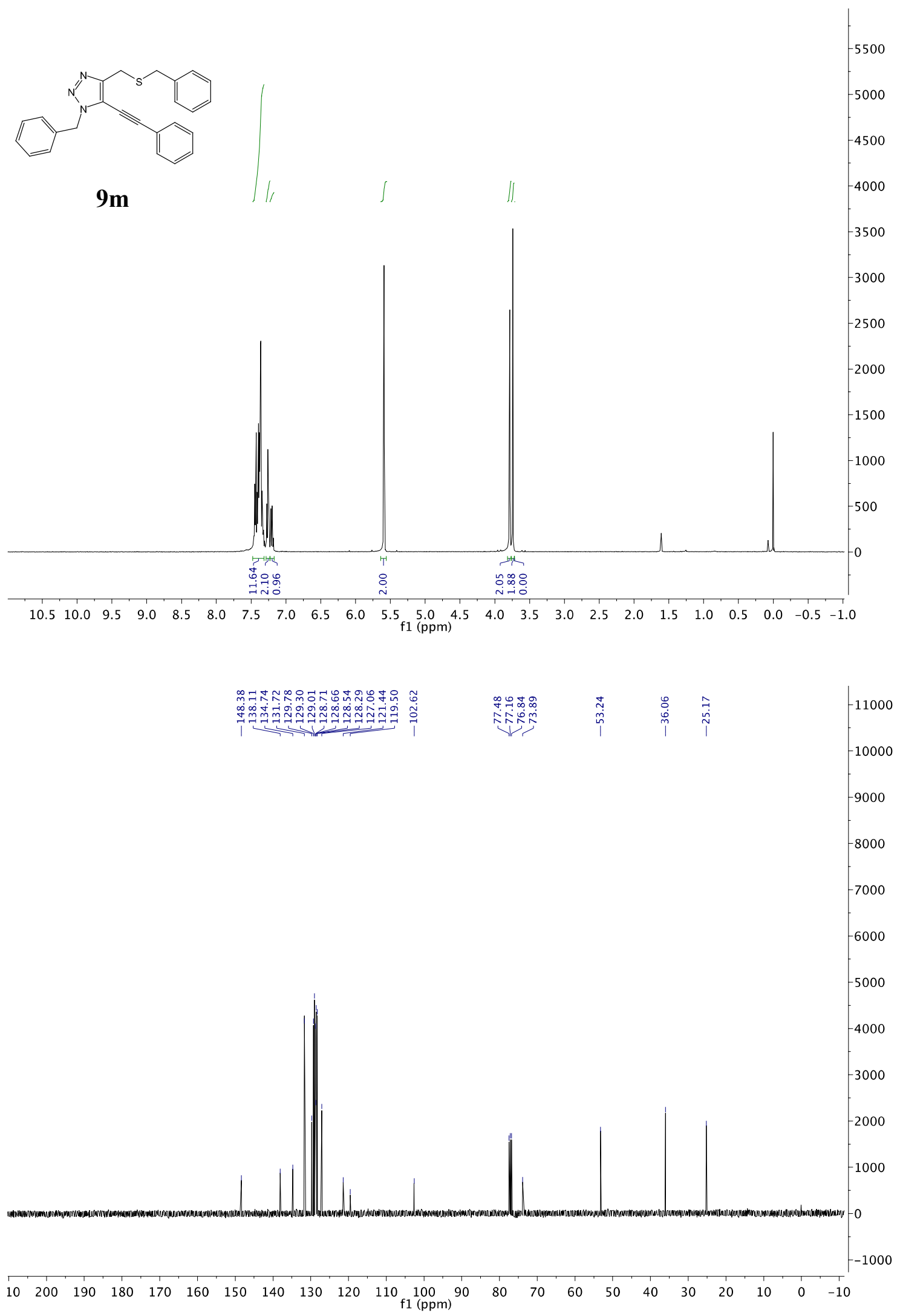


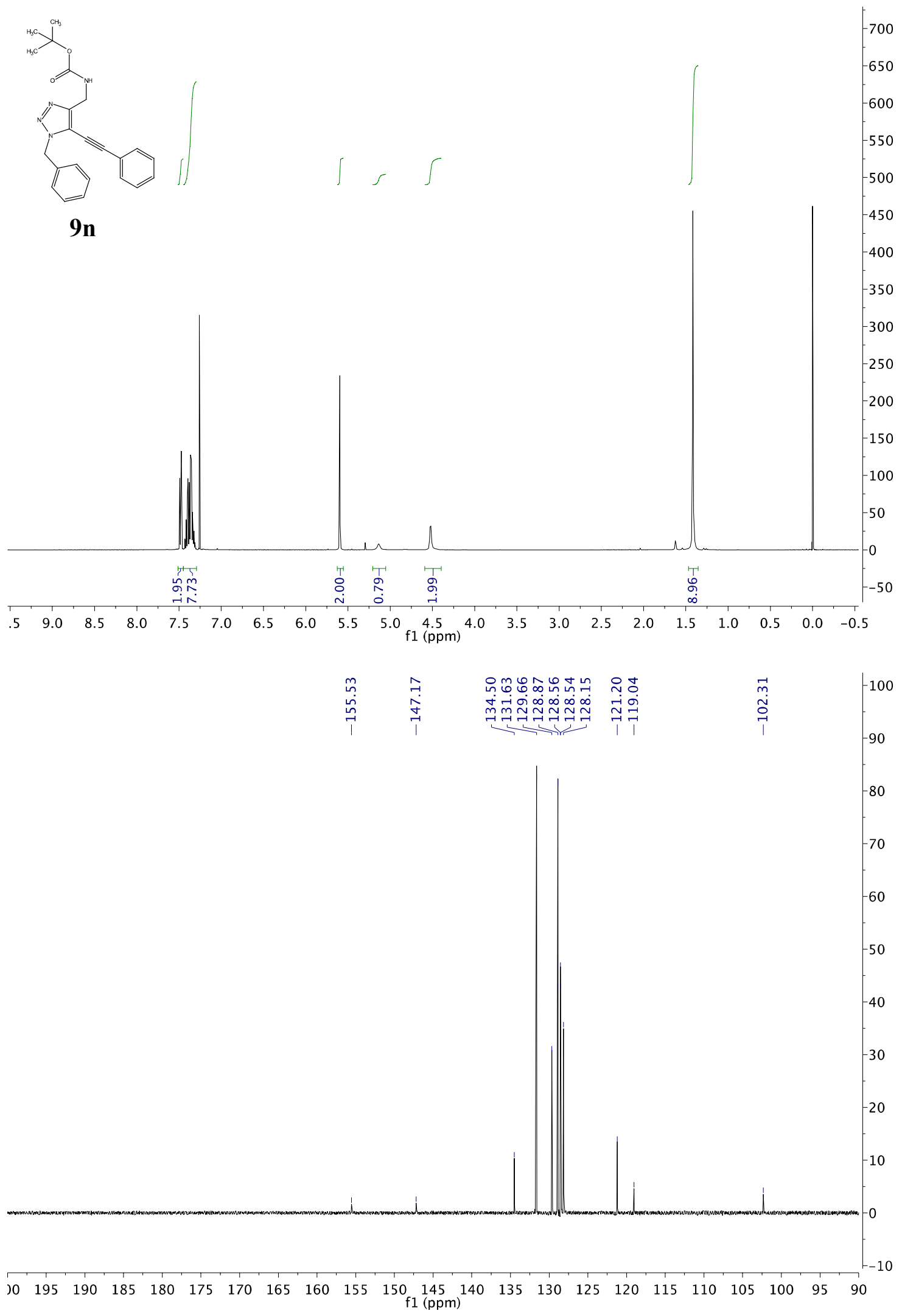




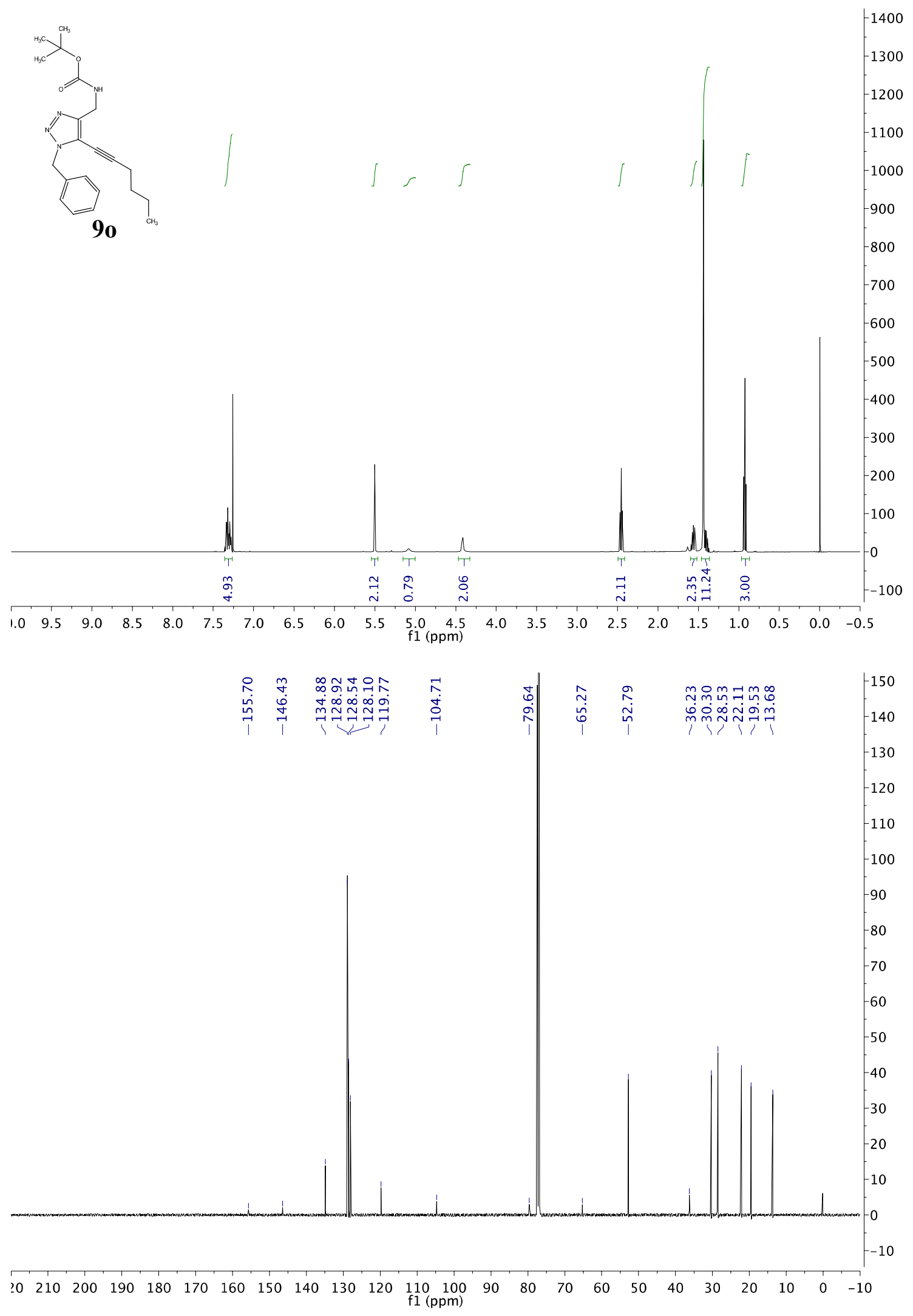



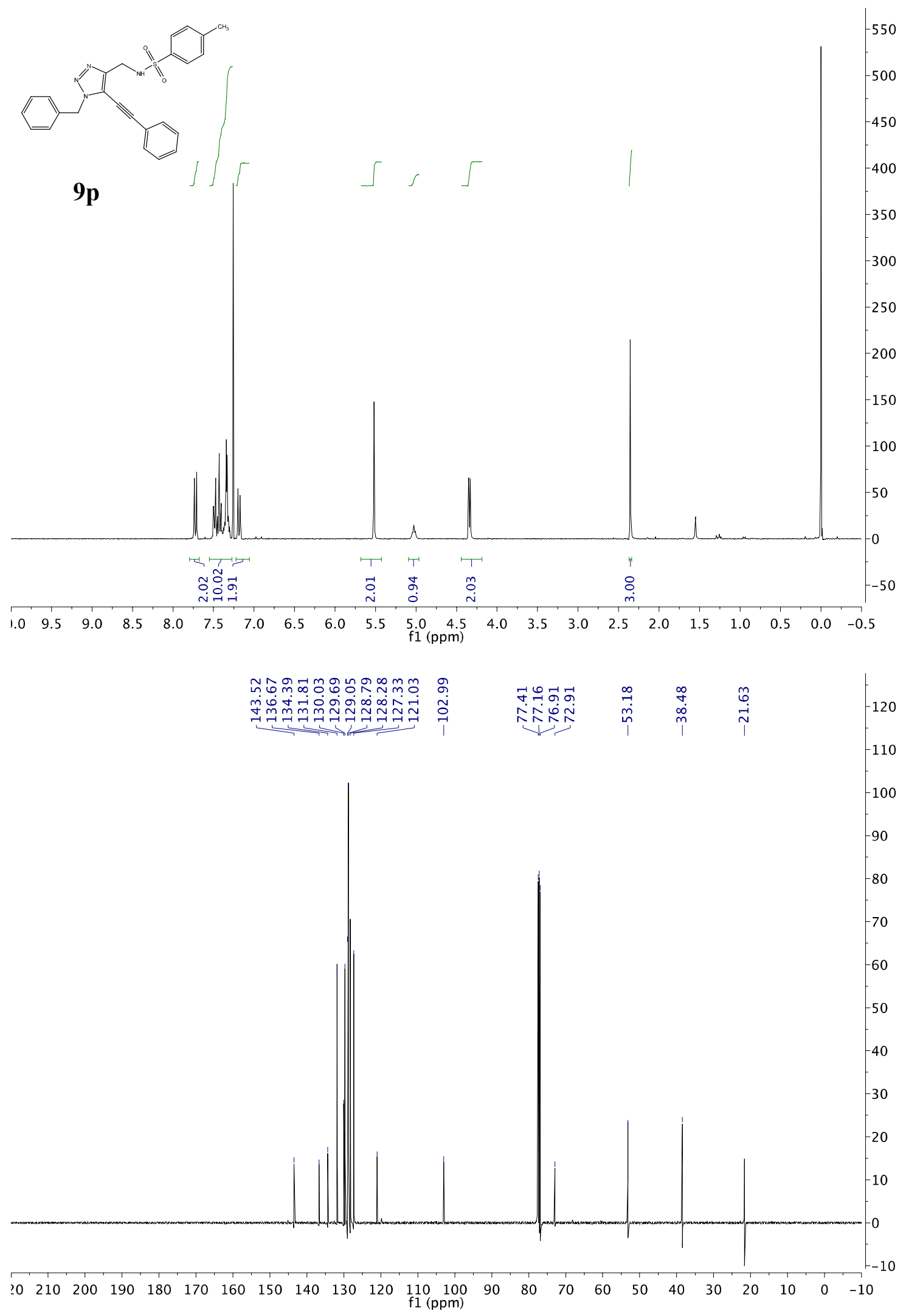

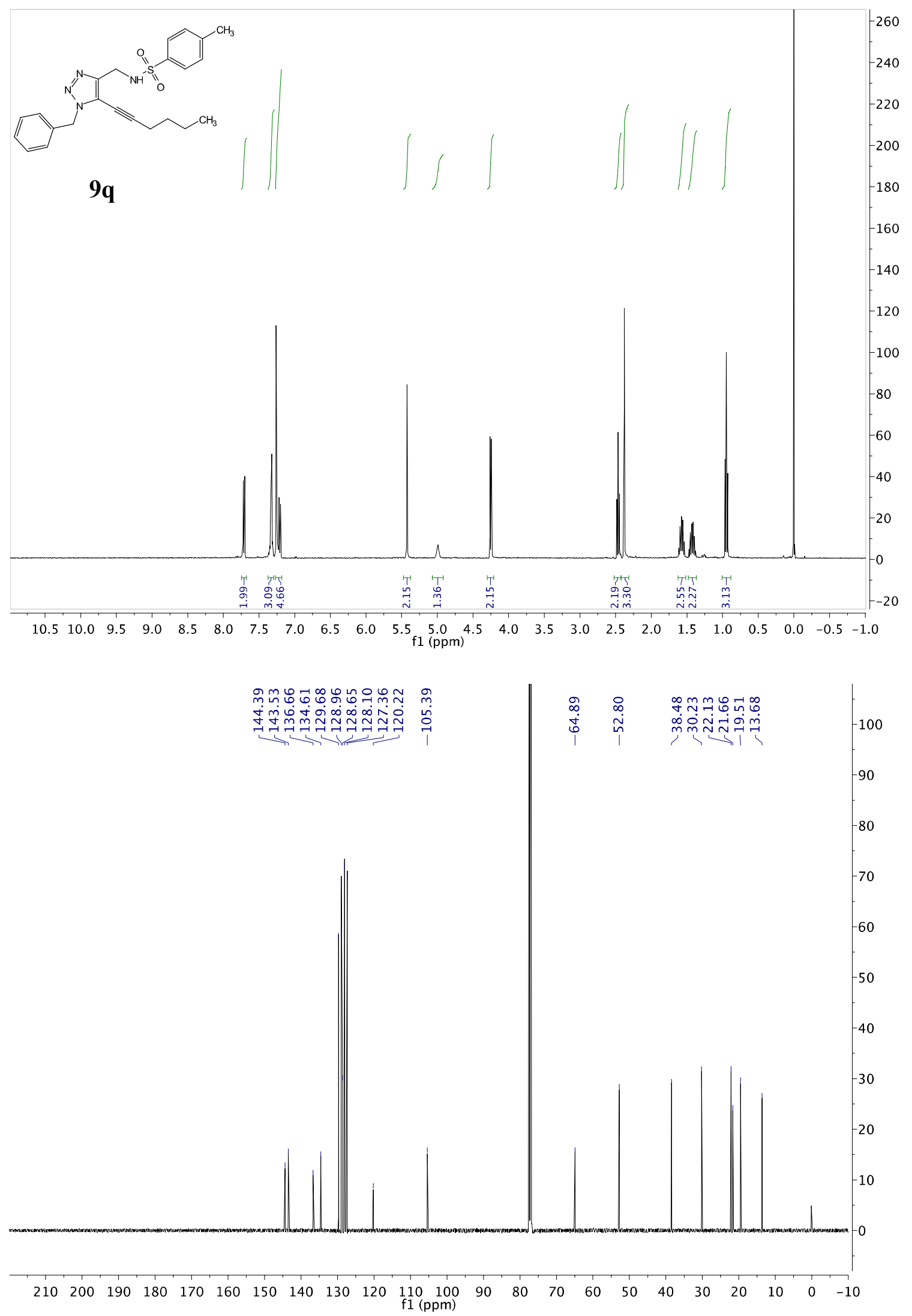

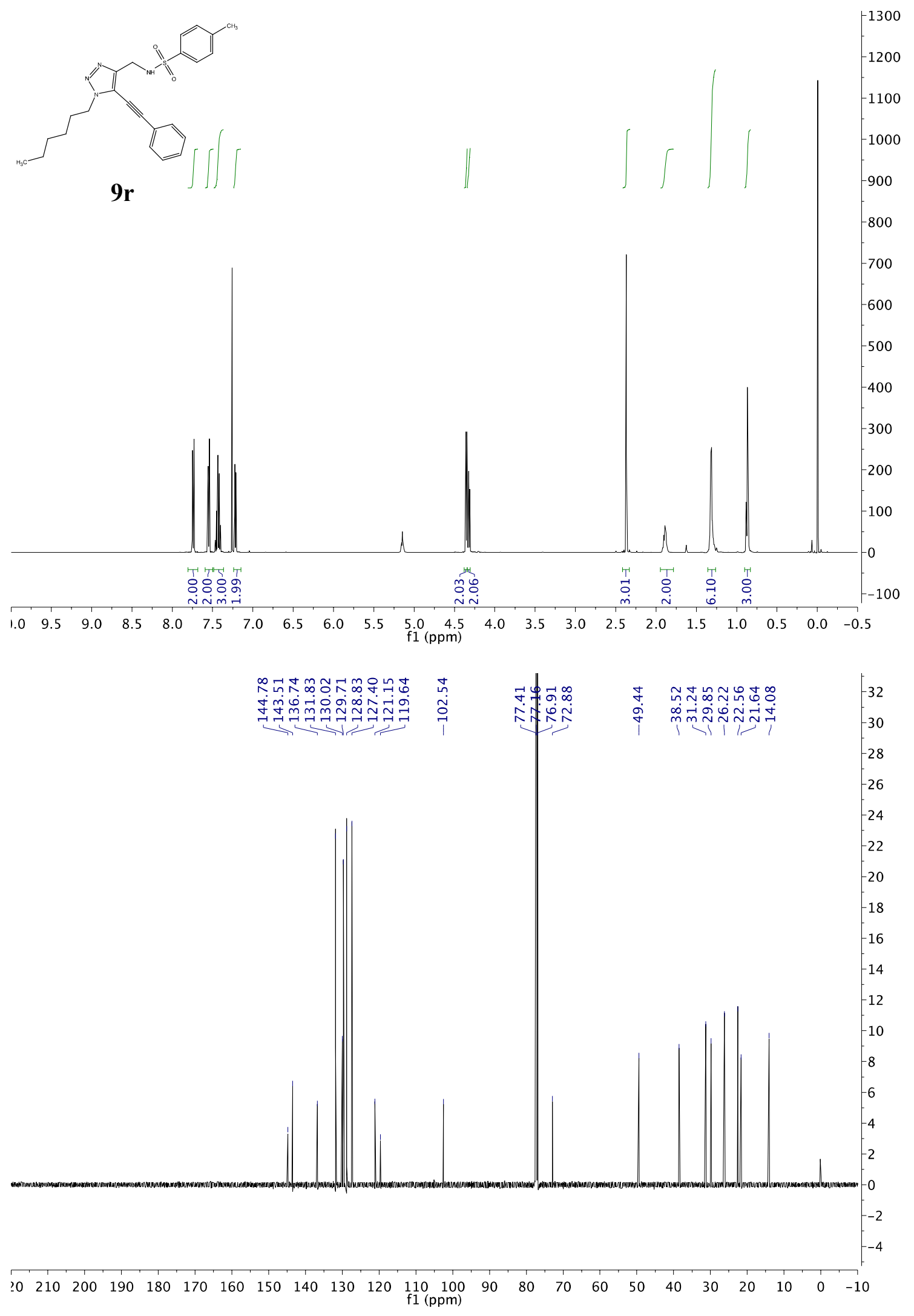

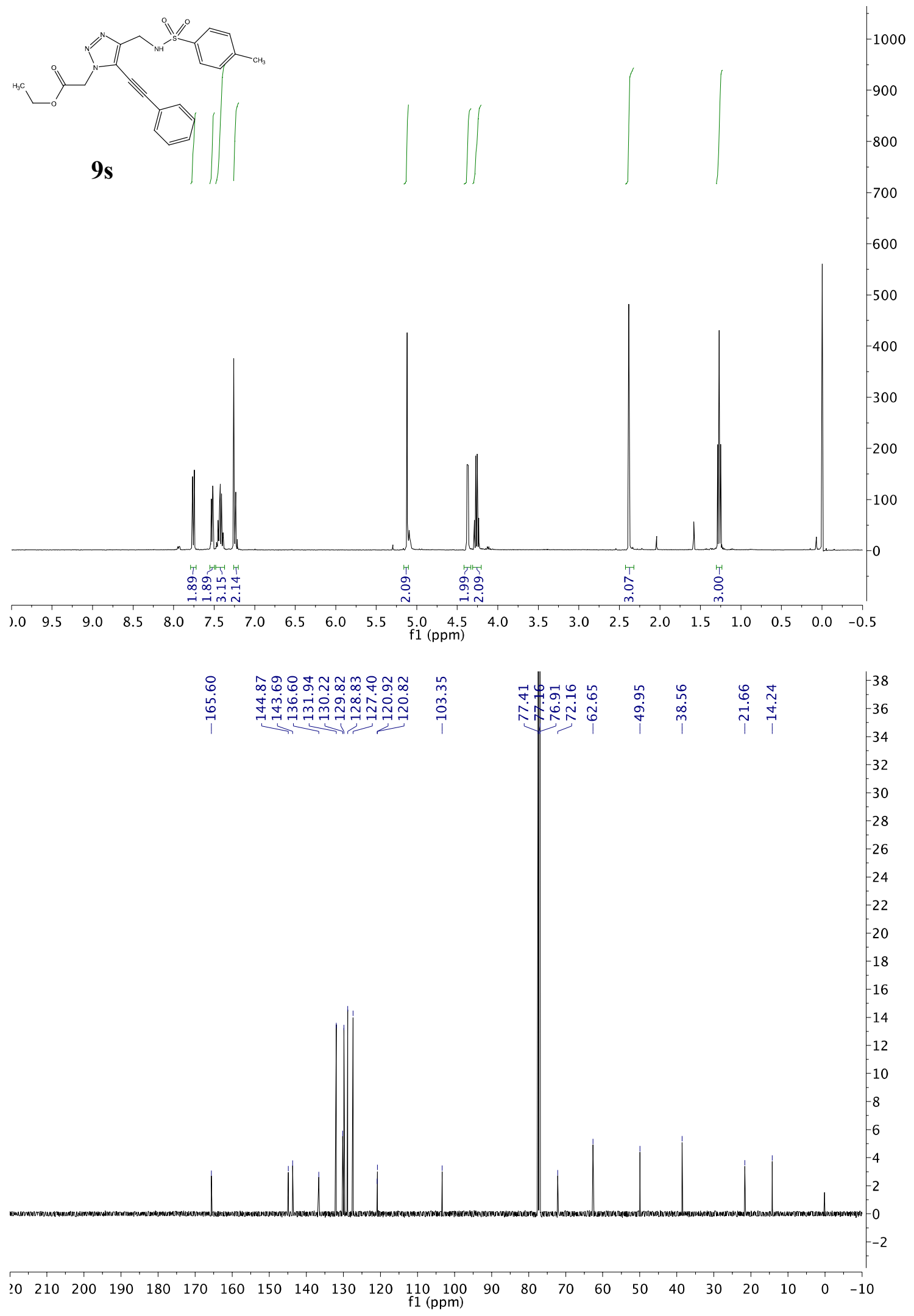

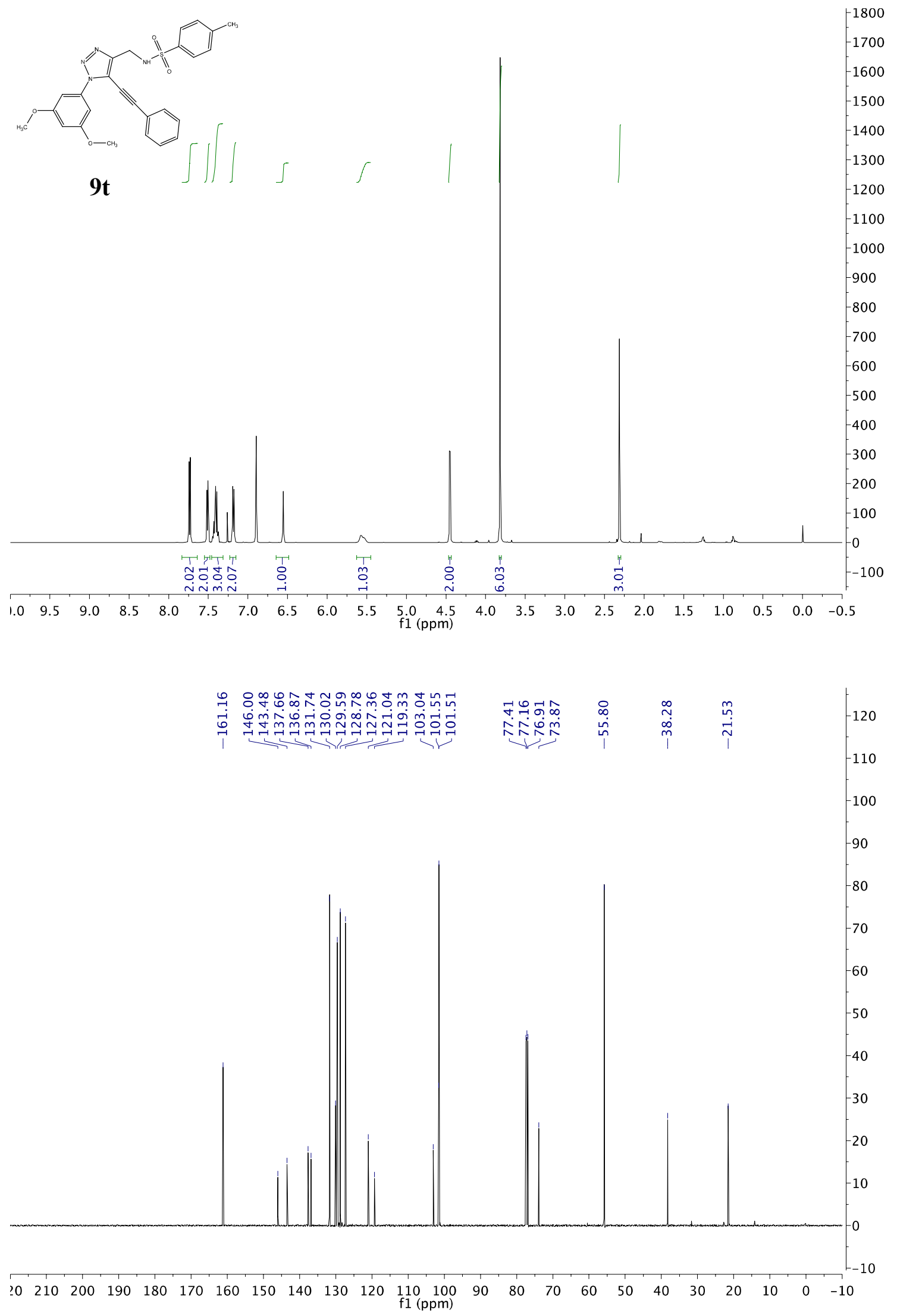

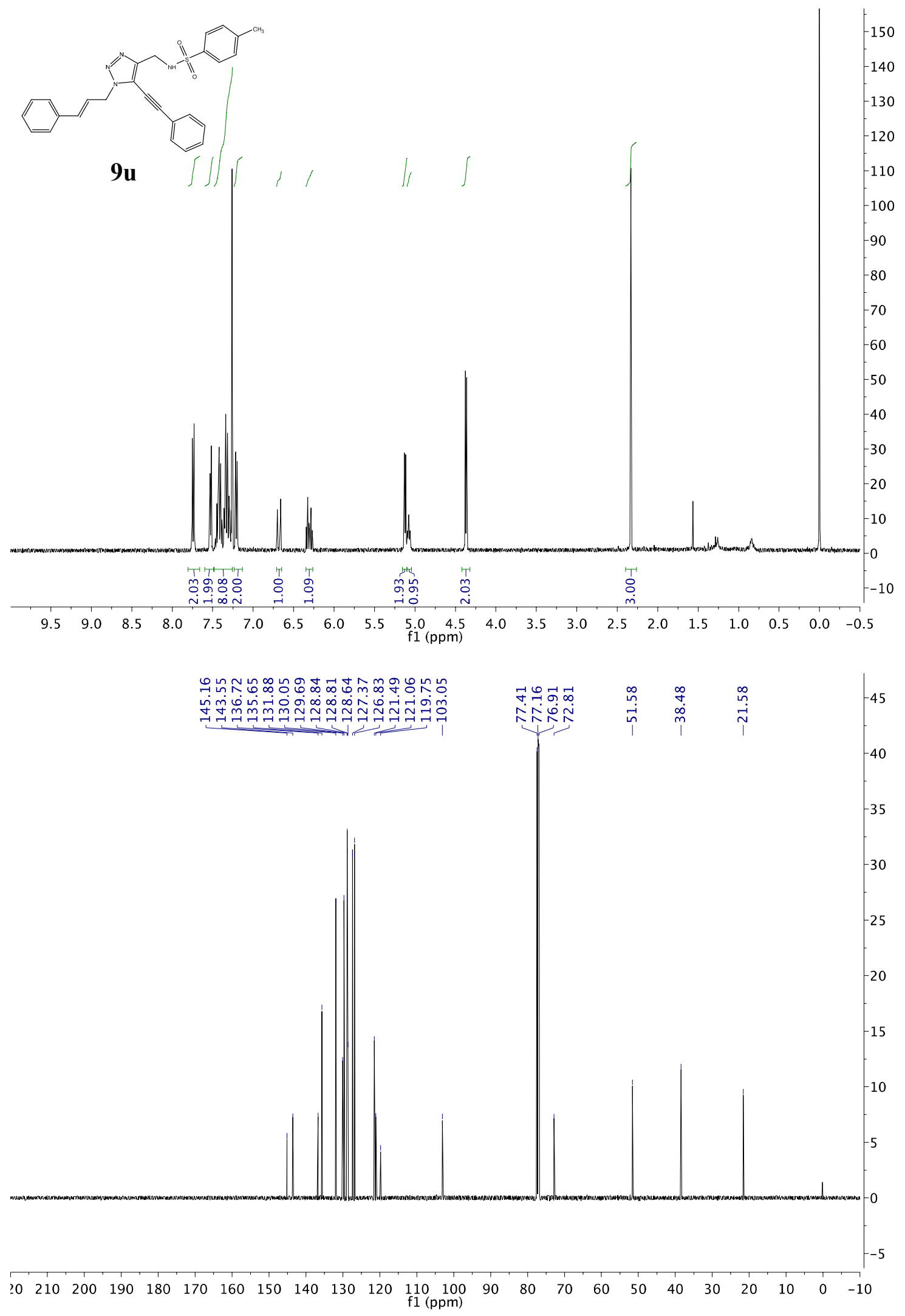

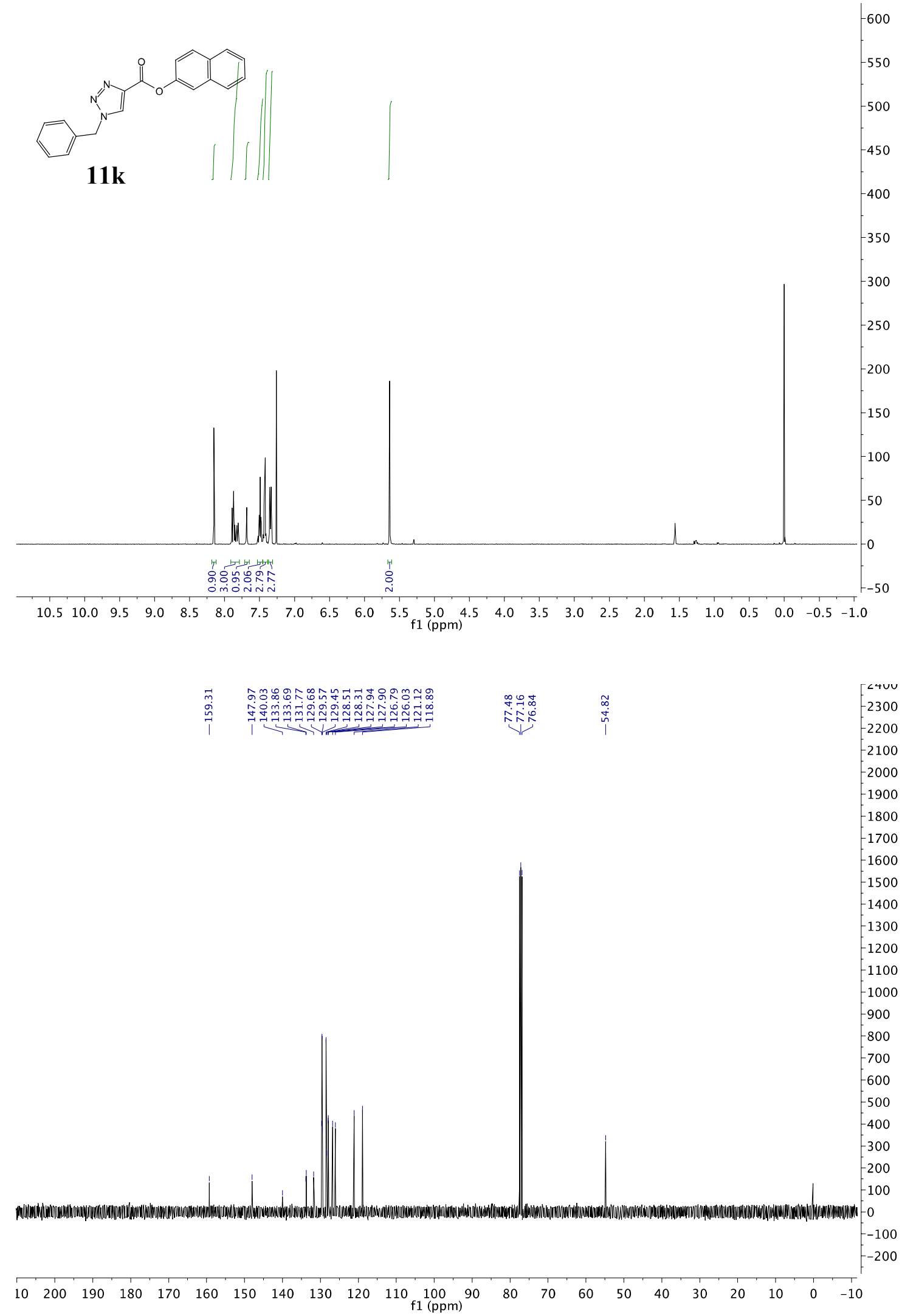

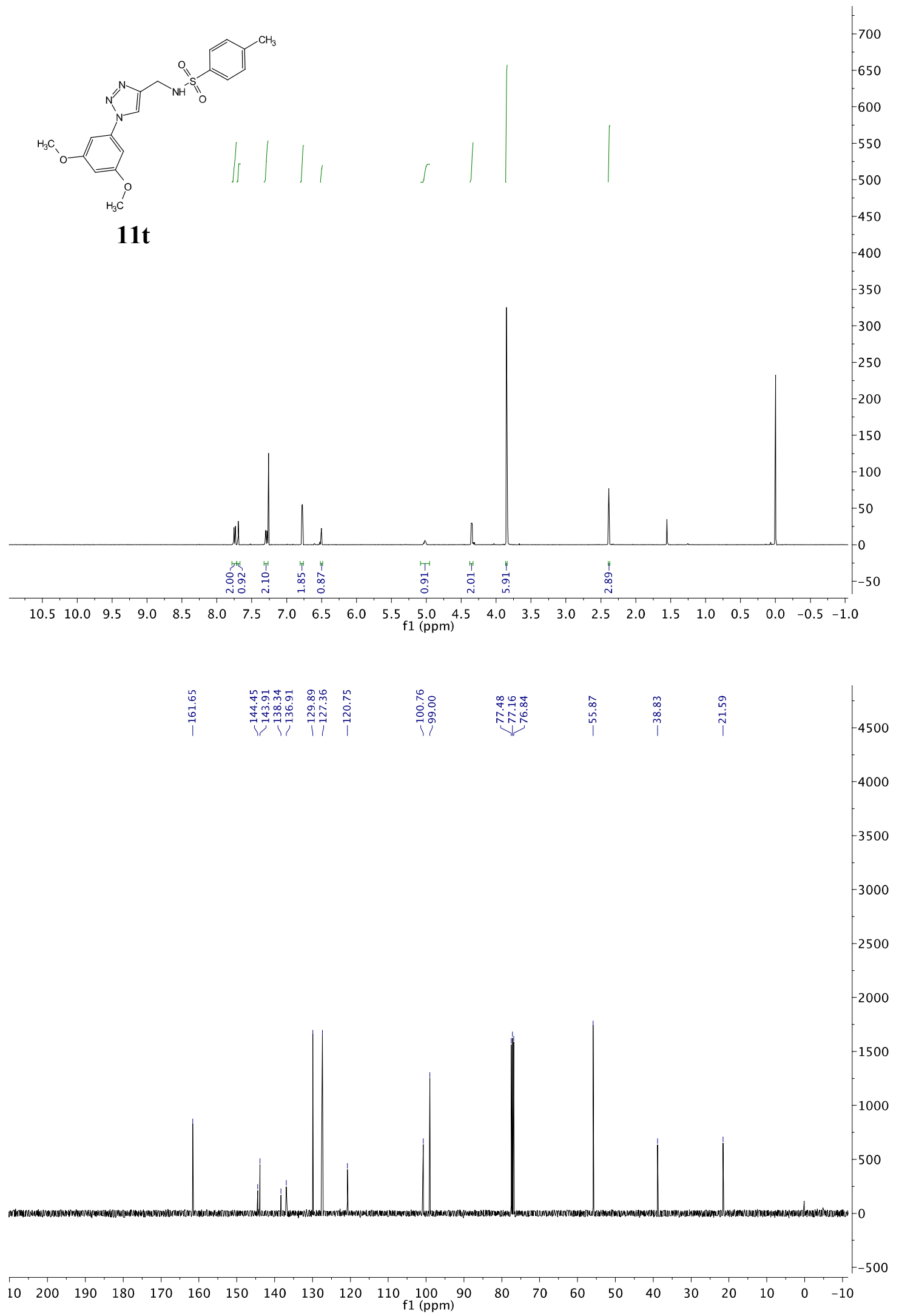


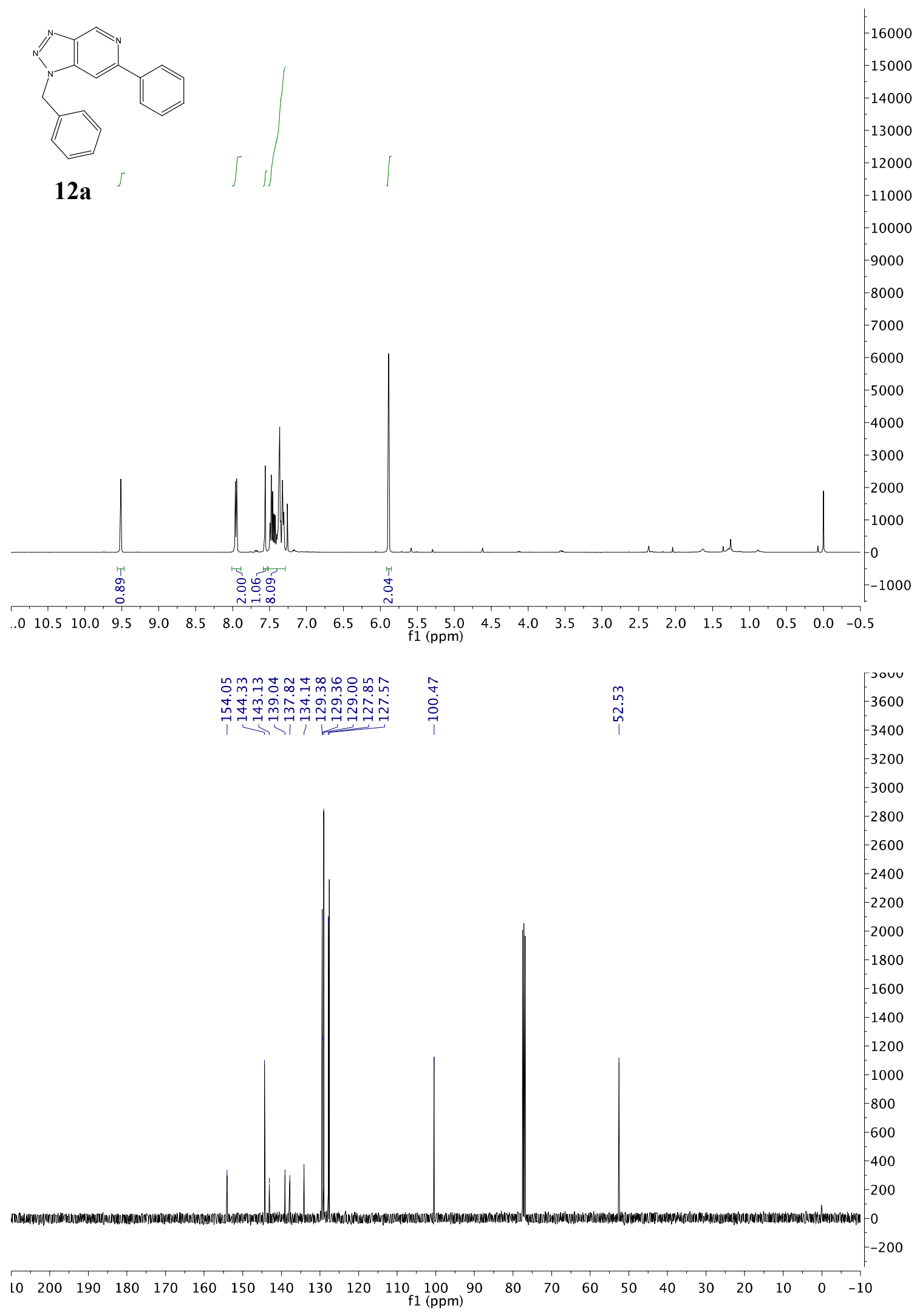



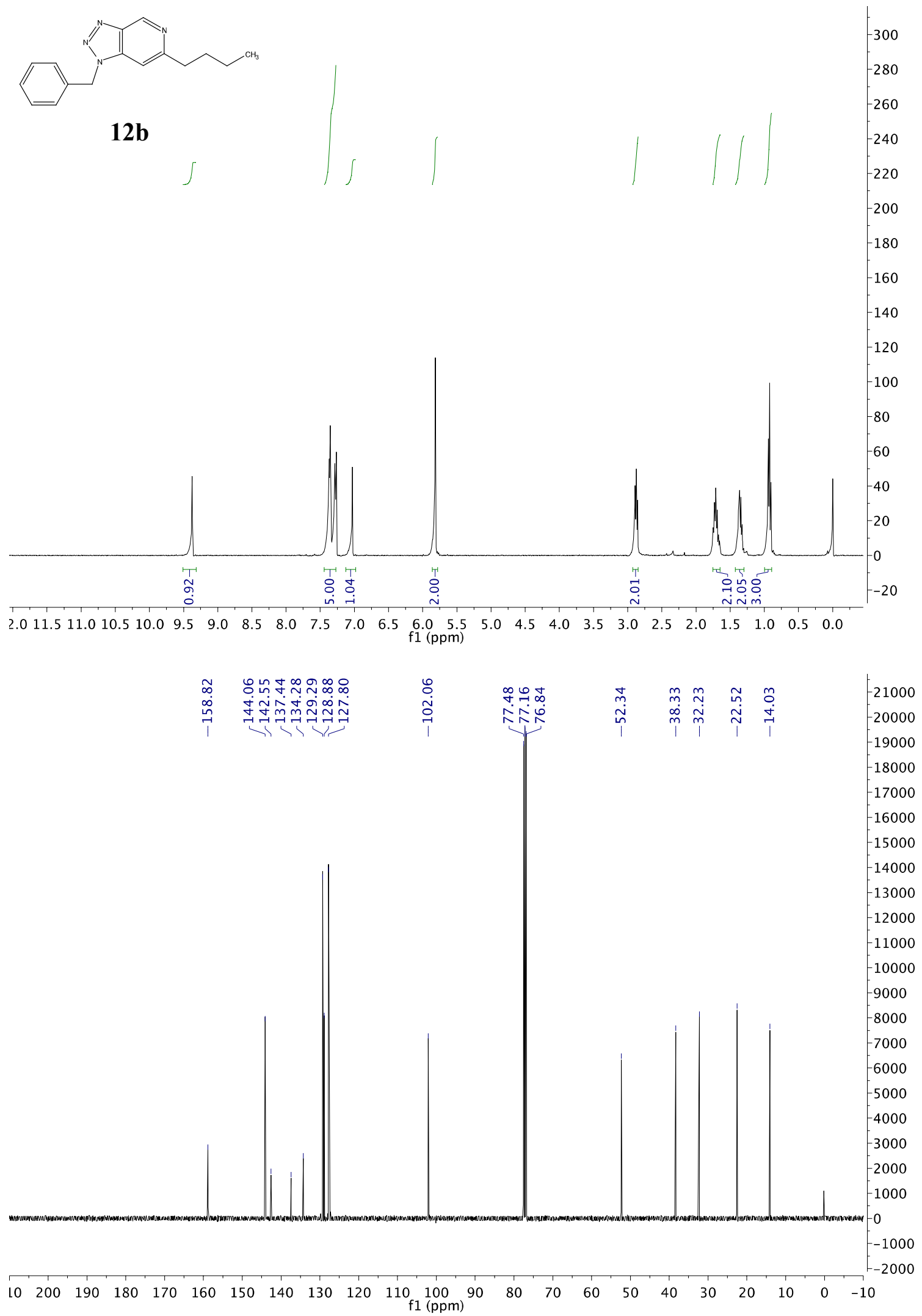

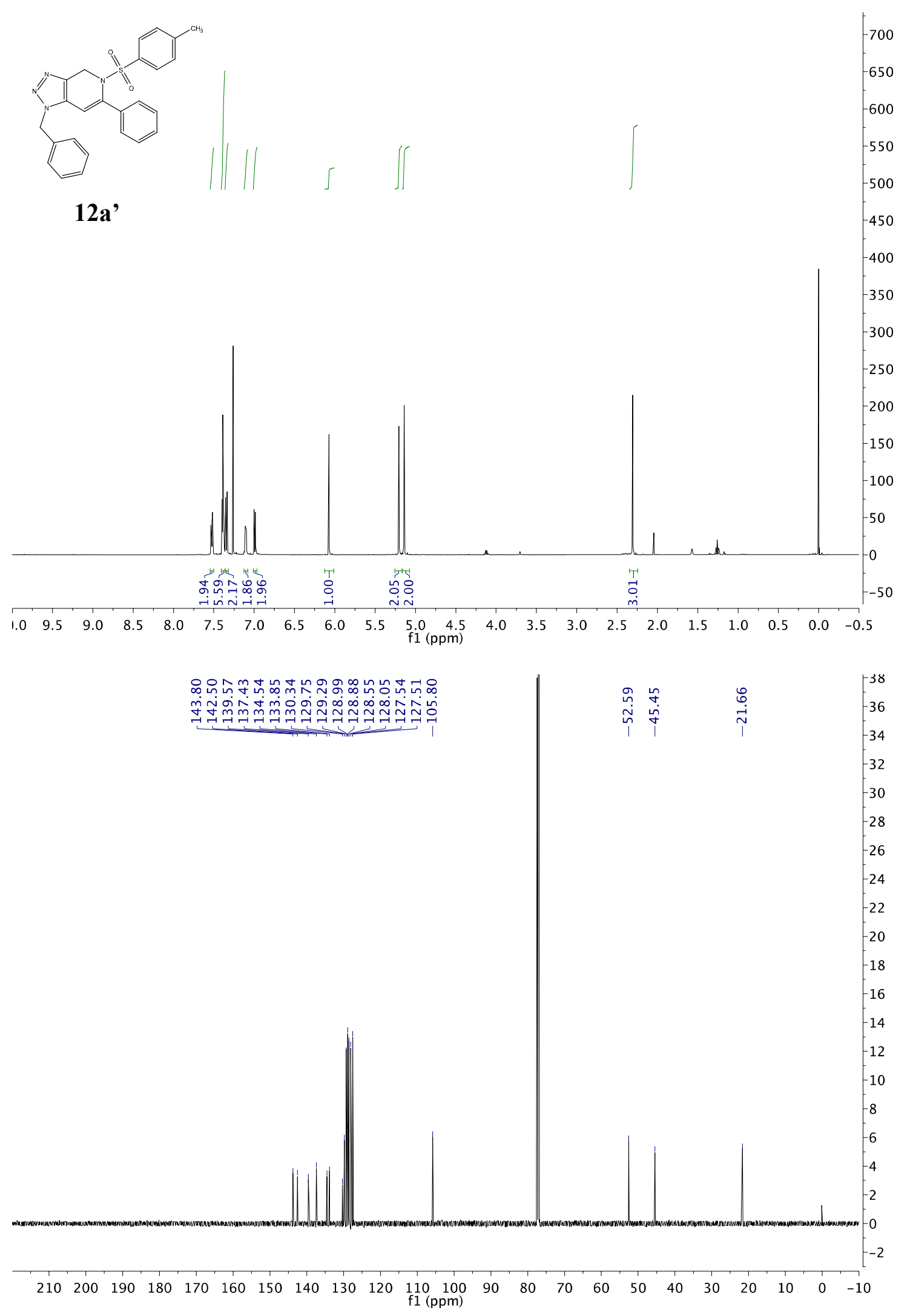

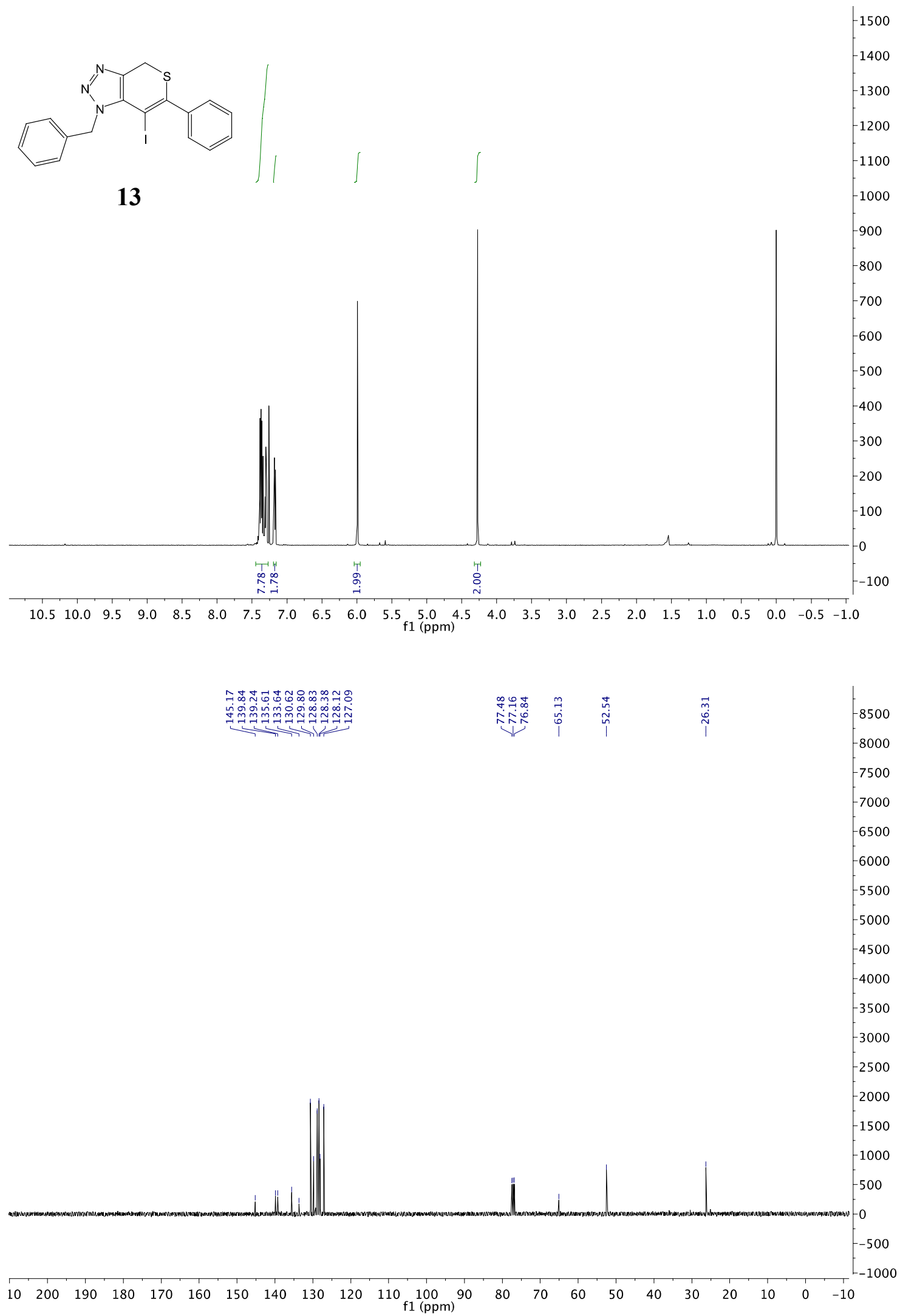